\title{
Influence of Weight Loss on Metabolic and Mitochondrial Function in the Mature Horse
}

Jennie L. Zambito

West Virginia University

Follow this and additional works at: https://researchrepository.wvu.edu/etd

\section{Recommended Citation}

Zambito, Jennie L., "Influence of Weight Loss on Metabolic and Mitochondrial Function in the Mature Horse" (2013). Graduate Theses, Dissertations, and Problem Reports. 393.

https://researchrepository.wvu.edu/etd/393

This Dissertation is protected by copyright and/or related rights. It has been brought to you by the The Research Repository @ WVU with permission from the rights-holder(s). You are free to use this Dissertation in any way that is permitted by the copyright and related rights legislation that applies to your use. For other uses you must obtain permission from the rights-holder(s) directly, unless additional rights are indicated by a Creative Commons license in the record and/ or on the work itself. This Dissertation has been accepted for inclusion in WVU Graduate Theses, Dissertations, and Problem Reports collection by an authorized administrator of The Research Repository @ WVU.

For more information, please contact researchrepository@mail.wvu.edu. 


\title{
Influence of Weight Loss on Metabolic and Mitochondrial Function in the Mature Horse
}

\author{
Jennie L. Zambito, M.S., PAS
}

\begin{abstract}
A Dissertation submitted to the Davis College of Agriculture, Natural Resource and Design at West Virginia University in partial fulfillment of the requirements for the degree of

\author{
Doctor of Philosophy \\ in \\ Agricultural Sciences
}

\author{
Kimberly M. Barnes, Ph.D., Chair \\ Janet C. Tou, Ph.D. \\ Stephen E. Alway, Ph.D., FACSM \\ John M. Hollander, Ph.D. \\ Holly S. Spooner, Ph.D., PAS
}

Department of Animal and Nutritional Sciences

Morgantown, West Virginia

2013

Keywords: Obesity, Horse, Weight loss, Mitochondria, Glucose, Lipid

Copyright 2013 Jennie L. Zambito 


\section{ABSTRACT \\ Alterations of Metabolic and Mitochondrial Function in Response to Weight Loss \\ Jennie L. Zambito}

Obesity causes a multitude of metabolic issues in the horse, yet stepwise alterations in glucose and lipid metabolism, mitochondrial capacity and oxidant status during weight loss have yet to be analyzed. Skeletal muscle contains subsarcolemmal (SSM) and interfibrillar (IFM) mitochondria, which respond differently to physiological stimuli, impacting tissue and whole body oxidant status. We hypothesized that 8 light-type horses would display improvements in skeletal muscle mitochondrial subpopulation function and decreases in circulating oxidant status markers during weight loss from an obese ( 7 to 8) to moderate (5) body condition score (BCS). Additionally, we hypothesized improvements in morphometric measurements, circulating metabolic markers, along with glucose tolerance and insulin sensitivity would also be seen. Change in BCS and percent weight loss were related to decreases in all morphometric measurements $(p<0.05)$ except for abdominal circumference $(\mathrm{p}<0.10)$. Measurements of body composition also decreased with regard to both weight loss parameters $(\mathrm{p} \leq 0.001)$. Circulating markers of lipid metabolism evaluated from non-fasting samples revealed no changes in nonesterified fatty acid (NEFA) or triacylglyceride concentration, yet ex vivo lipolysis media was decreased in response to weight loss $(\mathrm{p}=0.07)$ and BCS change $(\mathrm{p}=0.01)$. Insulin sensitivity calculated from baseline and post-weight loss intravenous glucose tolerance tests was improved after weight loss and corresponding BCS decline $(p \leq 0.05)$ while the disposition index, reflecting $\beta$-cell responsiveness, also increased due to weight loss $(\mathrm{p}=0.09)$. Plasma nitrate decreased in response to BCS reduction $(\mathrm{p}=0.06)$ and percent 
weight loss $(\mathrm{p}=0.06)$, whereas erythrocyte total glutathione $(\mathrm{p}=0.06)$ concentration increased with decreasing BCS, signifying improvements in oxidant status. Mitochondrial electron transport chain complex I and IV displayed greater activity in SSM than IFM $(\mathrm{p} \leq 0.05)$, while I, III, and IV in SSM had decreased activity due to BCS change and percent weight loss $(\mathrm{p} \leq 0.01)$. Interactions between SSM and IFM complex IV activity and both weight loss markers $(\mathrm{p}<0.05)$ were displayed. Citrate synthase activity, indicating mitochondrial number, was greater in SSM than IFM $(p<0.0001)$ but remained unchanged with weight loss parameters. Lipid peroxidation was decreased due to BCS change $(p=0.01)$ and weight loss $(p=0.02)$, with greater concentration in SSM, but a larger decrease in IFM $(\mathrm{p} \leq 0.05)$. Alterations in complex activities and lipid peroxidation suggest IFM are more affected by weight loss, with large contributions from complex IV byproducts. Mitochondrial component flexibility may contribute individually to development and disease propagation along with athletic performance of the equine athlete. Lack of changes in circulating markers along with minimal changes in minimal model parameters suggest that while horses were obese, metabolic function was conserved. Further evaluation of metabolically healthy but obese horses may provide insight as to management and treatment of obesity associated maladies. 
Alterations of Metabolic and Mitochondrial Function in Response to Weight Loss

Jennie L. Zambito

Dissertation submitted to the Davis College of Agriculture, Natural Resource and Design at West Virginia University in partial fulfillment of the requirements for the degree of

Doctor of Philosophy in Agricultural Sciences

Department of Animal and Nutritional Sciences

APPROVAL OF THE EXAMINING COMMITTEE

Janet C. Tou, Ph.D.

$\overline{\text { Stephen E. Alway, Ph.D., FACSM }}$

John M. Hollander, Ph.D.

Holly S. Spooner, Ph.D., PAS

DATE

$\overline{\text { Kimberly M. Barnes, Ph.D, Chair }}$ 


\section{ACKNOWLEDGEMENTS}

A special thanks is warranted for Dr. Holly Spooner. Thank you for taking a chance on me as your first graduate student so long ago; this experience has been better than any I could have imagined. I have learned so much from you in many different capacities and am honored to be able to call you not only a mentor but also a friend.

I would also like to thank Dr. Kim Barnes, who graciously accepted me as a student and assisted tremendously in the completion of this project. Her guidance, patience, leadership and inspiration provided a capstone to complete the final stages of my education at WVU.

I am incredibly fortunate to have a supportive, encouraging committee that aided in guiding and refining my skills. Thank you to Dr. Stephen Alway, Dr. John Hollander and Dr. Janet Tou for their confidence in my abilities and mentorship in scientific reasoning and logic. It was a privilege to work with them and I look forward to continued collaboration in the future.

Numerous people have contributed to the completion of this dissertation. Thank you to Dr. Rhonda Hoffman, Ellen Goertzen, Sarah Newton-Cromwell, E. Hannah Hoblitzell, and Morgan Bush, along with MTSU graduate students contributed greatly to many portions of this project including sample collection, data analysis and interpretation. I would like to especially recognize Cody Nichols for his contribution to this project and for his unfailing friendship. Many times of laughter, research, and gym time have been spent in his company and he is largely responsible for my sanity through most of this project. 
I would be sorely remiss without recognizing Scott \& Grace Spooner. Thank you so much for opening your home to me during the course of this project. Scott, your assistance through all the projects at WVU and MTSU both at the farm and behind the scenes is greatly appreciated. Also, I would like to thank Terri and Randy Wotring for their support, understanding and encouragement through the last couple years. You have contributed more than you realize to the completion of this dissertation.

The encouragement of all my friends and family has been motivation needed to propel me through this portion of my education. I am forever grateful to Alycia Kauffman, Katelyn Gentry, Vanessa Faljean, Louisa Bowen, Mike and Wanda King, Erin and Paige Carver and Meghan Manor. They kept me motivated and focused through my time at WVU. I am especially thankful for the love and support of my grandparents, Carolyn and Gil Fall.

From as far back as I can remember my parents, Ed and Linda Zambito, have been my biggest supporters, cheerleaders and confidants. They have been with me every step of the way, through good times and bad, shaping and molding me into the person I am today. Thank you so much for everything you have done for me, it has allowed us to make our dreams come true. I love you both so much and am so incredibly grateful to be your daughter. My sister, Allie Zambito, has also been a huge part of my life. She always knows the right thing to say to make me laugh and remain focused. Having her as my sister has made me work harder than I knew was possible.

Bill Ivey has played a critical role in the completion of my doctorate. Even from afar he has supported me in every facet of my education, from sample collection, job searches, trying moments, companionship, and everything in between. Thank you for 
always encouraging me to push on, never give up, and remembering to balance work and the things I enjoy most. Words cannot express how much your support means to me. I love you and am excited to see what lies ahead for us.

I would also like to recognize and dedicate this dissertation to my grandparents, Dr. Arthur and Kathryn Zambito, along with my parents, Ed and Linda Zambito, for their countless contribution to my education. Without them, achieving this dream would not be possible and I am forever grateful to all of you. Grandma and Grandpa - I thought of you often during this journey and I hope you are watching up there! 


\section{TABLE OF CONTENTS}

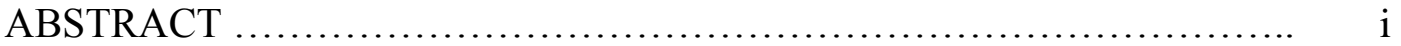

APPROVAL OF THE EXAMINING COMMITTEE ........................ iii

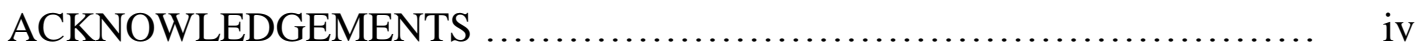

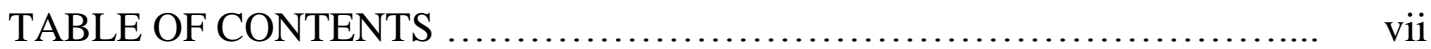

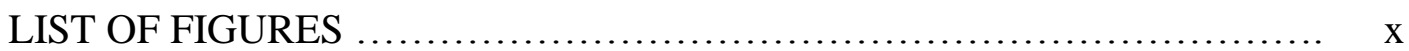

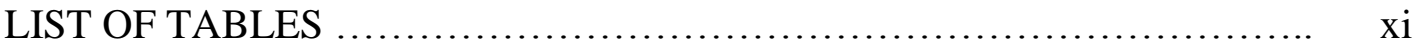

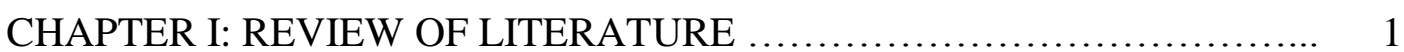

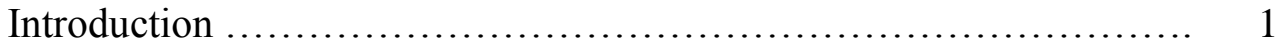

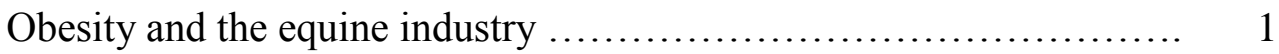

Normal metabolic function: nutrient intake and utilization in fed vs fasting states ................................................ 4

Glucose metabolism .................................... 4

Lipid metabolism ................................... 6

Mitochondrial metabolism ................................ 12

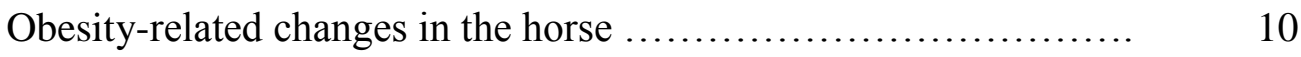

Thermoregulation $\ldots \ldots \ldots \ldots \ldots \ldots \ldots \ldots \ldots \ldots \ldots \ldots \ldots \ldots \ldots \ldots \ldots, \quad 10$

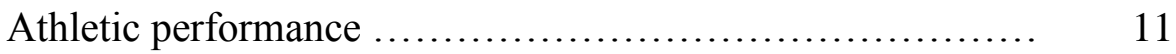

Reproductive performance $\ldots \ldots \ldots \ldots \ldots \ldots \ldots \ldots \ldots \ldots \ldots . . \ldots \ldots \ldots$

Metabolic impacts of obesity ................................. 13

Adipose tissue metabolism $\ldots \ldots \ldots \ldots \ldots \ldots \ldots \ldots \ldots \ldots \ldots \ldots . \ldots, 14$

Skeletal muscle glucose and lipid metabolism ............... $\quad 15$

Mitochondrial function and oxidative status ................ 17

Differences in body condition .................................. 19 
Effect of obese versus lean body condition on metabolic function

Figures.

CHAPTER II: JOURNAL ARTICLE I: Weight loss impacts on nutritional status and metabolic markers in a mature equine model

Abstract

Introduction 25

Materials and Methods

Results

Discussion

Conclusions

Tables and Figures

46

CHAPTER III: JOURNAL ARTICLE II: Changes in oxidant status and mitochondrial function in response to weight loss in an obese horse model

Abstract

Introduction

Materials and Methods

63

Results

Discussion

Conclusions

Tables and Figures

CHAPTER IV: LIMITATIONS

CHAPTER V: OVERALL IMPLICATONS, CONCLUSIONS, AND FUTURE DIRECTIONS 


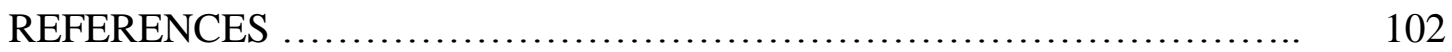

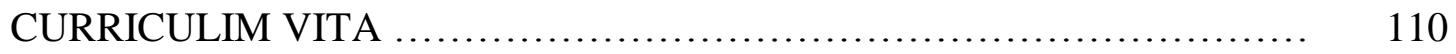

APPENDICES: RAW DATA ..................................... 113

Appendix A: Circulating Oxidant Status and Metabolic Markers ..... 113

Appendix B: Morphometric Measurements ..................... 115

Appendix C: Body Composition Measurements .................. 118

Appendix D: Frequently Sampled Intravenous Glucose Tolerance Test Data ............................................... 120

Appendix E: Lipolysis and Weight Loss Parameters ............... 128

Appendix F: Mitochondrial Complex Activities, Citrate Synthase and

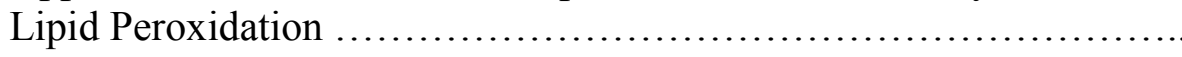




\section{LIST OF FIGURES}

Literature Review Figures

Figure 1: Entry of fatty acids into the mitochondria ................. 22

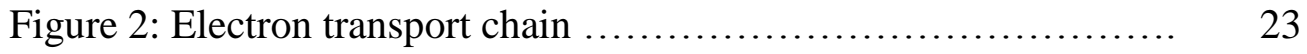

\section{Chapter II Figures}

Figure 1A-D: Effects of decreasing body condition and percent weight

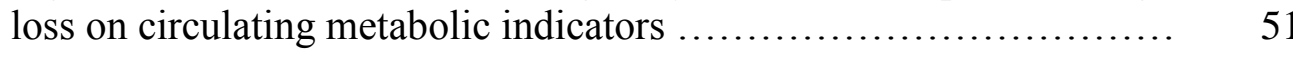

Figure 2A-B: Influence of weight loss parameters on lipolysis markers ... 53

Figure 3 A-B: Glucose and insulin curves during baseline and end weight loss frequently sampled intravenous glucose tolerance tests .......... 55

Figure 4 A-B: Representative illustrations of glucose and insulin curves during each frequently sampled intravenous glucose tolerance test by

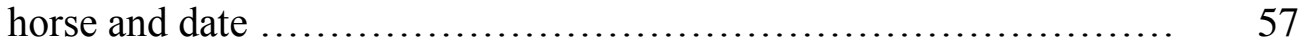

\section{Chapter III Figures}

Figure 1 A-C: Changes in circulating oxidant status markers in relationship to change in body condition score and weight los.................. 86

Figure 2 A-D: Mitochondrial subpopulation electron transport chain enzyme activities in response to change in body condition score or

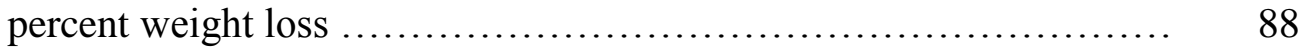

Figure 3: Mitochondrial subpopulation citrate synthase activity as an indicator of population size in response to change in body condition score or percent weight loss ......................................

Figure 4: Lipid peroxidation in mitochondrial subpopulations in response to change in body condition score or percent weight loss ..... 


\section{LIST OF TABLES}

Chapter II Tables

Table 1: Individual characteristics of study horses ................. 46

Table 2: Nutrient analysis of grass hay and concentrate, as fed ........ 47

Table 3: Percent composition of diet on a dry matter basis ............ 47

Table 4: Morphometric measurements during weight loss ............. 48

Table 5: Association of body fat measurements with body condition score

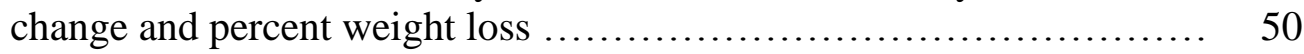

Table 6: Minimal model parameters during baseline sampling and end of weight loss period frequently sampled intravenous glucose tolerance tests.. 59

Table 7: Correlation of morphometric measurements, body composition and circulating metabolic markers $\ldots \ldots \ldots \ldots \ldots \ldots \ldots \ldots \ldots \ldots \ldots \ldots .60$

Chapter III Tables

Table 1: Individual characteristics of study horses ................ 83

Table 2: Nutrient analysis of grass hay and concentrate, as fed ........ 84

Table 3: Percent composition of diet on a dry matter basis ........... 84

Table 4: Physical descriptors of study horses during weight loss ...... 85

Table 5: Correlation of morphometric measurements, complex activity and circulating oxidant status markers 


\section{Chapter I: Literature Review}

\section{Introduction}

Obesity and its associated maladies plague both humans and horses in the United States today. Recent studies suggest nearly half the nation's horses may be overweight or obese, with 'easy-keeper' breeds at particularly high risk for unwanted weight gain (Geor et al., 2007; Henneke et al., 1983). A serious concern for horse owners and veterinarians, obesity is associated with equine metabolic syndrome and related conditions of insulin resistance, oxidative damage, chronic systemic inflammation, and laminitis (Geor et al., 2007). While knowledge of the effects of obesity on physiological pathways and metabolic parameters is growing, numerous areas still remain vague. Obesity is known to impact insulin sensitivity with confounding effects on glucose homeostasis, lipid metabolism, mitochondrial function and concomitant oxidative damage. Often, weight loss, as a result of caloric restriction and/or exercise, is touted to be the most beneficial means to decrease these effects of obesity; however, little is known about the impacts of obesity and the metabolic alterations occurring within an individual during stepwise decreases in body mass.

\section{Obesity and the equine industry}

Obesity affects a large population of the horses within the United States today. The prevalence of overweight and obese horses is much higher than initially estimated, with $41 \%$ of horses in the study found to be over conditioned or obese (Pleasant et al., 2008). By definition, obesity arises when energy intake exceeds energy expenditure, and this excess energy is primarily stored within the body as triglycerides or triacylglycerols 
(Bray, 2004). Genetics greatly influence predispositions for this phenotypic trait as displayed by breed differences, but other management practices such as diet and physical activity, along with developmental stage and age also modulate this condition (Kopelman, 2000). For the feral equine, the ability to store energy can be a vital means for survival when food sources are scarce, yet for the domestic horse where dietary intake is often not a concern, extended periods of obesity or cyclical fluctuations in weight gain and loss may cause numerous metabolic maladies.

Although some horses may possess an overweight body condition for an extended period of time, others may gain and lose weight through the course of the year, in conjunction with increased or decreased work load (e.g. show season vs. winter), or seasonal alterations in diet such as the availability of lush pasture. Pasture can often meet or exceed the nutritional needs of many types of horses, despite seasonal variation between growing and dormant stages, along with diurnal alterations in nutrient status of grasses and legumes (NRC, 2007). Since starch content is contained within the chloroplast, starch saturation is easily achieved in warm season grasses due to chloroplast limitations, yet fructans are typically stored within vegetative structures, and can therefore be present in much higher concentrations as seen in cool season grasses (Bender and Smith, 1973; Ojima and Isawa, 1968).

Understanding and managing forage and concentrate intake for horses of any breed, nutritional status, or workload, is necessary to maintain adequate health as obesity and over-nutrition due to improper management poses numerous health risks. Managing the 
obese horse can be challenging for many horse owners due to the multitude of problems that can arise regarding metabolic function. Weight loss is often the primary solution for the overweight horse; however, while much is known about the effects of obesity, little knowledge is available regarding the effects of weight loss after a bout of obesity. While the average horse owner is not concerned with the specifics of why obesity is harmful to the horse, knowledge of how obesity and subsequent weight loss affects metabolic parameters can educate those marketing nutraceuticals and pharmacological agents to the general public. In the obese horse, insulin insensitivity, hyperglycemia, and impaired lipid metabolism can give rise to dysfunction within the mitochondria and increased oxidative damage (Geor et al., 2007). In addition, further understanding of how the horse handles metabolic substrates on a cellular level can provide insight into fueling the entire body during times of excessive nutrients and caloric restriction, which can be important when managing both performance, and companion equines alike. Although a reduction in body condition to a moderate level is beneficial for the health of an animal or person, the malfunctions caused by obesity within glucose processing, lipid metabolism and mitochondrial function may have lasting effects. In order to gain a full appreciation for the implications of obesity on physiological function, a thorough understanding of the proper functions of these systems is warranted. 


\section{Normal metabolic function: nutrient intake and utilization in fed vs. fasting states}

\section{Glucose metabolism}

Hormonal regulation is central to maintaining blood glucose concentrations at a relatively constant level. Balance between insulin, glucagon and other hormones is necessary to maintain metabolic homeostasis. Normal equine blood glucose ranges $40-70 \mathrm{mg} / \mathrm{dL}$ (Hoffman et al., 2003); inability to maintain this narrow range results in a multitude of detrimental changes to overall homeostasis within the body. For example, in horses prolonged elevation of glucose concentrations has a toxic effect on cells (Yki-Jarven, 1992), whereas low concentrations can produce seizures and disorientation (Valberg and Firshman, 2009). The effects of these hormones reach far beyond their role in glucose metabolism and many intermediates within these pathways feed into other catabolic and anabolic states. Prolonged periods of fed or fasting metabolism can cause obesity and insulin resistance, or starvation, respectively. Achievement of optimal body condition, performance and physiologic mechanisms is possible with proper management and understanding of the detailed hormone functions within these systems.

Circulating glucose can face multiple fates, including entry into the adipose tissue, skeletal muscle and liver. Glucose movement into the liver occurs through passive diffusion, where as uptake into adipose and skeletal muscle tissue is insulin dependent. Upon elevation of blood glucose concentration, glucose is transported into the pancreatic $\beta$-cell via facilitated diffusion though GLUT 2, an insulin independent transporter. Elevated blood glucose concentration is directly reflected in within the pancreatic $\beta$-cell and thus will elicit an appropriately large insulin response (Straub and Sharp, 2002). 
Membrane depolarization occurs in response to glucose entry, leading to intracellular calcium release, which initiates a signaling cascade for the release of insulin-containing secretory vesicles via exocytosis (Straub and Sharp, 2002). Insulin is then able to reach skeletal muscle and adipose tissue via circulation and elicit glucose uptake to reduce blood glucose concentrations.

When insulin reaches the target tissue, binding of the hormone to the cell-surface insulin receptor induces autophosphorylation at a number of tyrosine residues on the receptor. Phosphorylation of specific residues allow for recognition by insulin receptor substrates (IRS), which are recruited to the receptor at the time of insulin binding and serve as docking sites. The insulin receptor then phosphorylates IRS at multiple tyrosine residues, allowing for recognition by the p85 regulatory subunit of phosphatidyl-inositol 3-kinase (PI3-kinase). Upon this activation, the p110 catalytic subunit of PI 3-kinase can then phosphorylate and convert phosphatidylinositol-2-phopshate (PIP2) to phosphatidylinositol-3-phosphate (PIP3) (Watson and Pessin, 2001). Protein kinase B is then activated by PIP3, which functions to recruit GLUT 4 containing vesicles to the plasma membrane. Facilitated diffusion of glucose into the cell can then occur, aiding in the decrease of blood glucose concentration. Translocation of GLUT 4 to the plasma membrane is primarily driven by insulin, and is thus considered an insulin dependent transporter (Goodyear and Kahn, 1998). Receptor sensitivity and effectiveness of the aforementioned signaling pathway is necessary to facilitate this response; any impairment can result in hyperglycemia and propagation of other associated maladies. 


\section{Lipid metabolism}

Fatty acid (FA) metabolism is closely intertwined with glucose metabolism within the skeletal muscle and adipocyte and therefore must be considered when evaluating the effects of obesity on metabolic parameters. Fatty acid metabolism within the body involves a delicate balance between multiple steps including lipolysis and long chain fatty acid release from adipocytes, delivery of dietary or released fatty acids to muscle cells via transport across the plasma membrane, lipolysis of intramuscular triglyceride stores, activation of the fatty acid and transport into the mitochondria for oxidation (Holloway et al., 2009). In order to gain a big picture understanding of how fatty acid release from the adipose tissue and utilization of the substrate within the muscle is interrelated with obesity and weight loss consideration must be given to both adipose and muscle tissues, including individual mitochondrial subpopulations within the muscle. Further, to fully understand changes associated with excess lipid stores in the body, familiarity with lipid metabolism in the tissues and their contribution to energy production and intracellular signaling is necessary.

A typical ration of dietary forage and concentrate components would yield a variety of triacylglycerols, phospholipids and cholesterols, each contributing individually to the lipid profile of the horse's diet. De novo fatty acid synthesis occurs during times of energy excess, in the cytosol of liver and adipose tissue cells. In this biosynthetic pathway, condensation reactions are coupled with the hydrolysis of ATP causing the reaction to be driven to completion; ultimately leading to excess storage of fats in the adipose tissue. 
In a fasted or other catabolic state such as exercise, chylomicron levels decline, and the adipose tissue becomes the major source of fatty acids for the body (Muoio and Newgard, 2006). A lower insulin-to-glucagon ratio, along with increased catecholamine levels due to a fasting/catabolic state, cause activation of hormone sensitive lipase (HSL). During lipolysis in the adipose tissue, HSL acts on diacylglycerols to release free fatty acids and glycerol into the circulation for use by other tissues. Concurrently, signaling within the body decreases glucose utilization while increasing fatty acid oxidation. Cytosolic citrate is used for synthesis of acetyl-CoA and malonyl-CoA, yet when acetyl-CoA carboxylase is phosphorylated and thus inactivated in the presence of AMP kinase, conversion of acetyl-CoA to malonyl-CoA ceases and concentrations decline. Low levels of malonylCoA combined with the effects of decreased citrate concentrations result in suppression of de novo lipogenesis and simulation of fatty acid oxidation through removal of malonyl-CoA inhibition of carnitine palmitoyltransferase-1 (CPT-1) activity. Elevated CPT-1 activity can ultimately lead to increased flux through fatty acid $\beta$-oxidation, as displayed in figure 1. Lastly, during times of increased fatty acid oxidation accumulation of acetyl-CoA, NADH and ATP allosterically inhibit pyruvate dehydrogenase. Overall, the actions of the mechanisms described during the fasting/catabolic state function to increase the rate of fat oxidation while decreasing glucose oxidation.

\section{Mitochondrial metabolism}

Mitochondria are double-membrane organelles specialized to convert energy dense macronutrients into ATP through oxidative phosphorylation, and also play a role in 
various cellular signaling pathways including apoptosis, and cellular proliferation (Martinez, 2006). The production of ATP is achieved through oxidation of acetyl-CoA from two major sources: from the end products of glycolysis, which enter the mitochondrion as pyruvate and are converted to acetyl-CoA via pyruvate dehydrogenase, and fatty acid oxidation end products. The citric acid cycle then utilizes acetyl-CoA to form citrate from oxaloacetate by the action of citrate synthase, and is rate limited by the action of isocitrate dehydrogenase converting isocitrate into $\alpha$-ketoglutarate. For each acetyl-CoA to enter the cycle, three $\mathrm{NADH}^{+}$, a FADH 2 and an ATP are generated. The $\mathrm{NADH}^{+}$and $\mathrm{FADH}_{2}$ are then able to carry electrons, or reducing equivalents, to the electron transport chain, where incremental releases of energy are applied to pump protons across the inner mitochondrial membrane. This is accomplished through the action of four major enzymes embedded within the inner mitochondrial membrane; NADH dehydrogenase (Complex I), succinate dehydrogenase (Complex II), cytochrome bc1 complex (Complex III) and cytochrome c oxidase (Complex IV, figure 2).

Alterations in mitochondrial number are often needed to manage ever-changing energy substrate loads and metabolic demands placed on various tissues. Skeletal muscle mitochondrial biogenesis can be stimulated through the actions of peroxisome proliferator-activated receptor gamma (PPAR) coactivator1-alpha (PGC-1 $\alpha$ ) (Scarpulla, 2008). In its active form, PGC-1 $\alpha$ interacts with nuclear receptor PPARs to increase gene transcription, along with the potential for PPARs to act in a feed forward fashion and increase PGC-1 $\alpha$ further (Muoio and Newgard, 2006). It is through this interaction with PPAR that PGC-1 $\alpha$ is able to increase mitochondrial biogenesis via increased 
transcription of nuclear respiratory factors (NRFs), along with influencing oxidative phosphorylation gene regulation (Lin et al., 2002). Mitochondrial autophagy, or mitophagy, can also occur in response to cellular environmental factors or stressors. Utilization of this pathway can be activated in response to overwhelming oxidative damage, mitochondrial dysfunction or damage to critical proteins used for mitochondrial function (Lemasters, 2005). Mitochondrial number, whether in response to biogenesis or autophagy, can be indicative of overall aptitude of the tissue to respond to physiological stimuli along with providing a detailed image of disease states and energy production.

While mitochondria are needed in all cell types to provide ATP, skeletal muscle contains two sets of biochemically and spatially distinct mitochondria: subsarcolemmal mitochondria (SSM), which reside beneath the plasma membrane, and interfibrillar mitochondria (IFM) which are located between the myofibrils (Palmer et al., 1977). While they function to achieve the same general goal, differences have been found in their morphology, biochemical properties and response to physiological stimuli, including exercise and stress (Cogswell et al., 1993; Manneschi and Federico, 1995; Palmer et al., 1985). Subsarcolemmal mitochondria are located just beneath the sarcolemma and are larger, with a more lamellar shape, while IFM tend to be smaller, more compact and located between the contractile filaments (Koves et al., 2005). In response to exercise, SSM tend to display greater increases in volume, state III respiration and enzymatic activity than IFM (Bizeau et al., 1998; Kreiger, 1980), and during times of muscle disuse SSM show more pronounced decrements (Bizeau et al., 1998). Further, oxidative phosphorylation properties with respect to mitochondrial 
subpopulation are different based on fiber type, and are individually affected in response to energy stress (Kim et al., 2002; Koves et al., 2005). Understanding the relationship between SSM and IFM and their contributions to skeletal muscle metabolism can be instrumental in understanding the alterations caused by increases or decreases in body condition, and therefore will be the focus of latter portions of this review.

\section{Obesity-Related Changes in the Horse}

\section{Thermoregulation}

While over-conditioned animals are suspected to be at higher risk for metabolic dysfunction, excess body fat may be advantageous to horses in cold climates. Body fat provides insulating properties along with providing an available energy source during times of low ambient temperatures. Previous studies have determined horses with a BCS of 7-9 require less supplemental feed during cold weather than horses with lower BCS (Cymbaluk and Christison, 1990). Conversely, this same property most likely induces heat stress in obese horses during hot/humid weather (Cymbaluk and Christison, 1990; Webb et al., 1989). Horses with a BCS of 7.5 or greater had a more difficult time dissipating heat in an ambient temperature of $31.5^{\circ} \mathrm{C}$ (humidity 43.8\%) than horses with an average BCS of 5.2 (Webb et al., 1989). Further, these obese horses had a higher respiration rate following a light intensity exercise bout consisting of 30 min of mixed walk, trot and canter work. While thermoregulation is crucial to homeostatic maintenance of the horse, performance from both an athletic and reproductive standpoint is of great interest to the industry and recreational horse owners alike. 


\section{Athletic Performance}

Currently within the equine industry, the impact of obesity on exercise performance has not been studied in depth. Preliminary research in this area showed a negative relationship between body weight and body fat percentage in performance measures of equine athletes (Thornton et al., 1987). In a study evaluating body composition of standardbred horses competing in one-mile races, larger amounts of body fat determined by rump fat ultrasound were negatively correlated to running performance, indicated by race times (Kearns et al., 2006b). Additionally, horses completing a $151 \mathrm{~km}$ endurance race were found to have lower BCS and carried less body fat $(26 \mathrm{~kg})$ than non finishers (45kg) (Lawrence et al., 1992).

Despite this valuable information, the previously mentioned studies were completed on horses with an average BCS of 4.5 and body fat percentage of $8.65 \%$ (Kearns et al., 2006b; Lawrence et al., 1992; Webb et al., 1989). It remains likely that horses with higher BCS would display further performance impairments compared to lean counterparts, especially if not enrolled in an exercise program.

\section{Reproductive Performance}

Body condition score to either an excessively high or low condition appears to impact estrous cycle length in broodmares, yet ovulation and fertility remain relatively unaffected with obesity. In mares with a BCS ranging from 7.5-8.5 continued to cycle during winter months, forgoing seasonal anestrus typically displayed by moderate and lean horses (Gentry et al., 2002). Higher circulating levels of leptin have been found in 
obese mares $(8.27 \pm 1.02 \mathrm{ng} / \mathrm{mL})$ compared to lean mares $(\sim 2 \mathrm{ng} / \mathrm{mL})$, potentially contributing to the absence of an anestrous period in this cohort (McManus and Fitzgerald, 2003). This concept is further supported as when leptin levels declined, obese mares entered into an anestrous period. Cause of the decline during this study remains unknown, as it was not accompanied by a reduction of body fat percentage, but may have been affected by fluctuations in overall satiety (McManus and Fitzgerald, 2003). Conversely, other studies suggest excessive body fat in mares $(\mathrm{BCS}>7)$ may prolong luteal phase length and interovulatory intervals when compared to thin mares (BCS 4) (Sessions et al., 2004). Elevated levels of leptin and insulin observed in the fatter mares, combined with the presence of insulin resistance were though to have caused the observed extensions (Sessions et al., 2004). Insulin resistance combined with other physiological indicators may be the influential factor between mares retaining normal reproductive cyclicity and the onset of dysfunction.

Guidelines for mares in gestation and lactation suggest a proper BCS before, during and after pregnancy should allow mares to be sufficiently fat so as to prevent loss in condition due to fetal development, but not so overweight as to induce metabolic stress (Quinn et al., 2006). Mares fed to either gain weight or maintain a BCS of 6.6-7.1 during gestation and lactation periods had higher successful pregnancy rates, decreased number of estrous cycles per conception compared to mares allowed to lose weight, with a BCS ranging from 6.1-3.7 (Henneke et al., 1984). Further, low levels of body fat (BCS <5) resulted in reduced pregnancy rates when compared to mares of increasing body condition (5-8), as mares with a higher BCS displayed decreased times to first estrous and first ovulation, 
indicating improved reproductive efficiency (Henneke et al., 1984). Effects of obesity on reproductive capability in the stallion have not been fully evaluated. Obese stallions may have reduced sexual performance, potentially due more to the effects of obesity on athleticism and thermoregulatory properties over the impact of obesity on sperm production (Quinn et al., 2006). Influences of obesity on both mare and gelding metabolic function is often of a more pressing concern, as if the horse is able to remain in a healthy homeostatic state, then reproductive function will also be conserved.

\section{Metabolic Impacts of Obesity}

Obesity is known to cause alterations in transport and metabolism of various energy substrates, yet exact mechanisms for obesity-associated disturbances remain elusive. Obesity-induced insulin resistance can be linked to many pathologic distrubances within the skeletal muscle, ultimately resulting in disruptions in oxidative and storage pathways for glucose and lipid molecules. Insulin resistance by definition is a condition of low insulin sensitivity in which the ability of insulin to lower circulating glucose levels is impaired (Henry, 2003). Originally, insulin resistance was thought to arise from extended periods of hyperglycemia, and subsequent insulin release from the pancreatic beta cells. Elevated levels of both glucose and insulin over time cause receptor insensitivity to normal levels and as the cycle propagates the condition worsens. It is now known that multiple factors affect insulin resistance, including this pathway and others arising from disconcerted metabolic communication between tissues and cellular compartments. 


\section{Adipose tissue metabolism}

Partitioning of lipids between adipose tissue and other peripheral tissues is integral in coordinating energy balance and signaling pathways regarding substrate utilization. As adipose tissue is designed to store triacylglycerides, enhanced lipolysis and free fatty acid flux from adipocytes exposes skeletal muscle and other tissues to a substantial fatty acid load, resulting in lipid accumulation in these tissues (Horowitz and Klein, 2000). Mircoarray experiments on obese leptin-deficient mice indicate genes encoding for transcription factors involved in lipogenesis, including sterol regulatory-element binding protein-1c (SREBP-1c) and PPAR- $\gamma$, are down-regulated in adipose tissue, yet are upregulated in the liver (Nadler et al., 2000; Soukas et al., 2000). While PPAR- $\gamma$ is not typically expressed at high levels in the liver (Nadler et al., 2000; Soukas et al., 2000), induction of transcription is caused by elevated circulating levels of triacylglycerides, suggesting relative expression is indicative of fuel repartitioning during times of obesity (Attie and Scherer, 2009). Moreover, adipose tissue expansion needed to accommodate excess nutrients places demands on vascularization and remodeling pathways. If these pathways lag behind the need for increased triacylglyceride storage, hypoxic conditions may emerge, leading to increased inflammation via macrophage recruitment (Attie and Scherer, 2009). Mitochondrial dysfunction, such as enzyme impairment or electron transport chain deficiency, in the adipocyte during times of hyperglycemia can also contribute to impairment of energy balance, as a decrease in respiratory capacity can reduce GLUT 4 translocation along with increasing reactive oxygen species production, further propagating local and systemic inflammation. (Lin et al., 2005; Shi et al., 2008). 


\section{Skeletal muscle glucose and lipid metabolism}

The interplay of lipid metabolism with other energy substrates and signaling pathways is disconcerted in animals consuming diets in extreme excess of their current needs, or of those in an obese body condition. It has been well documented that obesity causes impaired insulin signaling and glucose tolerance (Hoffman et al., 2003; Kronfeld, 2005), manifested by decreased insulin-stimulated glucose transport and metabolism in skeletal muscle and adipocytes (Reaven, 1995) and increased levels of circulating free fatty acids (Kahn and Flier, 2000). In combination with decreased GLUT4 levels or translocation (Kahn and Flier, 2000) seen with an obese phenotype, dysfunction in the insulin signaling pathway is further propagated.

With the onset of obesity, triacylglycerides are not only stored in the adipose tissue, but begin to accumulate at unhealthy levels in the skeletal muscle, liver, kidney and heart (Muoio and Newgard, 2006) due to increases in circulating triacylglycerides and free fatty acids. Increased levels of fatty acid binding protein on the plasma membrane or fatty acid translocase/CD36 on the plasma membrane but not the outer mitochondrial membrane (Bonen et al., 2004) may play a critical role in increased fatty acid uptake, accumulation of intramuscular lipids and impaired insulin signaling (Consitt et al., 2009). Increases in intramuscular triglyceride stores, which, in addition to impairing GLUT4 translocation, may also propagate the development of insulin resistance (Montell et al., 2001). Further, long chain fatty acyl-CoAs are shunted away from CPT-1 and are instead are preferentially partitioned toward synthesis of signaling intermediates such as diacylglycerols and ceramide. Accumulation of these molecules combined with the 
effects of excess intramuscular lipid stores are thought to activate serine-kinases (Holland et al., 2007; Yu et al., 2002), along with impacting protein kinase c and other downstream insulin signaling molecules (Schmitz-Peiffer, 2002), thereby preventing proper translocation of GLUT4 in an insulin-dependent manner. Without the necessary actions of this transporter, the insulin mechanism fails to reduce circulating glucose concentrations and, therefore, contributes to obesity-induced insulin resistance.

An imbalance between fatty acid uptake and fatty acid oxidation in obese individuals is often seen, contributing to the aforementioned impairment in glucose tolerance along with decrements in regulatory steps of fatty acid oxidation. Reduced activity of carnitine palmitoyltransferase-1 (CPT-1), $\beta$-hydroxy acyl-CoA dehydrogenase, citrate synthase and cytochrome c oxidase have been reported in samples of skeletal muscle from obese subjects (Simoneau et al., 1999), along with overall decreased mitochondrial content (Kim et al., 2000). Overexpression of CPT-1 in L6 cells caused an increase in fatty acid oxidation and protected cells against fatty acid induced insulin resistance (Muoio and Newgard, 2006), suggesting that increasing $\beta$-oxidation could help to correct maladies driven by this accumulation. Obese individuals have been reported to have an increased ratio of glycolytic to oxidative enzyme capacity, as determined by hexokinase to citrate synthase activity levels, yet not as elevated as the ratios seen in type 2 diabetic subjects (Simoneau and Kelley, 1997). Further, this ratio was negatively correlated with insulin sensitivity, providing increasing evidence that an alteration in metabolic preference may contribute to insulin resistance seen in an obese individual. 


\section{Mitochondrial function and oxidative status}

While mitochondrial oxidative phosphorylation is a highly efficient process, a small amount of electrons can prematurely bind with oxygen to form free radicals. Further, under certain conditions such as obesity, excessive production of reactive oxygen species has been found and attributed to the mechanisms implicated in insulin resistance and metabolic syndrome (Martinez, 2006). A low degree pro-inflammatory state has also been associated with obesity, causing impairments in the oxidative stress and antioxidant mechanisms leading to activation of nuclear factor kappa- $\beta$ in the presence of excessive reactive oxygen species (Martinez, 2006). Additionally, reactive oxygen species have been attributed to propagating insulin resistance and paracrine communication between adipocytes and macrophages, thereby mediating inflammatory changes by means of free fatty acids and tumor necrosis factor- $\alpha$ (Valerio et al., 2006).

Accumulation of intramuscular lipid may place additional strain on the mitochondria and create a larger disconnect between metabolic pathway fluidity. For example, a metabolic profiling study demonstrated that obesity-induced rates of $\beta$-oxidation overwhelmed the citric acid cycle, resulting in incomplete fatty acid degradation (Koves et al., 2008). Lack of cycle completion caused accumulation of mitochondrial-derived by-products, including acylcarnitines, which potentially can contribute to obesity induced insulin resistance. Moreover, electron transport chain activity in the vastus lateralis muscle, assessed from complex activities normalized to creatine kinase activity, in type II diabetic and obese human subjects was found to be diminished when compared to that of healthy lean counterparts, even when corrected for total mitochondrial content (Ritov et al., 
2005). Although the exact mechanism linking mitochondrial capacity and insulin resistance propagation is unknown, these findings suggest a link between insufficient coupling and/or reduced enzymatic activity in insulin resistant obese individuals.

As previously mentioned, skeletal muscle contains different populations of mitochondria which respond differently to physiological stimuli. In response to hyperglycemia and hyperlipidemia situations changes in individual size, population size, proteins involved in substrate utilization, electron transport chain (ETC) function, and reactive species production have been observed in rodents (Dabkowski et al., 2010; Dabkowski et al., 2008a). Typically, in an obese or type II diabetic model, the SSM tends to be more affected, while in a type I diabetic model, the IFM display greater dysfunction (Dabkowski et al., 2010; Williamson et al., 2009). The hyperglycemic environment caused in response to obesity and metabolic syndrome leads to enhanced reactive oxygen species (ROS) production, which can facilitate enhanced apoptosis along with causing oxidative damage to the cell and its contents (Williamson et al., 2009). Further, reduced activity of citrate synthase and CPT-1 in obese skeletal muscle has been used to infer reduced mitochondrial number (Kim et al., 2000), a concept confirmed by lower mitochondrial DNA in obese human individuals compared to lean counterparts (Ritov et al., 2005).

Despite contributions from other sources, the mitochondrion is regarded as the primary site of production of ROS (Kelley et al., 2002b). Several locations within the ETC are prone to formation of ROS, and due to the inclusion of iron-sulphur centers, which can 
react with other ROS to produce the hydroxyl radical, can cause mitochondrial dysfunction (Fridovich, 1995). Dysfunctional mitochondria can result in further insult to the inner mitochondrial membrane, producing oxidative damage to lipids and proteins, which in turn limits adequate production of ATP. The effects of functional changes in mitochondria extend to fatty acid metabolism as well. In age-matched lean (BMI $23.3 \pm$ 0.7) and obese (BMI $37.6 \pm 2.2$ ) humans, citrate synthase, a common marker of total muscle mitochondrial volume and capacity for fatty acid oxidation was decreased in obese individuals (Holloway et al., 2007). Due to the role of the mitochondria within metabolism and generation of ROS and implications of cellular apoptosis with corresponding dysfunction, subpopulations of mitochondria may be differentially affected by physiological parameters presented with obesity and subsequent weight loss. Inability for mitochondrial function to return to a normal functioning state could be detrimental for the animal, as prolonged periods of increased oxidative damage could lead to further disease and impairment in the future.

\section{Differences in body condition}

Effects of obese vs. lean body condition on metabolic function

As the skeletal muscle has the ability to transition easily between oxidative and glycolytic metabolism, this tissue adapts to a given set of metabolic cues, thus having the largest differences between obese and lean phenotypes. This malleable characteristic may allow skeletal muscle mitochondria to rebound after prolonged periods in an obese state. In a 16-week lifestyle modification program, obese non-diabetic volunteers were placed on a calorie-reduced diet and a moderate-intensity exercise program (Menkishova et al., 
2006). Significant improvements were noted with regard to insulin sensitivity, whole body fat oxidation and electron transport chain oxidative capacity. Despite this increase, mitochondrial number, as measured by citrate synthase activity and mitochondrial DNA, quantification was not altered.

It has been well documented that weight loss improves insulin sensitivity; however, little is known about the impact of weight loss on skeletal muscle mitochondria, potentially due to the recent development in knowledge on the effects of obesity on mitochondrial functions (Toledo and Goodpaster, 2013). Current research in this area appears to be confounding with respect to improving mitochondrial capacity. In a 1 yr weight loss study of obese women who lost an average of $55 \mathrm{~kg}$, fatty acid oxidation was unchanged, along with mRNA content of CPT 1 and PGC- $1 \alpha$ indicating mitochondrial biogenesis is not active during periods of weight loss (Berggren et al., 2008). Yet, in a study of obese women who had undergone moderate weight loss by caloric restriction and light exercise, succinate dehydrogenase activity was increased (Kern et al., 1999). Therefore, it is currently unclear if changes in mitochondrial function associated with weight loss are due primarily to alterations in metabolic function, or are manifested as a side effect of contractile activity during exercise.

Evaluation of markers of metabolism and oxidative damage through a weight loss period can provide a stepwise analysis of changes seen from an overall body perspective to a cellular level of function. Knowledge to be gained from this study has implications spanning multiple facets, as information on cellular changes are important to determine 
how substrates are being handled on a chemical level, while implications of cellular products on body function is needed to elucidate the changes seen with regard to an increase and decrease in body condition. Within the equine industry, substrate utilization is needed for optimal performance along with maintenance, meaning the impact of data to be gained from this study has the ability to reach the performance industry and small farm practices alike. By developing a baseline understanding of the changes associated with weight loss on metabolic function, we can further evaluate the role of nutraceutical and pharmaceutical interventions designed to improve or adjust these parameters.

As of now, the majority of studies completed evaluating these parameters have been conducted either using two groups of animals: a lean and an obese group, or evaluating snapshot samples of pre- and post-weight loss. While this information is important in elucidating the damaging effects of obesity, little is known about how these parameters will change within an obese individual as body condition decreases over time. 


\section{FIGURES}

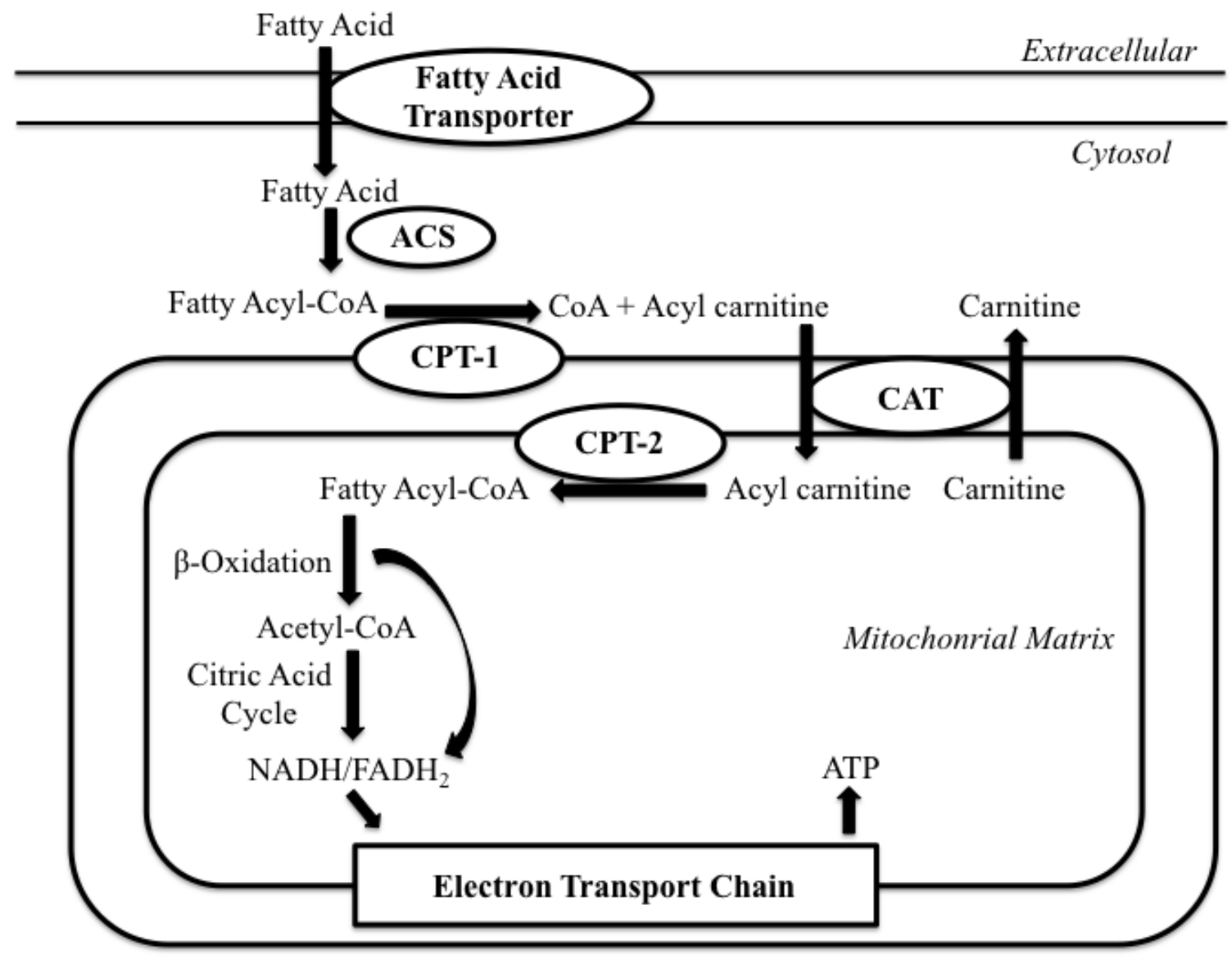

Figure 1: Entry of fatty acids into the mitochondria

ACS: Acyl-CoA synthetase, CPT-1: carnitine palmitoyltransferase-1, CPT-2: carnitine palmitoyltransferase-2, CAT: carnitine acyltransferase-1 


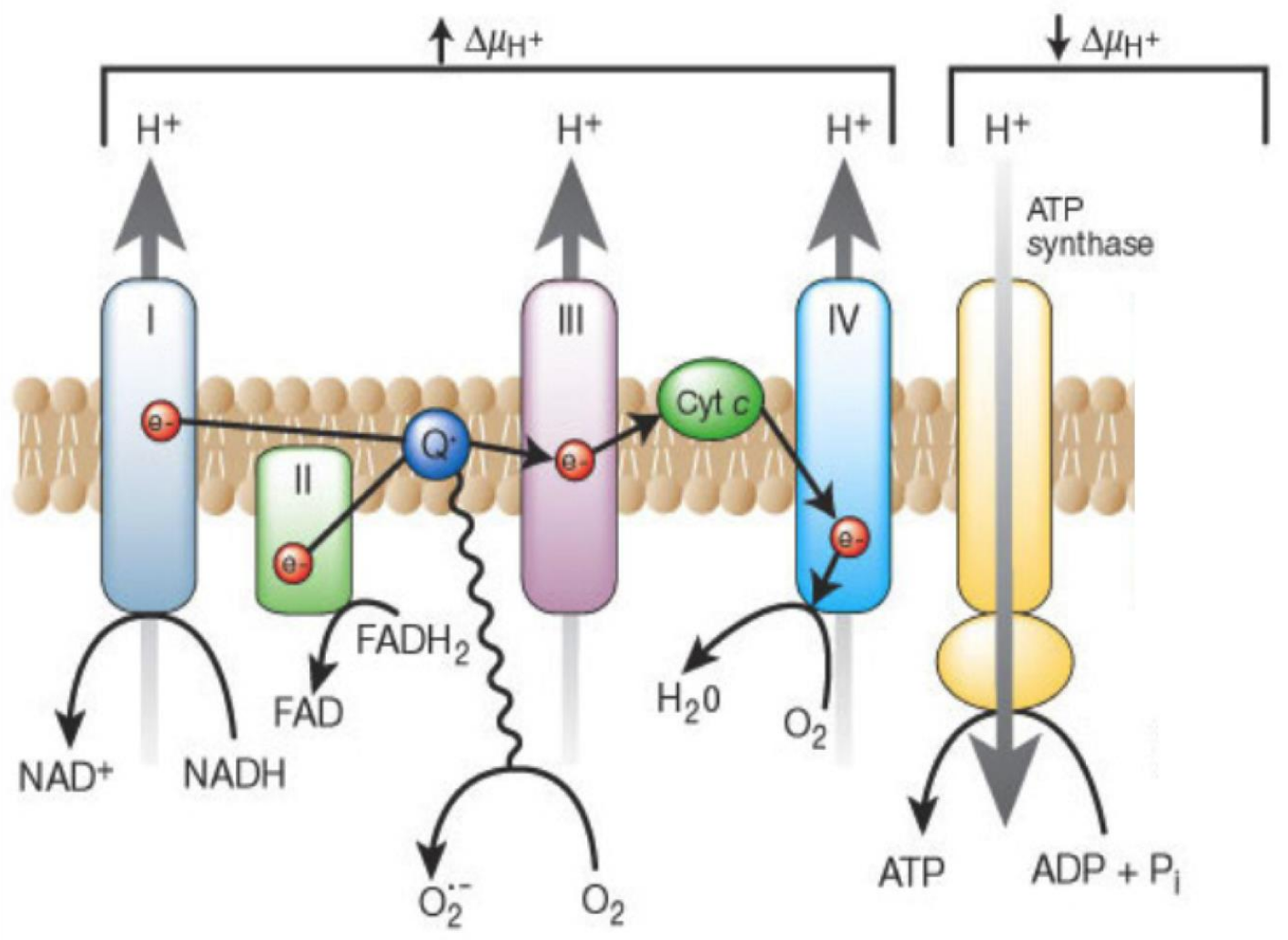

Figure 2: Electron Transport Chain Adapted from (Brownlee, 2001)

Q•: Ubiquinol, e-: electron, Cyt c: cytochrome c, $\Delta \mu \mathrm{H}+$ : change in proton gradient 


\title{
CHAPTER II: JOURNAL ARTICLE I \\ WEIGHT LOSS INFLUENCE ON NUTRITIONAL STATUS AND METABOLIC \\ MARKERS IN A MATURE EQUINE MODEL
}

\begin{abstract}
Obesity is known to impact insulin sensitivity with confounding effects on glucose homeostasis, lipid metabolism, mitochondrial function and concomitant oxidative damage. Often, weight loss as a result of caloric restriction and/or exercise is touted as beneficial in decreasing these adverse effects of obesity; however, little is known regarding lasting effects of obesity on metabolic parameters after weight loss has occurred. We hypothesized that 8 light-type horses would display improvements in morphometric measurements, circulating metabolic markers, along with glucose tolerance and insulin sensitivity during weight loss from an obese (7 to 8) to moderate (5) body condition score (BCS). Effects of change in BCS or percent weight loss were evaluated using the mixed procedure of SAS with repeated measures. Change in BCS and percent weight loss were related to decreases in all morphometric measurements $(\mathrm{p}<0.05)$ except for abdominal circumference $(\mathrm{p}<0.10)$. Measurements of body composition also decreased with regard to both weight loss parameters $(\mathrm{p} \leq 0.001)$. Circulating markers of lipid metabolism revealed no changes in non-esterified fatty acid (NEFA) or triacylglyceride concentration, yet ex-vivo lipolysis media was decreased in response to weight loss $(\mathrm{p}=0.07)$ and BCS change $(\mathrm{p}=0.01)$. Insulin sensitivity calculated from baseline and post-weight loss intravenous glucose tolerance tests was improved after weight loss and corresponding BCS decline $(\mathrm{p} \leq 0.05)$ while the disposition index,
\end{abstract}


reflecting $\beta$-cell responsiveness, also increased due to weight loss $(\mathrm{p}=0.09)$. Lack of changes in circulating markers along with minimal changes in minimal model parameters suggest that while horses were obese, metabolic function was conserved. Further evaluation of metabolically healthy but obese horses may provide insight as to management and treatment of obesity associated maladies.

\section{Key Words}

Horse, weight loss, glucose, insulin, obesity

\section{Introduction}

Obesity among horses within the United States has reached an all time high, with nearly half of the nation's horses being considered either overweight or obese (Geor et al., 2007; Henneke et al., 1983). Obesity causes a multitude of metabolic issues in the horse, including impairment of glucose tolerance and insulin signaling, which compounded with compromised lipid metabolism and mitochondrial function can cause increased cellular damage and propagation of disease states. It has been well documented that obesity in the horse can cause impaired insulin signaling and glucose tolerance (Hoffman et al., 2003; Kronfeld, 2005), manifested by decreased insulin-stimulated glucose transport and metabolism in skeletal muscle and adipocytes (Kahn and Flier, 2000) and increased levels of circulating free fatty acids (Kahn and Flier, 2000). In combination with decreased GLUT4 transporter levels or translocation (Kahn and Flier, 2000) seen with an obese phenotype, dysfunction in the insulin signaling pathway is further propagated. As of now, the majority of studies completed evaluating these parameters have been 
conducted using two groups of animals: a lean and an obese group. While the effects of obesity on physiologic pathways have been studied, many areas still remain vague, particularly with regard to the lasting effects of obesity on metabolic function and stepwise alterations in metabolic capacity during weight loss. Often, weight loss, as a result of caloric restriction and/or exercise, is suggested to be the most beneficial method to decrease these effects of obesity; however, little is known about potential lasting effects of obesity on metabolic parameters after weight loss has occurred. Therefore; the objective of this study was to characterize the effects of weight loss on whole body markers of glucose and lipid metabolism and insulin sensitivity. Additionally, we sought to determine the effects of weight loss on cellular markers and regulators of glucose and lipid metabolism, including both $\beta$-oxidation and lipid biosynthesis. We hypothesized that weight loss would improve markers of insulin sensitivity and glucose tolerance, while decreasing levels of circulating triglycerides and increasing levels of non-esterified fatty acids. Further, we suspected lipolysis within the adipose tissue and $\beta$-oxidation of the lipids in skeletal muscle would increase.

\section{Materials and Methods}

1 The Middle Tennessee State University Institutional Animal Care and Use Committee

2 approved all methods and procedures used in this experiment. Horses were body

3 condition scored by two trained individual reviewers and horses assigned a body

4 condition score (BCS) of 7 or greater were admitted to the study. In addition, horses

5 selected for this screening process were known to not have any predisposing metabolic

6 conditions including Cushing's Disease or Metabolic Syndrome as defined for horses. 
7 Eight, healthy, mature (5-19 yrs) light type mixed sex ( $\mathrm{n}=6$ mares, $\mathrm{n}=2$ geldings) horses

8 of either Quarter Horse, Standardbred or Tennessee Walking Horse breeds from the

9 Middle Tennessee State University Horse Center herd were admitted based on these

10 parameters for use in this study (table 1).

\section{Sampling Periods and Diet}

13 Prior to and during the study, all horses received the same type of mixed grass hay and

14 commercial concentrate (Purina Strategy, Land O’Lakes Purina Mills, St. Louis, MO),

15 with ad libitum access to water. Dietary analysis was measured on concentrate samples

16 from 10 different bags and hay grab samples from at least 25 bales over 5 random dates

17 during the study (Equi-analyticial, Ithaca, NY, table 2 and 3). Further, study horses were

18 group housed in pastures with run-in shelters when not individually stalled over the entire

19 study. Pastures did contain some plant life; however, there was not enough coverage to

20 make a significant contribution to the diet and therefore was not included in digestable

21 energy (DE) intake calculations or dietary analysis. Due to limiting labor factors,

22 individual feed offerings and refusals were not measured daily, but estimated based on

23 average flake weight and concentrate cup weight, taken from 10 total measurements.

25 The baseline sampling period (BSP) began on $\mathrm{d} 0$ and continued until $\mathrm{d} 14$, during which

26 time horses were maintained on their current dietary intake, with a mean DE intake of

$2721.1 \mathrm{Mcal} / \mathrm{d}$. Upon entry into the weight loss period (WLP, d15), horses received a 30\%

28 DE reduction by altering amount of concentrate and hay offered. Horses remained on this

29 level of restriction until $\mathrm{d} 43$, at which time calculations were made for maintenance DE 
30 requirements based on current body weight, as described by the equation (NRC, 2007)

$31 \mathrm{DE}(\mathrm{Mcal} / \mathrm{d})=1.4+(0.03 \times \mathrm{BW})$ to achieve steady weight loss . In order to facilitate

32 further weight loss, a 30\% reduction from the maintenance requirement was calculated

33 and implemented throughout the remainder of the study (mean DE intake 14.01). At no

34 point during the study did horses consume less than $1.5 \% \mathrm{BW}$ in forage per day to ensure

35 proper hindgut health.

37 During both the BSP and the WLP, all horses were subjected to exercise not exceeding

38 the parameters of light workload as defined by the NRC. Horses were exercised on

39 average between 1-3 hr/wk, with an approximate gait breakdown of $40 \%$ walk, $50 \%$ trot

40 and $10 \%$ canter. Horses not broke to ride were lunged or worked in a round pen.

\section{Sample Acquisition \& Analysis}

43 Body Condition Score, Cresty Neck Score and Morphometric Measurements

44 Two individuals assigned a body condition score (BCS) based on a 1-9 scale (Henneke et

45 al., 1983) and a cresty neck score (CNS) on a 0-5 scale (Carter et al., 2009). Scores were

46 assigned on a whole or half score basis and the average was used for statistical analysis.

47 Morphometric measurements consisted of body weight, wither height, body length, neck

48 length (NL), neck crest height, neck circumference at $0.25,0.5$, and 0.75 of NL, girth

49 circumference, and abdominal circumference, in accordance with the measurements

50 made by Carter and colleagues (Carter et al., 2009). Measurements, BCS and CNS were

51 made on d $0,14,28,42,70$, and 98 . Body length was measured from the intermediate

52 tubercale of the humerous (point of the shoulder) to the ischiatic tuberosity (point of the 
53 buttock). All neck measurements were made with the neck in a relaxed position, at an

54 approximate $45^{\circ}$ angle. Neck length was measured from the poll to the highest point of

55 the wither. Neck crest height was measured at 0.5 neck length, from the differentiation of

56 musculature and tissue above the nuchal ligament (assessed by palpation and visual

57 identification) to the dorsal midline of the neck. Neck circumferences were measured at

$58 \quad 0.25,0.5$ and 0.75 of neck length. Girth circumference was measured immediately

59 behind the slope of the wither, caudal to the elbow (olecranon tuber). Abdominal

60 circumference was taken at two-thirds the distance from the intermediate tubercale of the

61 humerous (point of the shoulder) to the point of the hip (tuber coxae). Horses were

62 clipped in the areas where measurements were made initially to ensure later

63 measurements were taken at the same location.

64

\section{Body Composition Measurements}

66 Rump fat thickness (RFT) was measured using B-mode ultrasound (Sonovet 2000,

$675 \mathrm{mgHz}$ ) (Kearns et al., 2006a; Kearns et al., 2006b) on d 0, 14, 28, 42, 70, and 98. The

68 site for measurement was determined by measuring half the distance from the point of the

69 hip (tuber coxae) to the point of the buttock (ischiatic tuberosity); and half of the distance

70 from this point to the spine. Scans were made on alternating sides so as not to be affected

71 from healing biopsy sites. Body fat percentage was estimated using the following

72 equation (Kane et al., 1987); Percent Fat $=2.47+5.47$ (rump fat in $\mathrm{cm}$ ). Fat mass was

73 calculated by multiplying percent fat and total body mass. Fat free mass was determined

74 by the difference between total body mass and fat mass. 
77 Non-fasting blood samples $(\sim 35 \mathrm{~mL})$ were collected via jugular venipuncture on d 0,14 ,

$7821,28,35,42,56,70$, and 98 , placed into serum and sodium heparin vacutainers

79 (Vacutainer, Franklin Lakes, NJ). Sodium heparin tubes were immediately placed on ice,

80 while serum samples were allowed to clot at room temperature for at least 20 min before

81 being centrifuged at $3000 \mathrm{x} \mathrm{g}$ for $15 \mathrm{~min}$. Plasma and serum aliquots were removed and

82 stored at $-20^{\circ} \mathrm{C}$ until analysis. Serum non-esterified fatty acid (NEFA) and plasma

83 triacylglyceride (TG) concentrations were measured in duplicate using a commercially

84 available kit (HR-NEFA and L-Type TG Wako Diagnostics, Richmond, VA) as

85 instructed by the kit insert. The coefficient of variation accepted between duplicates was

$865 \%$ and 10\% for NEFA and TG respectively (O'Connor et al., 2007; O'Connor et al.,

87 2004). Glucose (Autokit glucose, Wako Diagnostics, Richmond, VA) and insulin (Coat-

88 A-Count Insulin, Siemens, Los Angeles, CA) concentrations were also determined using

89 commercially available kits, where the accepted coefficient of variation between

90 replicates was $5 \%$ and $10 \%$, respectively.

91

92 Frequently Sampled Intravenous Glucose Tolerance Tests (FSIGT)

93 All horses were subjected to a FSIGT on d 12 or 13 (end of BSP, FSIGT 1) and again at

94 the end of the WLP (FSIGT 2). As all horses did not exit the study on the same day,

95 FSGIT 2 was administered within $3 \mathrm{~d}$ of final morphometric measurements, biopsies, and

96 blood sampling. Concentrate feeding was withheld for at least $12 \mathrm{~h}$ prior to the test but

97 horses had access to grass hay the morning of and during the FSIGT. The protocol for 
98 this test was determined from previously published methods, described in detail below

99 (Hoffman et al., 2003).

101 Horses were weighed the morning of the test using an electronic scale and catheterized

102 (Abbocath, 14g, 5.5 in, Abbott Laboratories, Abbott Park, IL) using aseptic technique and

103 lidocaine anesthesia between 0800 and 0930. Horses were grouped randomly to allow for

104 test staggering if multiple horses were to be tested on the same day. A glucose bolus,

105 given at 0.3g/kg BW (50\% dextrose solution, Phoenix Pharmaceutical, Inc, St. Joseph,

106 MO), was infused via catheter within a 2 min period. Timing for all other samples began

107 after the infusion finished, where the 1 min sample signifies the collection of a sample 1

108 min post glucose infusion, et cetera. Twenty min post-glucose infusion, an exogenous 30

$109 \mathrm{mIU} / \mathrm{kg} \mathrm{BW}$ insulin bolus (Humulin R, Willow Birch Pharmaceutical, Inc, Taylor, MS)

110 was administered through the catheter. After each dose, the catheter, extension set and

111 stopcock were thoroughly flushed with heparinized saline.

113 The test was conducted over $\sim 4 \mathrm{~h}$, during which blood samples $(\sim 25 \mathrm{~mL})$ were collected

114 at the following time points: -30 (baseline), $0,1,2,3,4,5,6,7,8,9,10,12,14,16,19$,

$11522,23,24,25,27,30,35,40,50,60,70,80,90,100,120,150$ and 180 min post glucose

116 administration. An additional 240 min time point was sampled during FSIGT 1 to allow

117 adequate time for blood glucose to return to baseline for a more appropriate

118 representation of the data when completing Minimal Model analysis. 
120 Samples were collected into sodium heparin or serum tubes (Vacutainer, Franklin Lakes,

121 NJ). Sodium heparin tubes were placed immediately on ice while serum tubes were

122 allowed to clot at room temperature for at least $20 \mathrm{~min}$ before being placed on ice. All

123 tubes were centrifuged at $3000 \mathrm{x}$ g for $15 \mathrm{~min}$. Aliquots were removed and stored at $-20^{\circ}$

$124 \mathrm{C}$ until analysis. To determine insulin concentration, serum aliquots were analyzed in

125 duplicate using a commercially available radioimmunoassay (Coat-A-Count Insulin,

126 Siemens, Los Angeles, CA) as directed by the package insert. After the decanting step,

127 tubes were placed in a gamma counter (Perkin Elmer, Wallac 1470 Wizard Auto Gamma-

128 counter, Waltham, MA), where each replicate was counted for 1 min. Plasma aliquots

129 were measured in duplicate using a commercially available kit (Autokit Glucose, Wako

130 Diagnostics, Richmond, VA) adapted to a 96 well plate (Nielsen et al., 2010). In brief, 9

$131 \mu \mathrm{L}$ samples, standards, and controls were each loaded into the plate, followed by $300 \mu \mathrm{L}$

132 of buffer solution. Plates were then incubated for $2 \mathrm{~min}$, and read on a UV

133 spectrophotometer (Spectramax Plus 384, Molecular Devices, Sunnyvale, CA). Each of

134 these kits have been previously validated for equine samples (Hoffman et al., 2003). The

135 accepted coefficient of variation between replicates was 5\% and 10\% for glucose and

136 insulin, respectively (Nielsen et al., 2010). Area under the curve (AUC) for glucose and

137 insulin was calculated using the trapezoidal method, with individual data points from

138 each FSIGT (Nielsen et al., 2010). The Minimal Model Software Program (MinMod

139 Millenium, Version 6.2) (Boston et al., 2003) was used to calculate glucose effectiveness

140 (Sg), insulin sensitivity ( $\mathrm{Si}$ ), acute insulin response to glucose (AIRg) and disposition

141 index (DI). Baseline glucose (GB) and insulin (IB) are calculated using initial baseline

142 glucose and insulin values in addition to values of each curve after returning to baseline 
143 at the end of the test. The highest concentration of glucose after intravenous injection is

144 noted as G0. Any animal that did not fit the model with an $\mathrm{R}^{2}>0.9$ was removed from the

145 model prior to statistical analysis (FSIGT 1; n=1, FSIGT 2; n=0).

147 Neck Fat Biopsies

148 Neck fat biopsies were collected on d $0,14,28,42,70$, and 98. Prior to biopsy collection,

149 horses had biopsy sites clipped and scrubbed with chlorhexadine solution followed by an

150 isopropyl alcohol rinse. Horses were then sedated with xylazine $(0.5 \mathrm{mg} / \mathrm{kg} \mathrm{BW})$ and were

151 administered lidocaine anesthesia at the neck (2.5cc) site. Alternating sides were used for

152 consecutive biopsies so as to allow time for sites to fully heal before further sampling

153 occurred.

154

155 Neck fat biopsies were obtained at approximately 0.5 neck length (measured as described

156 above), centered between the differentiation of musculature and tissue above the nuchal

157 ligament using aseptic techniques (Liburt et al., 2012). A small incision, approximately 2

$158 \mathrm{~cm}$, was made through the skin and connective tissue to allow access to the neck fat pad.

159 An adequate sample was held with a hemostat and dissected out using a scalpel blade.

160 Incisions were closed with staples, left to heal for $10 \mathrm{~d}$, when staples were removed.

161 Samples were taken on alternating sides per biopsy to allow sites to fully heal. Post-

162 collection, adipose tissue samples were weighed and allocated for an ex vivo lipolysis

163 assay conducted immediately.

164

165 Lipolysis Assay 
166 The lipolysis assay was conducted as described previously (Ippagunta et al., 2011) with

167 slight modifications. A minimum of two $100 \mathrm{mg}$ pieces of adipose tissue were incubated

168 in Ringers buffer, with added BSA (fatty acid free, Sigma-Aldrich, St. Louis, MO) for $3 \mathrm{~h}$

169 at $37^{\circ} \mathrm{C}$ in a shaking water bath. Media sampled were collected and stored at $-20^{\circ} \mathrm{C}$ until

170 analysis for non-esterified fatty acids (NEFA, NEFA-HR kit, Wako, Richmond, VA) and

171 glycerol (free glycerol reagent, Sigma-Aldrich, Inc., St. Louis, MO) via colorimetric

172 analysis following the kit instructions. Accepted coefficient of variation between

173 duplicates was 5\% and 10\% for NEFA and glycerol, respectively. Concentrations of

174 NEFA and glycerol were adjusted for tissue weight and are expressed as mean $\mu \mathrm{mol}$

175 released per $\mathrm{g}$ of tissue.

176

177 Statistical Analysis

178 Data are expressed as mean \pm SEM. Normality of all data were assessed prior to

179 statistical analysis (SAS Institue, V9.3, Cary, NC). Any variable found to be non-

180 normally distributed by evaluation of kurtosis and skewness values, along with

181 significance in tests for location (students $t$ and sign tests) and normality (Shapiro-Wilk

182 and Klomogorov-Smirinov tests) where $\mathrm{p} \leq 0.05$, were $\log _{10}$ transformed. The effect of

183 BCS change and percent weight loss was tested for all data using proc MIXED procedure

184 of SAS with repeated measures. Gender, horse, age and date were included in the class

185 statement during the repeated measures analysis. The model with the best fit according to

186 Akaike's Information Criterion used a compound symmetry structure. A Pearsons

187 Correlation was run using the proc CORR procedure of SAS, with Bonferroni correction

188 to prevent the incidence of type I errors when performing multiple comparisons. For all 
189 data except correlations, $\mathrm{p}<0.05$ was considered significant and trends were noted at

$190 \mathrm{p}<0.10$. Due to the bonferroni correction, $\mathrm{p}<0.0032$ was considered significant and trends

191 noted when $\mathrm{p}<0.0047$ for all correlations.

192

\section{Results}

195 Body, Morphometric and Composition Measurements

196 A summary of individual horse characteristics including age, sex, breed, wither height,

197 body length and neck length can be found in table 4 . Horses on the study transitioned

198 from a mean BCS of $7.81 \pm 0.13$ to a moderate body condition, $5.0 \pm 0.0$ during the

199 course of the weight loss period. Change in body condition score and weight loss were

200 related to decreases in girth circumference $(\mathrm{p}<0.04)$, abdominal circumference $(\mathrm{p}<0.08)$,

201 cresty neck score $(\mathrm{p}<0.0001)$, neck crest height $(\mathrm{p}<0.0001)$, and all neck circumference

202 measurements $(\mathrm{p}<0.01$, table 5). Mean body weight decreased during the weight loss

203 period from $548.41 \pm 28.74 \mathrm{~kg}$ to $501.36 \pm 27.81 \mathrm{~kg}$, corresponding to decreasing BCS

204 during this time. Similarly, rump fat thickness, percent body fat, fat mass and fat free

205 mass decreased in relationship to both weight loss parameters $(\mathrm{p} \leq 0.001)$ and are

206 summarized by day in table 6 .

208 Circulating Metabolic Markers

209 Evaluation of serum NEFA (figure 1A) and plasma TG (figure 1B) as markers of

210 alterations in lipid metabolism revealed no differences due to change in BCS or percent

211 weight loss. As markers of carbohydrate metabolism, glucose concentration (figure 1C) 
212 displayed a significant increase when evaluated in relationship to change in BCS

213 ( $\mathrm{p}=0.01)$ but not with regard to percent weight loss. Conversely, insulin concentration

214 (figure 1D) did not differ with change in BCS, but displayed a trend when evaluated with

215 percent weight loss $(\mathrm{p}=0.06)$.

216

217 Lipolysis

218 Media collected from the lipolysis assay displayed a trending decrease in NEFA release

219 due to weight loss $(\mathrm{p}=0.07)$ and BCS change $(\mathrm{p}=0.01$, figure $2 \mathrm{~A})$. Free glycerol

220 concentration from the same samples did not differ due to either weight loss factor

221 ( $>>0.14$, figure 2B).

222

223 FSIGT and Minimal Model Parameters

224 Minimal model parameters calculated from FSIGT glucose and insulin concentrations

225 displayed variable results with regard to statistical model components (table 7). Glucose

226 effectiveness (Sg) or AIRg were not different due to weight loss parameters. Despite this

227 result, Si displayed an increase due to BCS change and percent weight loss $(\mathrm{p}<0.04)$. In

228 contrast, DI displayed no relationship to change in BCS, yet a trend was noted with

229 regard to percent weight loss during the study $(\mathrm{p}=0.09)$. Baseline glucose $(\mathrm{GB})$, as

230 calculated from beginning and end of the FSIGT decreased, with a trend noted for BCS

231 change $(\mathrm{p}=0.06)$ and a significant difference in response to weight loss percentage

$232(\mathrm{p}=0.002)$. Baseline insulin (IB) and $\mathrm{G} 0$, the highest concentration of glucose post-

233 intravenous injection, were not different due to either model parameter for weight loss

$234(\mathrm{p}>0.21)$. 
236 Area under the curve for glucose and insulin during the FSIGT was calculated utilizing

237 the trapezoidal method, and analyzed in accordance with other variables. Glucose AUC

238 (figure 3A) decreased in response to percent weight loss $(\mathrm{p}=0.0004)$ and change in BCS

$239(\mathrm{p}=0.05)$. A similar effect was seen for insulin AUC (figure 3B), where when analyzed

240 with regard to BCS change, a significant difference was detected $(\mathrm{p}=0.02)$; however,

241 when analyzed for effect of percent weight loss, no difference was found.

\section{Correlations}

244 Correlations between collected data were analyzed in two separate sets: (1) analyzing all

245 morphometric measurements, body composition data, circulating metabolic markers and

246 lipolysis media concentrations of NEFA and FG, and (2) analyzing only data collected on

247 days 14 and 96 including all minimal model parameters in accordance with the dates

248 FSIGT were completed. A summary of correlations described in set 1 can be found in

249 table 7. Weight was found to be correlated to girth circumference ( $\mathrm{r}=0.84)$, abdominal

250 circumference $(\mathrm{r}=0.62)$, neck circumference at $0.25(\mathrm{r}=0.79), 0.5(\mathrm{r}=0.69)$, and $0.75 \mathrm{NL}$

251 ( $\mathrm{r}=0.76)$, and fat free mass $(\mathrm{r}=0.99)$, where $\mathrm{p}<0.0001$ for all correlations. A trend was

252 also noted for the correlation between fat mass and weight $(r=0.42, p=0.003)$. As body

253 condition score decreases, a trending correlation with decreasing lipolysis NEFA

254 concentration $(\mathrm{r}=0.43, \mathrm{p}=0.004)$ was displayed.

255

256 Many of the same correlations were noted when comparing the aforementioned variables

257 with minimal model parameters; however; due to a more stringent Bonferronis correction 
258 as more variables were included in the correlation, only a few significant relationships

259 were determined. Body condition score $(r=0.75, p=0.001)$ and cresty neck score $(r=0.73$,

$260 \mathrm{p}=0.001$ ) were found to have positive correlations with AUC glucose. Area under the

261 insulin FSIGT curve displayed positive correlations with fat mass $(r=0.71, p=0.0019)$ and

$262 \mathrm{~Gb}(\mathrm{r}=0.80, \mathrm{p}=0.0002$, data not shown).

\section{Discussion}

265 Body composition is an important factor to consider when evaluating metabolic function,

266 specifically when related to glucose metabolism (Pedersen et al., 2003; Reaven, 1995).

267 Horses included in the current study displayed decreases in all morphometric and body

268 composition measurements during weight loss. As the Henneke body condition scoring

269 system is subjective and can vary slightly due to each independent assessor (Henneke et

270 al., 1983), morphometric measurements were also included to provide a more

271 quantitative, comprehensive representation of regional adiposity differences during

272 weight loss (Carter et al., 2009). Baseline mean cresty neck scores were similar to those

273 found in insulin resistant ponies (Tinworth et al., 2012); yet overall BCS of the ponies

274 was $6.0 \pm 0.9$, signifying a greater amount of regional adiposity than our horses which

275 were at a greater BCS. In accordance with decreasing body condition score, all

276 morphometric measurements including girth circumference, neck crest height, and 0.25,

$277 \quad 0.5$ and 0.75 neck circumference decreased.

278

279 Rump fat thickness is a measured parameter directly reflecting fat mass in the body

280 (Westervelt et al., 1976). The concurrent decrease in rump fat with body weight suggests 
281 horses were loosing fat mass during the course of the study. Calculations of fat mass and

282 fat free mass also displayed patterns reflecting this concept, whereas body weight

283 decreased, fat mass decreased from $46.37 \pm 4.39$ to $29.11 \pm 3.10 \mathrm{~kg}$ and fat free mass

284 declined $502.04 \pm 27.14$ to $472.25 \pm 26.54 \mathrm{~kg}$. The decrease in fat free mass suggests

285 utilization of lean body mass sources for energy homeostasis consistent with calorie

286 restriction. The light exercise program was not rigorous enough to increase muscle mass

287 and as horses were accustomed to this level of activity no muscular adaptation to

288 workload was expected.

289

290 Compared to other morphometric measurements, abdominal circumference displayed the

291 smallest decrease over time, which may play a role in the lack of fluctuations seen in

292 metabolic markers and minimal model parameters. In humans, intraabdominal fat

293 deposits appear to have a direct role in disrupting glucose and lipid homeostasis (Leibel

294 et al., 1989). As intraabdominal deposits drain directly into the portal vein, thereby

295 resulting in high concentrations of hepatic free fatty acids which may interfere with

296 hepatic catabolism of insulin along with overproduction of very low density lipoproteins

297 (Leibel et al., 1989; Smith et al., 1985). The resultant increase in circulating insulin

298 concentration may propagate insulin insensitivity over time. It is possible that during the

299 process of fat deposition in the horse, abdominal fat is deposited last and also

300 metabolized later during the weight loss process. In a study evaluating fat deposition in

301 response to different dietary sources, the greatest amount of fat lay down occurred in the

302 neck, with the smallest amount over the withers and loin (Suagee et al., 2008).

303 Comparing this concept to neck crest fat, where a higher CNS is often linked to metabolic 
304 syndrome predisposition (Carter et al., 2009; Treiber et al., 2006), it is plausible that due

305 to the moderate CNS scores (2-3) seen during this study, the horses had not deposited

306 enough fat to cause alterations in many of the metabolic parameters measured.

308 Additionally, specific fat deposits have varying metabolic functions and therefore may

309 contribute differently to energy homeostasis during weight loss (Liburt et al., 2012).

310 Within the horse, it is possible that the role of neck fat relating to homeostatic function

311 gives an accurate yet localized overview of adipose tissue function in this model. As the

312 neck fat crest is easy to access for sampling purposes, many groups have analyzed the

313 metabolic function of this fat deposit (Liburt et al., 2012). Despite its value due to ease of

314 accessibility, analysis of other fat deposits may be instrumental to fully elucidating the

315 contributions of each deposit and changes associated with weight loss within adipose

316 tissue. We found a decrease in NEFA release due to both weight loss parameters but no

317 change in free glycerol during our measurement of lipolysis. These differences may be

318 due to the 3 to 1 ratio of fatty acids to the glycerol backbone in TG (Ippagunta et al.,

319 2011). Combined with the lack of differences in serum NEFA and plasma TG with either

320 weight loss parameter, it is possible that while horses were overweight, the excess

321 condition did not cause unhealthy lipid metabolism.

323 Additionally, a potential explanation for the lack of differences in circulating NEFA and

324 TG values is that horses were not fasted at the time of sampling. Samples obtained from

325 non-fasting horses displayed lower levels of NEFA (O'Connor et al., 2007) due to

326 alterations between fed and fasting state metabolism. While most of the horses on the 
327 study were receiving mainly grass hay and a very small quantity of concentrate, the

328 possibility remains that if horses had been sampled prior to morning feed times different

329 results may have been displayed. This would explain the elevated glucose concentrations

330 that would otherwise suggest horses were hyperglycemic both during baseline and post-

331 weight loss. Despite the significant difference in glucose concentration due to change in

332 BCS and the trend noted for insulin concentration in relationship to percent weight loss, it

333 is difficult to determine if this was a true weight loss response or a result of time of

334 sampling post feeding. A more accurate representation of glucose and insulin

335 concentrations due to weight loss is observed during the FSIGT, and will be discussed

336 further.

338 As a better representation of fasting glucose concentration, $\mathrm{Gb}$ calculated from beginning

339 and end of each FSIGT glucose concentrations, displayed significant decreases due to

340 both weight loss and BCS change. This suggests that while initial alterations to glucose

341 metabolism may have been occurring, they had not advanced to implicating other

342 pathways measured. Upon evaluation of minimal model parameters along with glucose

343 and insulin curve responses it is apparent that large individual variability is observed.

344 While Si displayed an improvement, the lack of change in Sg, or AIRg suggest that while

345 horses were obese, carbohydrate metabolic function was not entirely compromised.

346 Increases in $\mathrm{Si}$, the capacity for insulin to promote glucose disposal (Hoffman et al.,

347 2003), can be contributed to weight loss, as in previous studies low intensity exercise

348 alone did not produce improvements in Si (Carter et al., 2010). Interestingly, AIRg values

349 observed during baseline when horses were obese are similar to those of moderately 
350 obese horses (BCS 6-6.9) observed by Hoffman and colleagues (Hoffman et al., 2003).

351 After weight loss, AIRg values were consistent with those reported in literature for horses

352 with a BCS range of 5-5.9 (Hoffman et al., 2003). The trend observed between DI and

353 percent weight loss may represent initial changes in endogenous insulin release, which

354 may have been amplified if a larger sample size had been used. Previously reported

355 literature demonstrates DI between horses of non-obese (BCS 5.0-5.9) and obese (BCS 7-

356 9) condition did not differ, suggesting adequate ability in obese animals for AIRg to

357 compensate for limited Si (Hoffman et al., 2003); a phenomenon which also may be

358 present as horses transition through decreasing body condition scores.

360 Some variability between FSIGT 1 and 2, along with intraassay variation may

361 additionally contribute to the lack of difference between FSIGT dates. In a previous study

362 comparing multiple methods for determining insulin sensitivity and glucose tolerance,

363 repeated tests displayed $\mathrm{Si}, \mathrm{Sg}$ and $\mathrm{AIRg}$ mean coefficient of variation of $24 \%, 26 \%$ and

$36412 \%$, respectively (Pratt et al., 2005). While utilization of the FSIGT and minimal model

365 analysis is still an accurate measurement of glucose and insulin dynamics, it is important

366 to consider the role of variability when interpreting results. In order to represent

367 individual horse differences in FSIGT responses during baseline (date 1) and after weight

368 loss (date 2) figure 4 depicts glucose (A) and insulin (B) curves. Horse numbers

369 correspond to those in table 1 . Horse variability greatly influenced the results of this

370 study. Although no statistical differences were noted for many of the minimal model

371 parameters, the physiological implications of individual horse responses to the FSIGT

372 hold value in understanding how the body responds to decreases in body fat and weight. 
373 When evaluated as a whole, significant differences in area under the curve for both

374 glucose and insulin concentrations suggest that horses were compromised in some aspect

375 of carbohydrate metabolism, but not to the extent to produce statistical differences in

376 minimal model compartment parameters.

377

378 When evaluating the overall implications of this study, it remains plausible that while

379 horses were considered obese at the start of the study, from a metabolic perspective they

380 were not unhealthy. The term "metabolically healthy obese" has been coined in human

381 health to describe obese individuals exhibiting relatively normal metabolic functions

382 despite being as overweight as those presenting with metabolic dysfunction and

383 nutritional diseases (Bonora et al., 1991; Sims, 2001; Wildman et al., 2008). It is

384 hypothesized that the incidence of this phenotype is relatively prevalent, with up to $30 \%$

385 of the adult obese population possessing reduced central adiposity, lower adipocyte stress

386 and decreased inflammation (Denis and Obin, 2013; Kloting et al., 2010). While insulin

387 action is preserved in these subjects despite increasing body mass index (Bluher, 2012;

388 Bluher, 2010; Kloting et al., 2010), it is not yet understood whether these individuals are

389 genetically predisposed to maintain insulin sensitivity or whether the phenotype is

390 inherently unstable and can evolve rapidly to an insulin resistant state (Kim and Reaven,

391 2008). Despite horses included in this study beginning at an obese BCS, relatively

392 moderate CNS scores may signify a similar phenomenon within the selected population.

393 Horses with a greater adipose deposition may produce a different outcome, as horses may

394 transition into an unhealthy phenotype during this time. By selecting for horses without

395 history of laminitic issues or known insulin resistance, we may have chosen a subset of 
396 horses with resistance to developing metabolic dysfunctions. Moreover, this may suggest

397 that while obesity is not a desired phenotype for the horse, that animals enrolled in an

398 exercise program may be additionally protected from the onset of insulin resistance and

399 other obesity-related metabolic maladies.

400

401 Looking forward, increasing the sample size for a study evaluating weight loss markers

402 may be necessary to both eliminate confounding effects of horse-to-horse variability

403 along with obtaining samples both during weight loss and weight gain. As many of the

404 studies involving FSGIT with minimal model analysis and other metabolic studies have

405 used between 6-10 horses, we suspected that this sample number would be adequate to

406 elucidate changes due to weight loss as well (Hoffman et al., 2003; Liburt et al., 2012;

407 Pratt et al., 2005; Tinworth et al., 2012). Also, collected data from this study could be

408 utilized for a power analysis to determine sample number for future work in this area.

410 In conclusion, horses may exhibit a metabolically preserved obesity phenotype that is

411 relatively protected from alterations in glucose and lipid metabolism. Deposition of fat

412 more equally throughout the body as opposed to large deposits in the neck crest or

413 intraabdominal regions may contribute to this phenomenon. Additionally modifications to

414 insulin sensitivity and thus glucose and insulin concentrations in response to weight loss

415 during an FSIGT may signify beginning metabolic changes that if horses were allowed to

416 deposit more adipose tissue may cause further impairment over time. Future studies are

417 needed to examine effects of the length of time in an obese state as well as stepwise 
418 changes in weight gain in order to fully elucidate mechanisms behind obesity induced

419 metabolic dysfunction.

420

421 Acknowledgements

422 Funding for this project provided in part by the West Virginia University Summer

423 Undergraduate Research Experience Program and Middle Tennessee State University.

424 Special recognition is warranted for the Middle Tennessee State University Horse

425 Science Center employees, including Jessica Shultz, Jeremy Carlson, Renee Dunn, Dr.

426 John Haffner, the student farm workers, and the horse program graduate students for their

427 assistance in the completion of this project.

428

429 
Tables and Figures

431 Table 1: Summary of individual characteristics of horses enrolled in study

\begin{tabular}{ccccccc}
\hline \hline Horse & Age (yr) & Sex $^{1}$ & Breed & $\begin{array}{c}\text { Wither } \\
\text { Height } \\
(\mathrm{cm})^{2}\end{array}$ & $\begin{array}{c}\text { Body } \\
\text { Length } \\
(\mathrm{cm})^{3}\end{array}$ & $\begin{array}{c}\text { Neck } \\
\text { Length } \\
(\mathrm{cm})^{4}\end{array}$ \\
\hline 1 & 10 & $\mathrm{M}$ & Quarter Horse & 142 & 158 & 71 \\
2 & 6 & $\mathrm{M}$ & Tennessee Walker & 148 & 137 & 71 \\
3 & 13 & $\mathrm{M}$ & Standardbred & 160 & 156 & 101 \\
4 & 19 & $\mathrm{G}$ & Quarter Horse & 149 & 150 & 86 \\
5 & 5 & $\mathrm{M}$ & Quarter Horse & 147 & 152 & 81 \\
6 & 10 & $\mathrm{G}$ & Quarter Horse & 170 & 165 & 97 \\
7 & 19 & $\mathrm{M}$ & Quarter Horse & 151 & 159 & 95 \\
8 & 15 & $\mathrm{M}$ & Quarter Horse & 157 & 165 & 104 \\
\hline & & & Mean \pm SEM & $153 \pm 3$ & $155 \pm 3$ & $88 \pm 4$ \\
\cline { 3 - 6 } & & & & &
\end{tabular}

432

$433{ }^{1}$ For sex categorization $M$ represents mare and $\mathrm{G}$ represents gelding.

$434 \quad{ }^{2}$ Measured from the ground to the highest point of the wither

4353 Measured from the point of the shoulder to the point of the rump

4364 Measured from the poll to the point of union between neck musculature and the wither 437

438 
439 Table 2: Nutrient analysis of grass hay and concentrate, as fed

\begin{tabular}{lcc}
\hline \hline Nutrient, as fed & Grass Hay & Concentrate \\
\hline Dry Matter (\%) & 95.8 & 93.4 \\
Digestable Energy (DE, Mcal/kg) & 1.85 & 2.84 \\
Crude Protein (g/kg) & 51.5 & 150.1 \\
Acid Detergent Fiber (ADF, g/kg) & 371.8 & 166 \\
Neutral Detergent Fiber (NDF, g/kg) & 625.8 & 330.8 \\
Water Soluble Carbohydrates (WSC, g/kg) & 56.0 & 89.4 \\
Ethanol Soluble Carbohydrates (ESC, g/kg) & 15.5 & 51.7 \\
Starch (g/kg) & 14.3 & 131.4 \\
Non-fiber Carbohydrates (NFC, g/kg) & 156.8 & 350.9 \\
Crude Fat (g/kg) & 28.6 & 59.3 \\
Calcium (g/kg) & 6.03 & 12.91 \\
Phosphorus (g/kg) & 0.83 & 8.15 \\
Sodium (g/kg) & 0.218 & 2.369 \\
\hline
\end{tabular}

440

441 Table 3: Percent composition of diet on a dry matter basis

\begin{tabular}{lcr}
\hline \hline Nutrient, \% dry matter & Grass Hay & Concentrate \\
\hline Crude Protein, \% & 5.4 & 16.1 \\
Acid Detergent Fiber, \% & 38.8 & 17.8 \\
Neutral Detergent Fiber, \% & 65.4 & 35.4 \\
Water Soluble Carbohydrates, \% & 5.9 & 9.6 \\
Ethanol Soluble Carbohydrates, \% & 1.6 & 5.5 \\
Starch, \% & 1.5 & 14.1 \\
Non-fiber Carbohydrates, \% & 16.4 & 37.6 \\
Crude Fat, \% & 3 & 6.3 \\
Calcium, \% & 0.63 & 1.38 \\
Phosphorus, \% & 0.09 & 0.87 \\
Sodium, \% & 0.023 & 0.254 \\
\hline
\end{tabular}


444 Table 4: Morphometric measurements during weight loss

\begin{tabular}{|c|c|c|c|c|c|c|c|c|c|}
\hline \multirow{3}{*}{ Variable } & & & & & & & & \multicolumn{2}{|c|}{ P-Value } \\
\hline & & \multicolumn{6}{|c|}{ Day } & $\begin{array}{l}\text { Change } \\
\text { in } B C S\end{array}$ & $\begin{array}{l}\text { Percent } \\
\text { Weight }\end{array}$ \\
\hline & & 0 & 14 & 28 & 42 & 70 & 98 & & \multirow{3}{*}{. } \\
\hline \multirow{2}{*}{$\begin{array}{l}\text { Body Condition } \\
\text { Score }^{1}\end{array}$} & Mean & 7.81 & 7.75 & 7.44 & 7.38 & 6.13 & 5.00 & & \\
\hline & SEM & 0.13 & 0.13 & 0.18 & 0.23 & 0.25 & 0.00 & & \\
\hline \multirow{2}{*}{$\begin{array}{c}\text { Girth } \\
\text { Circumference }^{2,3}\end{array}$} & Mean & 194 & 195 & 194 & 193 & 193 & 189 & \multirow[t]{2}{*}{0.04} & \multirow[t]{2}{*}{0.01} \\
\hline & SEM & 4 & 4 & 4 & 4 & 5 & 3 & & \\
\hline \multirow{2}{*}{$\begin{array}{c}\text { Abdominal } \\
\text { Circumference }\end{array}$} & Mean & 215 & 212 & 208 & 206 & 202 & 199 & \multirow[t]{2}{*}{0.08} & \multirow[t]{2}{*}{0.06} \\
\hline & SEM & 4 & 4 & 4 & 10 & 7 & 5 & & \\
\hline \multirow{2}{*}{$\begin{array}{l}\text { Cresty Neck } \\
\text { Score }^{5}\end{array}$} & Mean & 2.69 & 2.63 & 2.64 & 2.56 & 2.19 & 2.07 & \multirow[t]{2}{*}{$<0.0001$} & \multirow[t]{2}{*}{$<0.0001$} \\
\hline & SEM & 0.09 & 0.08 & 0.13 & 0.15 & 0.09 & 0.07 & & \\
\hline \multirow{2}{*}{$\begin{array}{l}\text { Neck Crest } \\
\text { Height }^{2.6}\end{array}$} & Mean & 12 & 12 & 10 & 9 & 9 & 7 & \multirow[t]{2}{*}{$<0.0001$} & \multirow[t]{2}{*}{$<0.0001$} \\
\hline & SEM & 1 & 1 & 1 & 1 & 1 & 1 & & \\
\hline \multirow{2}{*}{$\begin{array}{l}\text { Neck Circ. at } \\
0.25 \mathrm{NL}^{2,7}\end{array}$} & Mean & 79 & 80 & 78 & 77 & 77 & 74 & \multirow[t]{2}{*}{0.002} & \multirow[t]{2}{*}{0.01} \\
\hline & SEM & 2 & 1 & 2 & 2 & 1 & 2 & & \\
\hline \multirow{2}{*}{$\begin{array}{l}\text { Neck Circ. at } \\
0.5 \mathrm{NL}^{, 7}\end{array}$} & Mean & 101 & 99 & 94 & 94 & 96 & 91 & \multirow[t]{2}{*}{0.003} & \multirow[t]{2}{*}{0.002} \\
\hline & SEM & 3 & 3 & 2 & 2 & 3 & 1 & & \\
\hline \multirow{2}{*}{$\begin{array}{c}\text { Neck Circ. at } \\
0.75 \mathrm{NL}^{2,7}\end{array}$} & Mean & 118 & 117 & 112 & 110 & 114 & 108 & \multirow[t]{2}{*}{0.003} & \multirow[t]{2}{*}{$<0.0001$} \\
\hline & SEM & 3 & 3 & 3 & 3 & 4 & 3 & & \\
\hline
\end{tabular}

445

$446{ }^{1}$ Assessment based on a 1-9 scale (Henneke et al., 1983)

$447 \quad{ }^{2}$ All measurements made in $\mathrm{cm}$

$448{ }^{3}$ Measured immediately behind slope of wither, caudal to elbow

$449{ }^{4}$ Measured at two thirds distance from point of shoulder to point of hip

$450 \quad{ }^{5}$ Assessment based on 1-5 scale (Carter et al., 2009) 
$451{ }^{6}$ Measured at 0.5 neck length (measured from poll to highest point of the wither), from

452 the differentiation of musculature and tissue above the nuchal ligament (discerned from

453 visual and palpation assessment)

$454{ }^{7}$ Neck circumferences taken at $0.25,0.5$ and 0.75 neck length (measured from poll to 455 highest point of the wither)

456 
457 Table 5: Association of body fat measurements with BCS change and percent weight loss 458

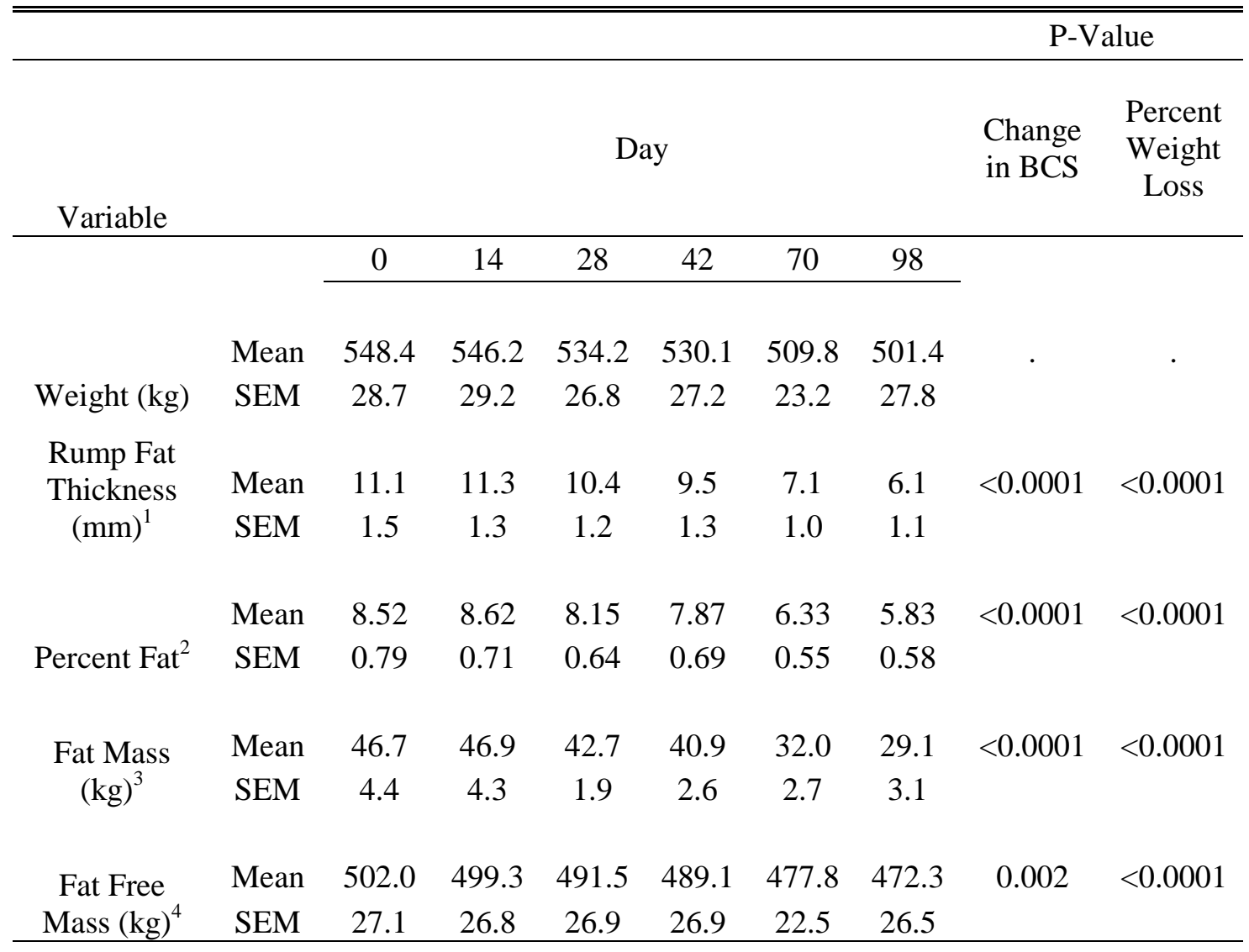

459

$460{ }^{1}$ Measured by B-mode ultrasound, site determined by measuring half the distance from

461 the point of the hip (tuber coxae) to the point of the buttock (ischiatic tuberosity); and

462 half of the distance from this point to the spine, units expressed in $\mathrm{cm}$

$463{ }^{2}$ Percent fat $=2.47+(5.47 \mathrm{x}$ rump fat thickness in $\mathrm{cm})$

$464 \quad{ }^{3}$ Fat mass $=$ percent fat $\mathrm{x}$ body weight

$465{ }^{4}$ Fat free mass = body weight - fat mass

466

467 
468 Figure 1 A-D: Effects of decreasing body condition and percent weight loss on circulating metabolic indicators. Horses $(n=8)$ were enrolled in a baseline-sampling period lasting from $\mathrm{d} 0$ to $\mathrm{d} 14$, followed by a caloric restriction from $\mathrm{d} 15$ to $\mathrm{d} 98$, where from $\mathrm{d} 15$ to $\mathrm{d} 43$ horses received a $30 \%$ digestible energy reduction, and from d44 to d 98 horses received a $30 \%$ restriction from maintenance digestible energy. Non-fasting blood samples were taken on d $0,14,21,28,35,42,56,70$, and 98 and were analyzed for serum non-esterified fatty acids (NEFA) and insulin, along with plasma triacylglycerides (TG) and glucose concentrations. The main effect of change in body condition score (BCS) and percent weight loss were analyzed for each variable, with data points
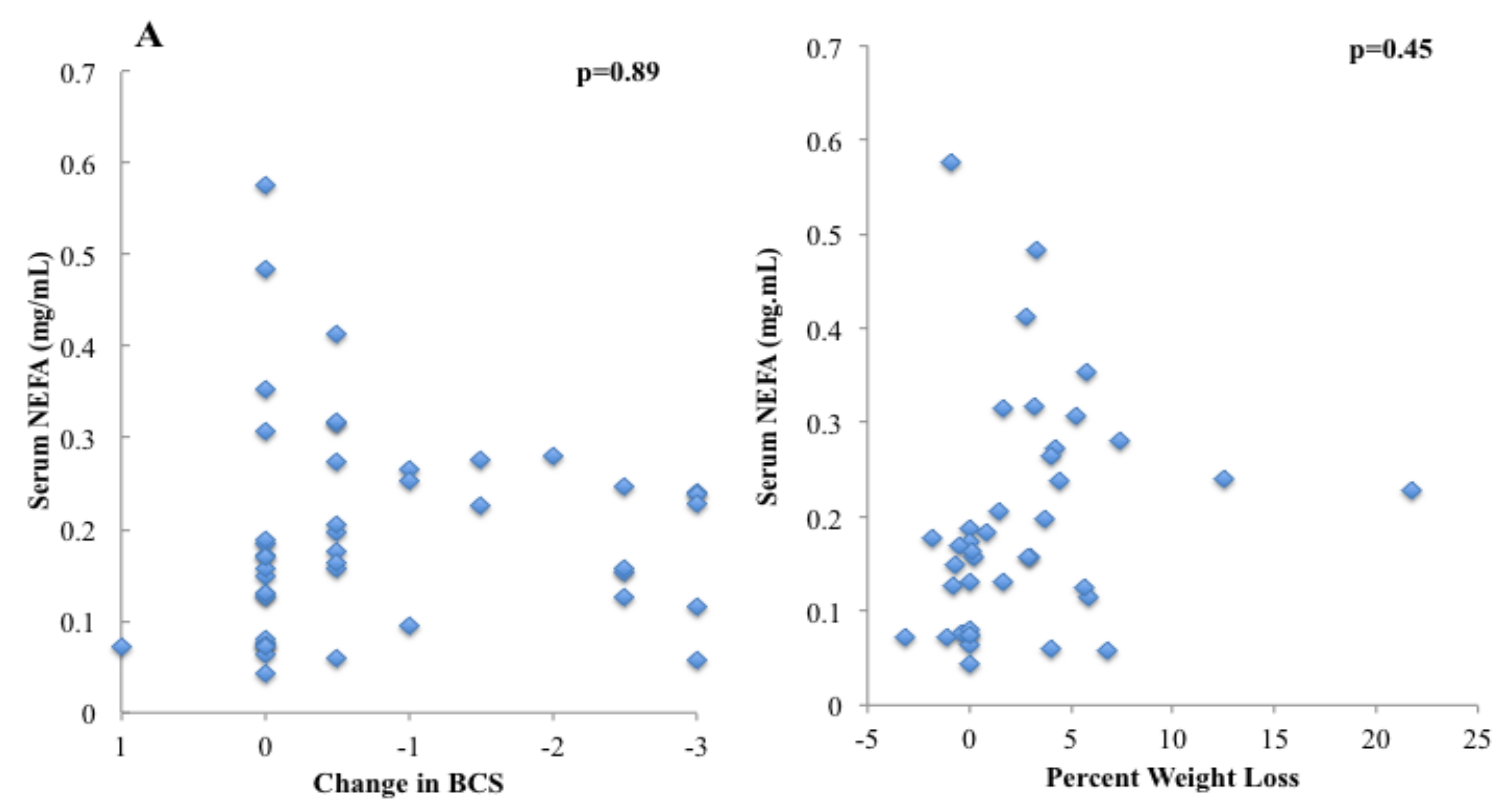

481
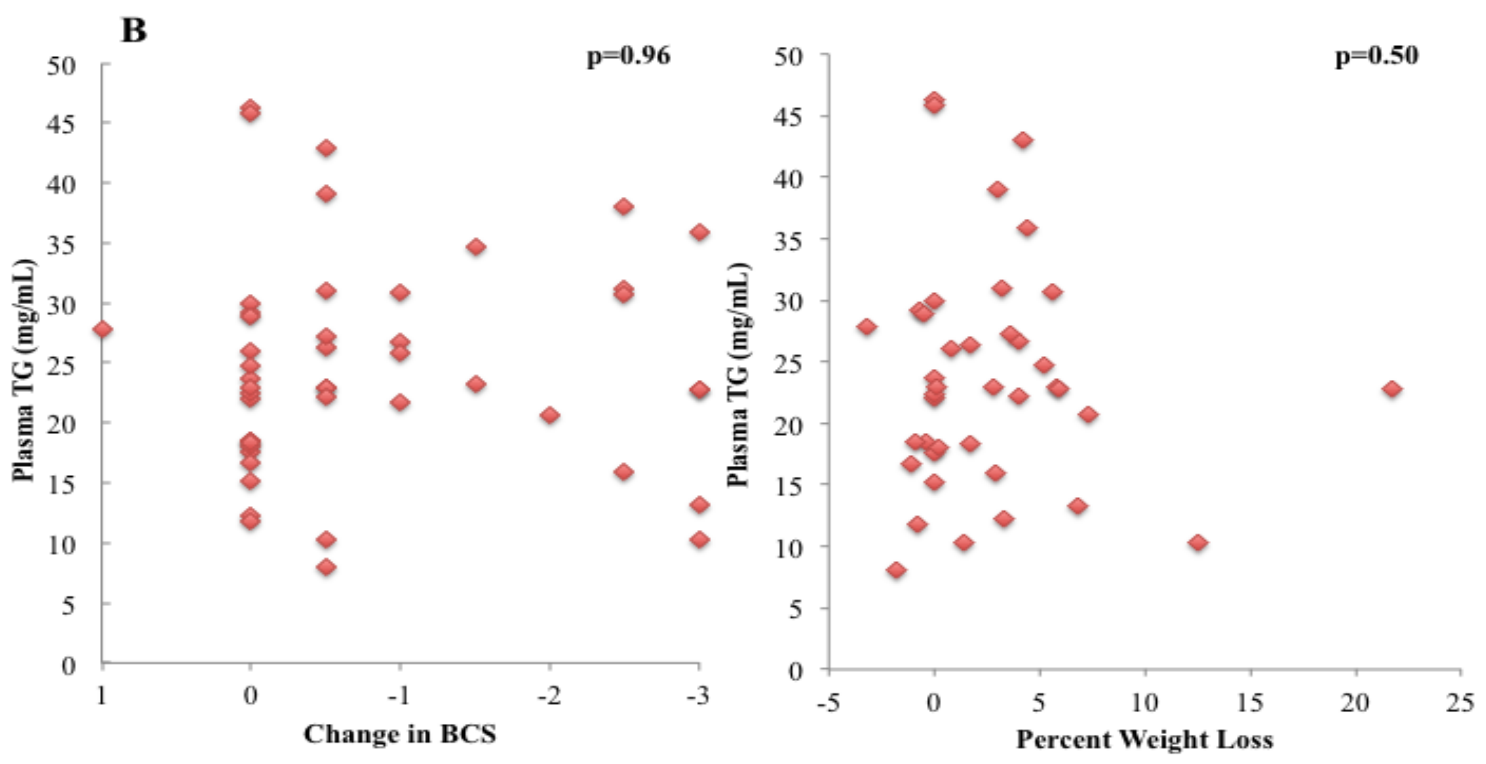

483 
484 Figure 1 A-D: Effects of decreasing body condition and percent weight loss on 485 circulating metabolic indicators. Horses $(n=8)$ were enrolled in a baseline-sampling 486 period lasting from $\mathrm{d} 0$ to $\mathrm{d} 14$, followed by a caloric restriction from $\mathrm{d} 15$ to $\mathrm{d} 98$, where 487 from $\mathrm{d} 15$ to $\mathrm{d} 43$ horses received a $30 \%$ digestible energy reduction, and from d44 to d 98 488 horses received a $30 \%$ restriction from maintenance digestible energy. Non-fasting blood samples were taken on d $0,14,21,28,35,42,56,70$, and 98 and were analyzed for serum non-esterified fatty acids (NEFA) and insulin, along with plasma triacylglycerides (TG) and glucose concentrations. The main effect of change in body condition score 493 (BCS) and percent weight loss were analyzed for each variable, with data points
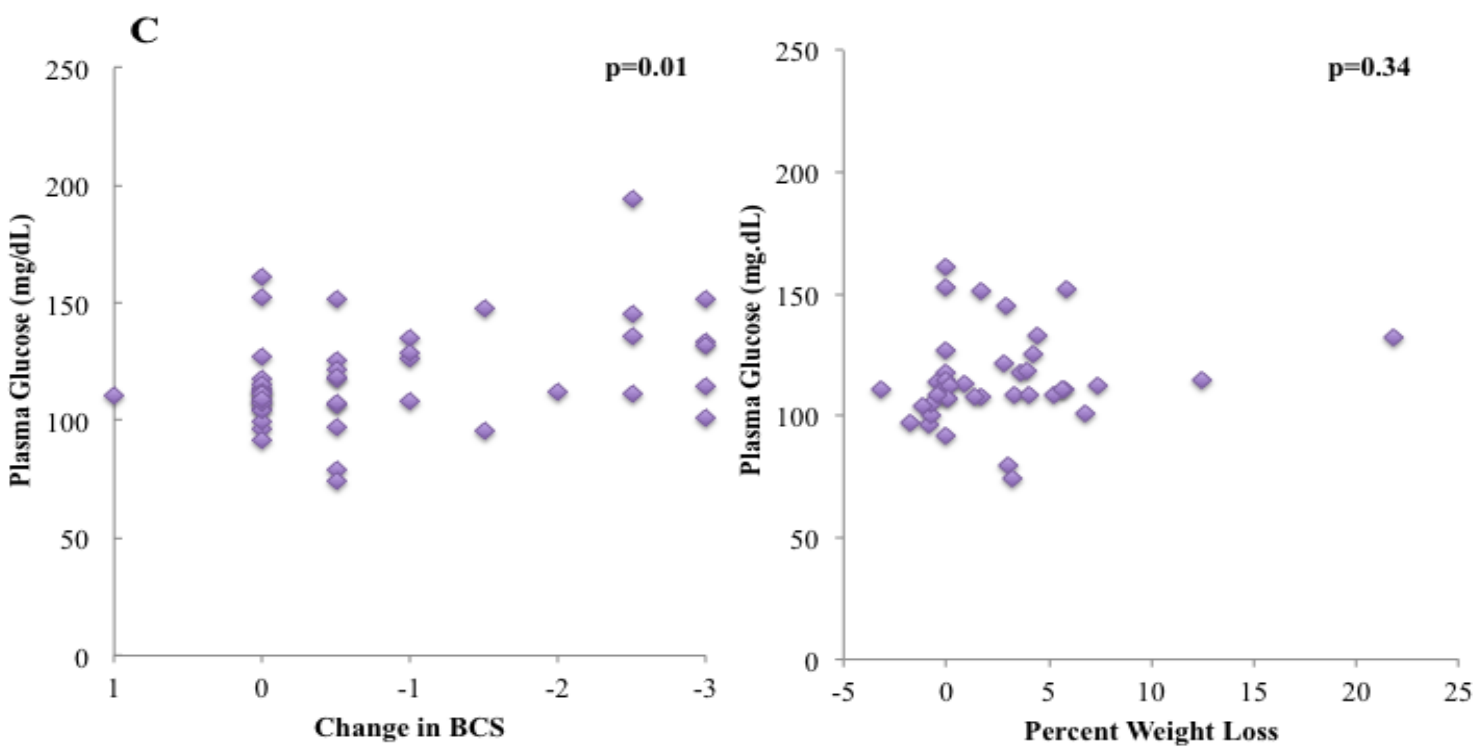

495
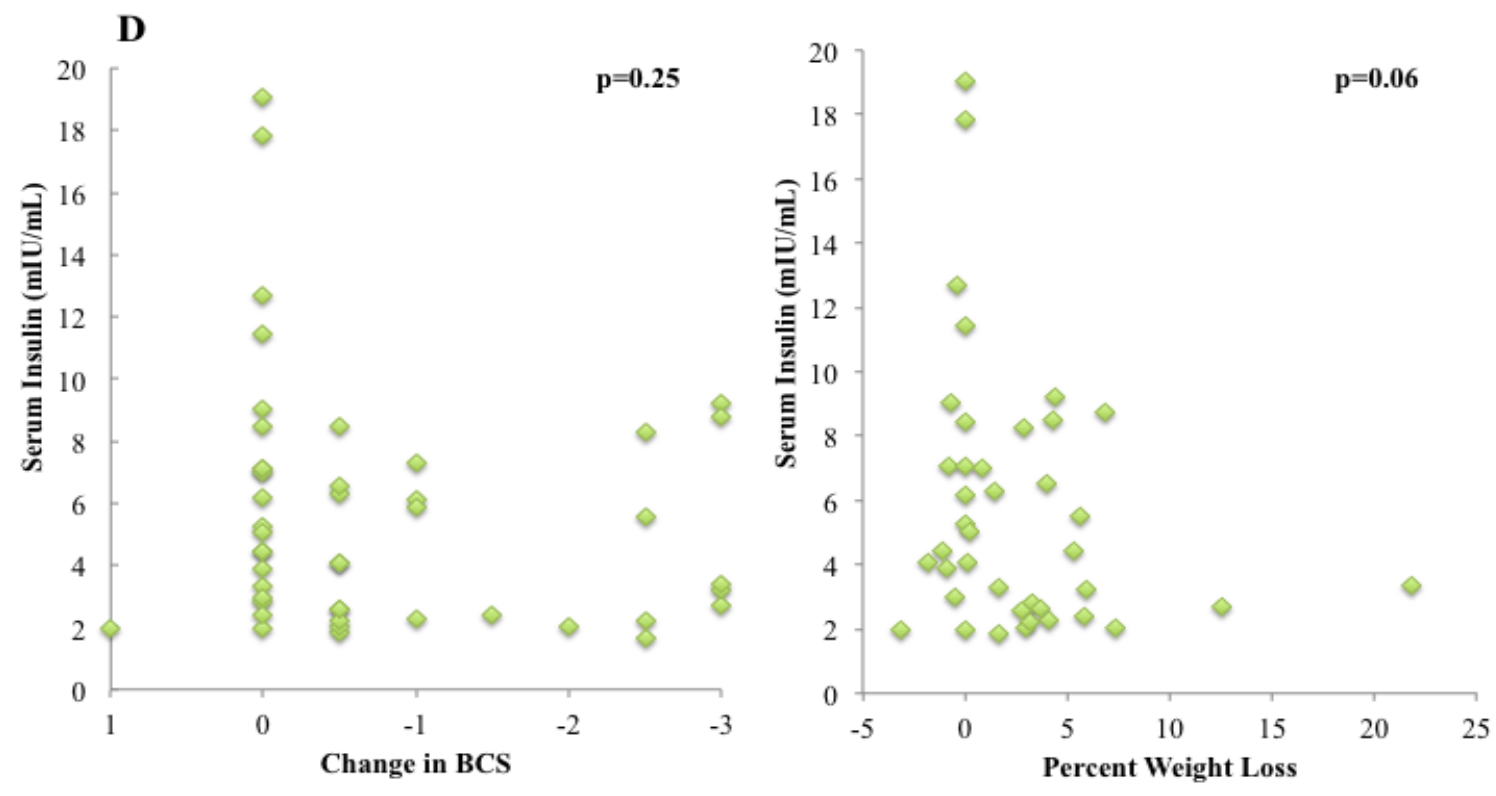

497

498 
499 Figure 2 A-B: Influence of weight loss on lipolysis markers. Horses $(\mathrm{n}=8)$ were 500 enrolled in a baseline-sampling period lasting from $\mathrm{d} 0$ to $\mathrm{d} 14$, followed by a caloric 501 restriction from $\mathrm{d} 15$ to $\mathrm{d} 98$, where from $\mathrm{d} 15$ to $\mathrm{d} 43$ horses received a $30 \%$ digestible 502 energy reduction, and from $\mathrm{d} 44$ to $\mathrm{d} 98$ horses received a $30 \%$ restriction from 503 maintenance digestible energy. On d 0, 14, 28, 42, 70, and 98, a neck fat biopsy was 504 collected and immediately allocated for an ex vivo lipolysis assay. Media collected from 505 the assay was analyzed for non-esterified fatty acids (NEFA) and free glycerol 506 concentrations. The main effect of change in body condition score (BCS) and percent 507 weight loss were analyzed for each variable, with data points representing values at 508 corresponding points of BCS change or weight loss, respectively.

509
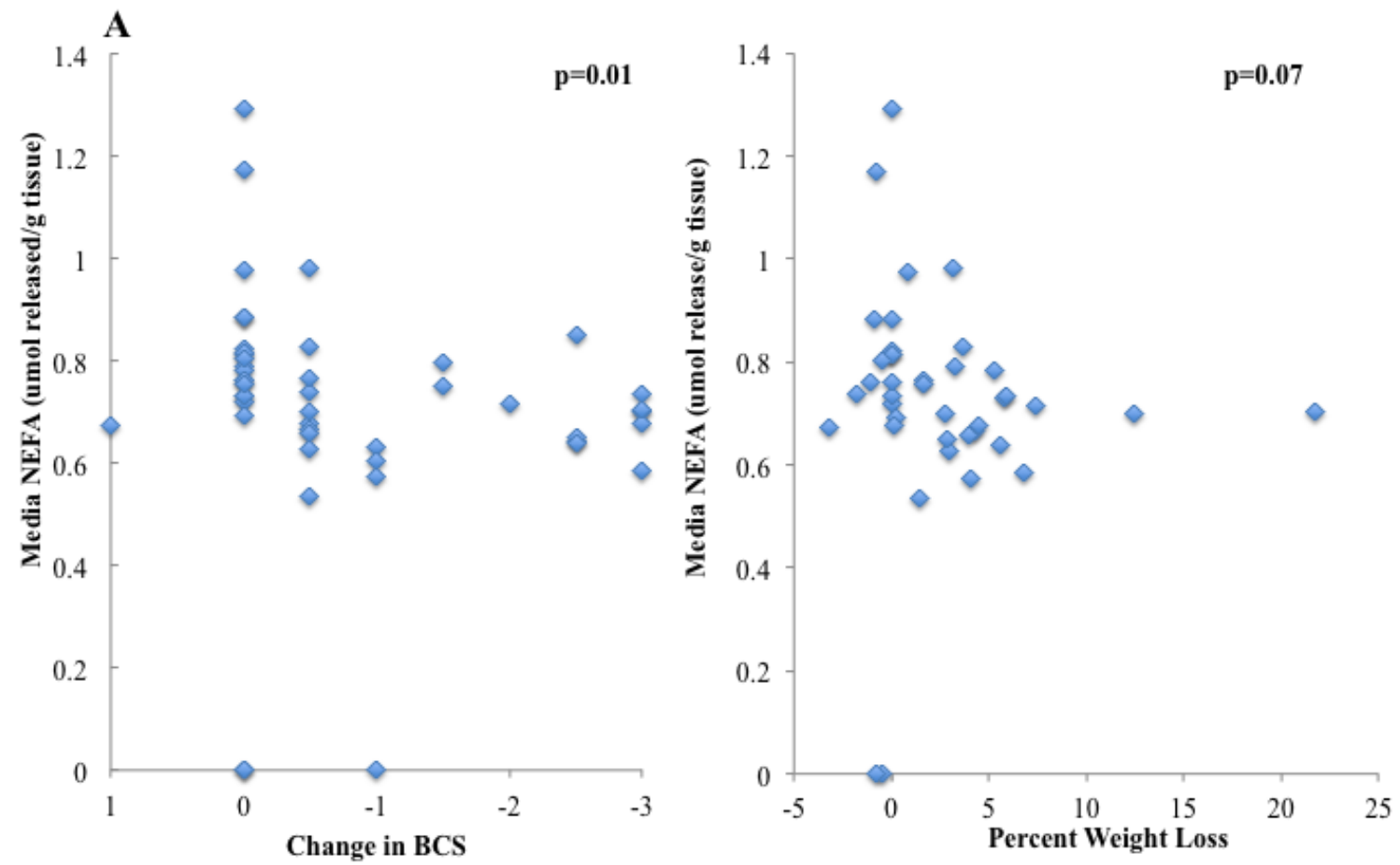
513 Figure 2 A-B: Influence of weight loss on lipolysis markers. Horses $(\mathrm{n}=8)$ were 514 enrolled in a baseline-sampling period lasting from $\mathrm{d} 0$ to $\mathrm{d} 14$, followed by a caloric 515 restriction from $\mathrm{d} 15$ to $\mathrm{d} 98$, where from $\mathrm{d} 15$ to $\mathrm{d} 43$ horses received a $30 \%$ digestible 516 energy reduction, and from $\mathrm{d} 44$ to $\mathrm{d} 98$ horses received a $30 \%$ restriction from 517 maintenance digestible energy. On d 0, 14, 28, 42, 70, and 98, a neck fat biopsy was 518 collected and immediately allocated for an ex vivo lipolysis assay. Media collected from 519 the assay was analyzed for non-esterified fatty acids (NEFA) and free glycerol 520 concentrations. The main effect of change in body condition score (BCS) and percent 521 weight loss were analyzed for each variable, with data points representing values at 522 corresponding points of BCS change or weight loss, respectively.
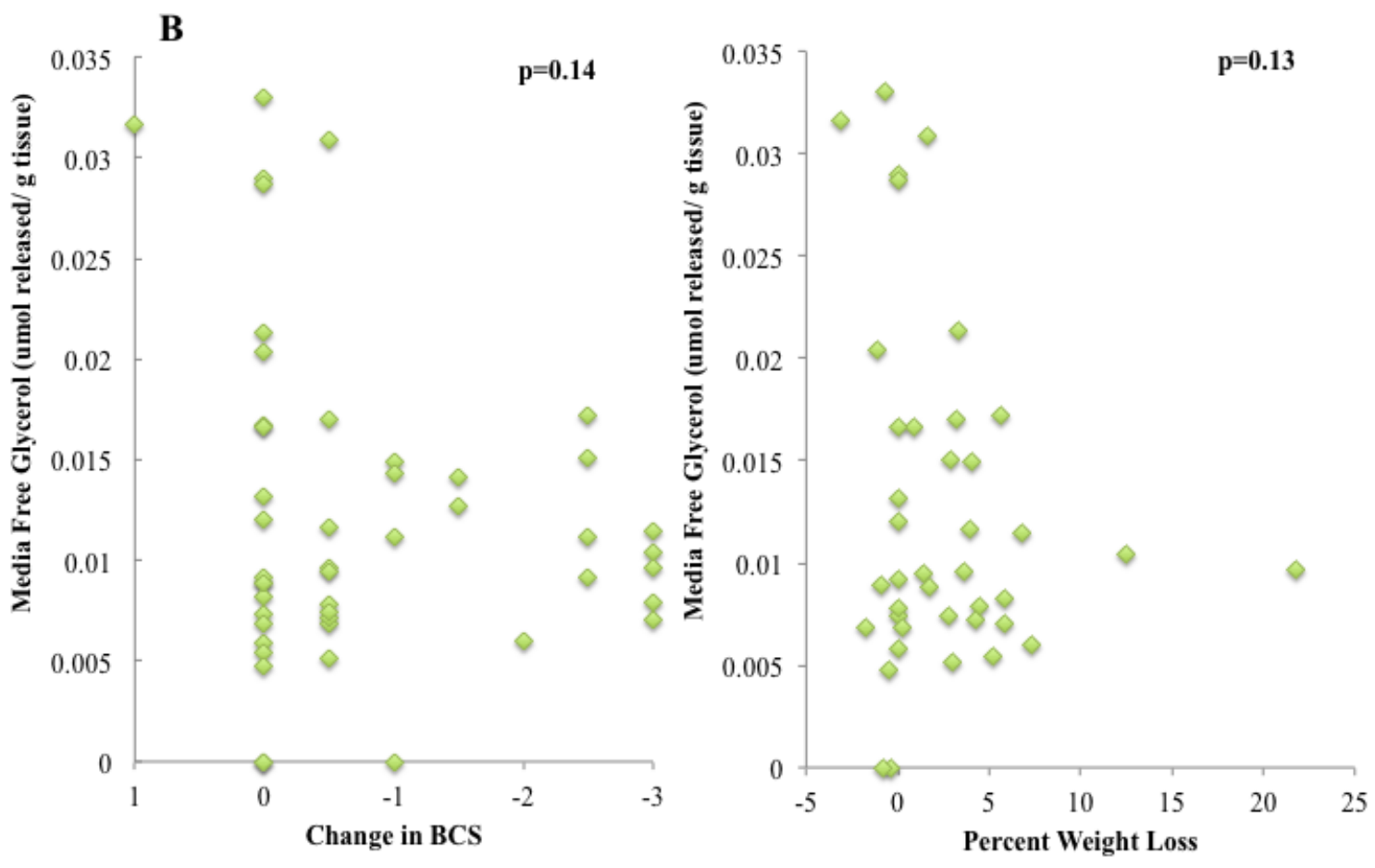
525 Figure 3 A-B: Glucose and insulin curves during baseline and end weight loss FSIGT.

526 Horses $(\mathrm{n}=8)$ were enrolled in a baseline-sampling period lasting from d0 to d14,

527 followed by a caloric restriction from $\mathrm{d} 15$ to $\mathrm{d} 98$, where from $\mathrm{d} 15$ to $\mathrm{d} 43$ horses received

528 a $30 \%$ digestible energy reduction, and from d44 to d 98 horses received a $30 \%$

529 restriction from maintenance digestible energy. On d 12-13 for the baseline sampling

530 period and d 94-95 for the weight loss period a frequently sampled intravenous tolerance

531 test (FSIGT) was administered to each horse in accordance with published methods

532 (Hoffman et al., 2003). A glucose bolus ( $0.3 \mathrm{~g} / \mathrm{kg} \mathrm{BW}, 50 \%$ dextrose solution) was given

533 at time point 0 , followed by an exogenous insulin bolus ( $30 \mathrm{mIU} / \mathrm{kg} \mathrm{BW}$, Humulin R)

534 administered at minute 20 , indicated by the green arrows. Blood samples were taken at -

$53530,0,1,2,3,4,5,6,7,8,9,10,12,14,16,19,22,23,24,25,27,30,35,40,50,60,70$,

$53680,90,100,120,150$ and 180 min post glucose administration and were analyzed for

537 plasma glucose and serum insulin. Area under the curve was calculated using the

538 trapezoidal method. The main effect of change in body condition score (BCS) and

539 percent weight loss were analyzed for each variable, with data points representing values

540 at corresponding points of BCS change or weight loss, respectively.

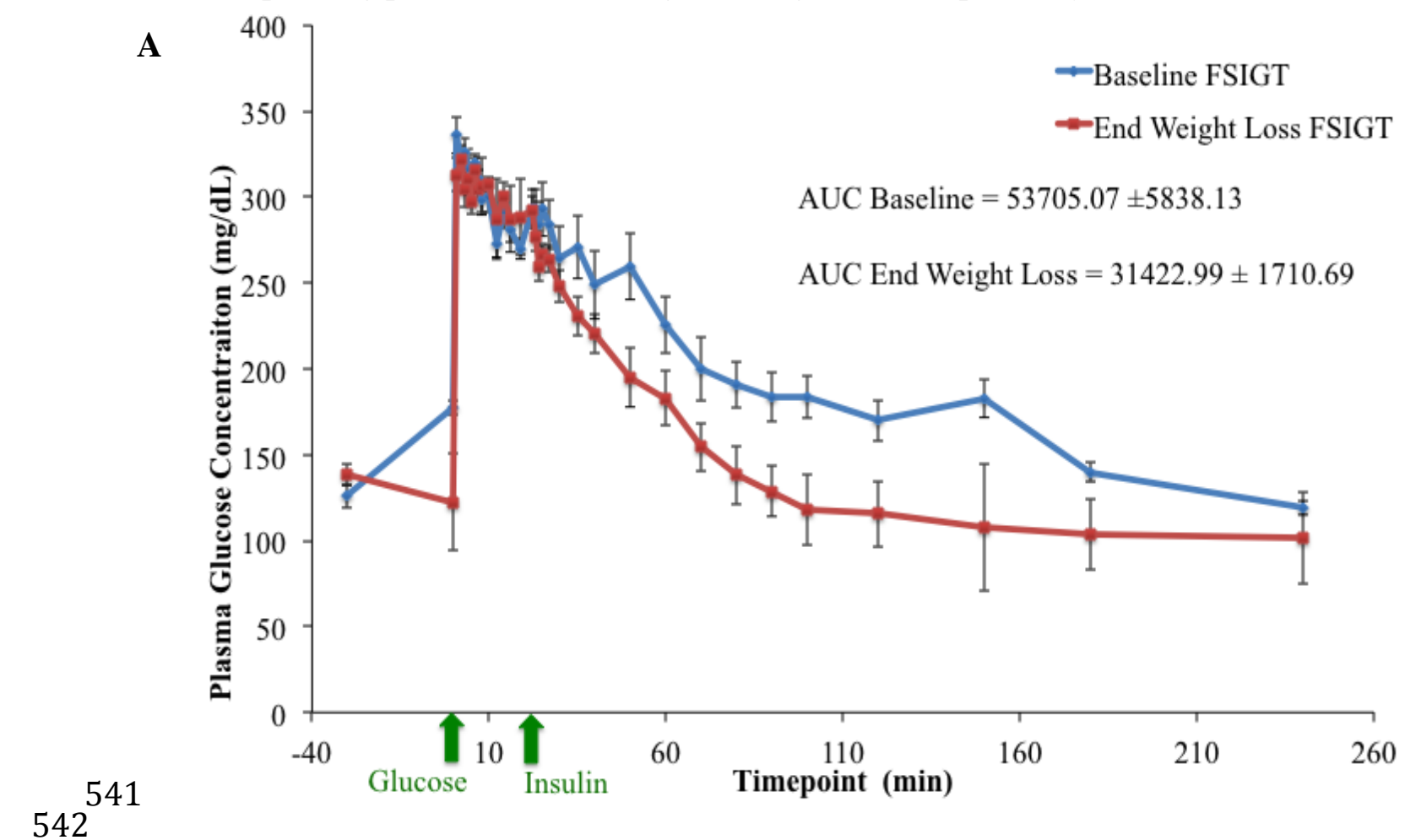


543 Figure 3 A-B: Glucose and insulin curves during baseline and end weight loss FSIGT.

544 Horses $(\mathrm{n}=8)$ were enrolled in a baseline-sampling period lasting from d0 to d14,

545 followed by a caloric restriction from $\mathrm{d} 15$ to $\mathrm{d} 98$, where from $\mathrm{d} 15$ to $\mathrm{d} 43$ horses received

546 a $30 \%$ digestible energy reduction, and from d44 to d 98 horses received a $30 \%$

547 restriction from maintenance digestible energy. On d 12-13 for the baseline sampling

548 period and d 94-95 for the weight loss period a frequently sampled intravenous tolerance

549 test (FSIGT) was administered to each horse in accordance with published methods

550 (Hoffman et al., 2003). A glucose bolus ( $0.3 \mathrm{~g} / \mathrm{kg} \mathrm{BW}, 50 \%$ dextrose solution) was given

551 at time point 0 , followed by an exogenous insulin bolus ( $30 \mathrm{mIU} / \mathrm{kg} \mathrm{BW}$, Humulin R)

552 administered at minute 20 , indicated by the green arrows. Blood samples were taken at -

$55330,0,1,2,3,4,5,6,7,8,9,10,12,14,16,19,22,23,24,25,27,30,35,40,50,60,70$,

$55480,90,100,120,150$ and 180 min post glucose administration and were analyzed for

555 plasma glucose and serum insulin. Area under the curve was calculated using the

556 trapezoidal method. The main effect of change in body condition score (BCS) and

557 percent weight loss were analyzed for each variable, with data points representing values

558 at corresponding points of BCS change or weight loss, respectively. 559

\section{0}

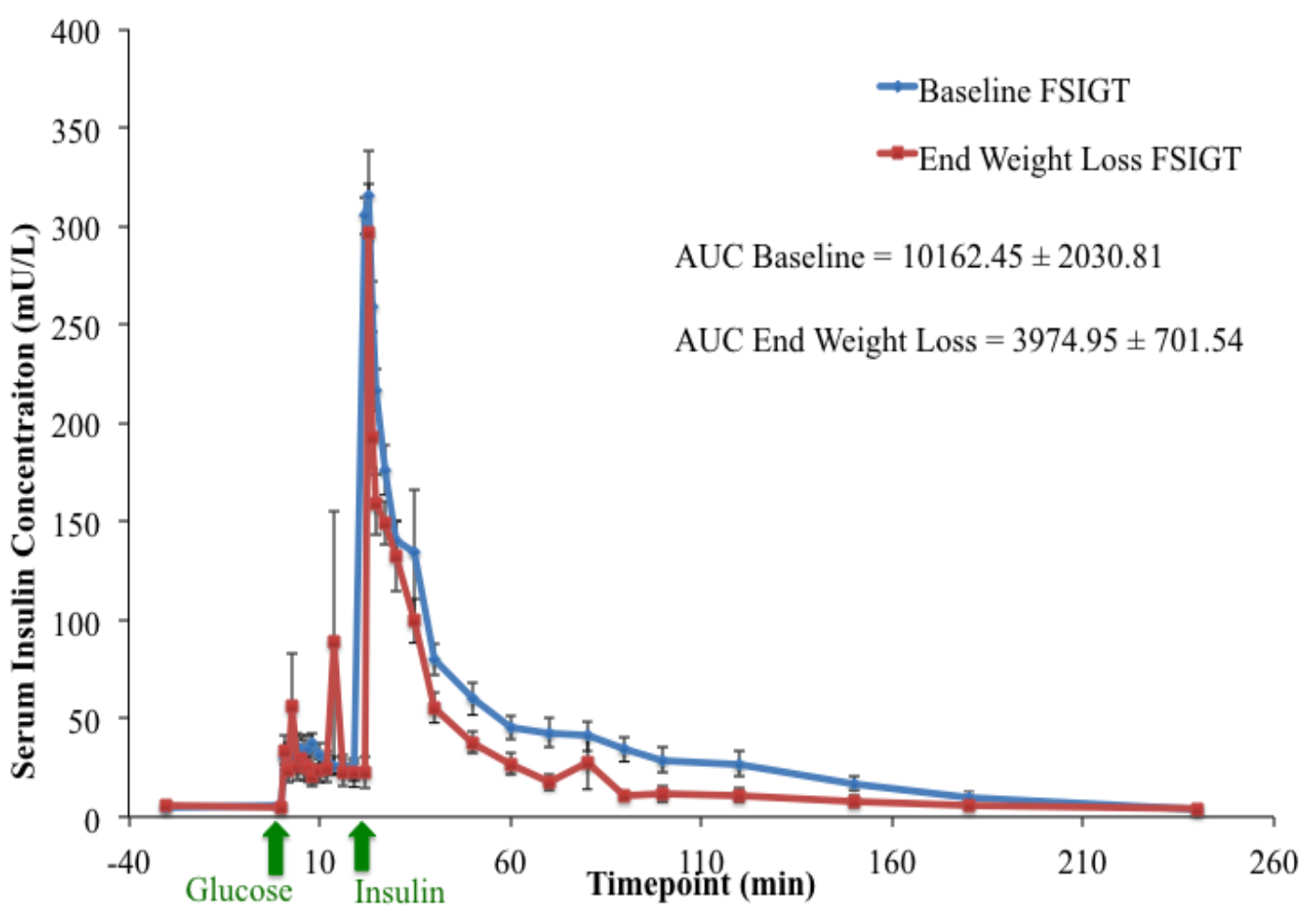

562

563

564 
565 Figure 4 A-B: Representative illustrations of glucose (A) and insulin (B) curves during 566 FSIGT by horse and date. Horses $(\mathrm{n}=8)$ were enrolled in a baseline-sampling period 567 lasting from $\mathrm{d} 0$ to $\mathrm{d} 14$, followed by a caloric restriction from $\mathrm{d} 15$ to $\mathrm{d} 98$, where from $\mathrm{d} 15$ 568 to $\mathrm{d} 43$ horses received a $30 \%$ digestible energy reduction, and from d44 to $\mathrm{d} 98$ horses 569 received a 30\% restriction from maintenance digestible energy. On d 12-13 for the 570 baseline sampling period (Date 1) and d 94-95 for the weight loss period (Date 2) a 571 frequently sampled intravenous tolerance test (FSIGT) was administered to each horse in 572 accordance with published methods (Hoffman et al., 2003). A glucose bolus ( $0.3 \mathrm{~g} / \mathrm{kg}$ 573 BW, 50\% dextrose solution) was given at time point 0 , followed by an exogenous insulin 574 bolus ( $30 \mathrm{mIU} / \mathrm{kg} \mathrm{BW}$, Humulin R) administered at minute 20. Blood samples were taken at $-30,0,1,2,3,4,5,6,7,8,9,10,12,14,16,19,22,23,24,25,27,30,35,40,50$, $60,70,80,90,100,120,150$ and 180 min post glucose administration and were analyzed for plasma glucose and serum insulin. Horses are numbered 1 through 8 , corresponding with the assignments described in table 1 , in order to illustrate independent horse variability during the study.

A

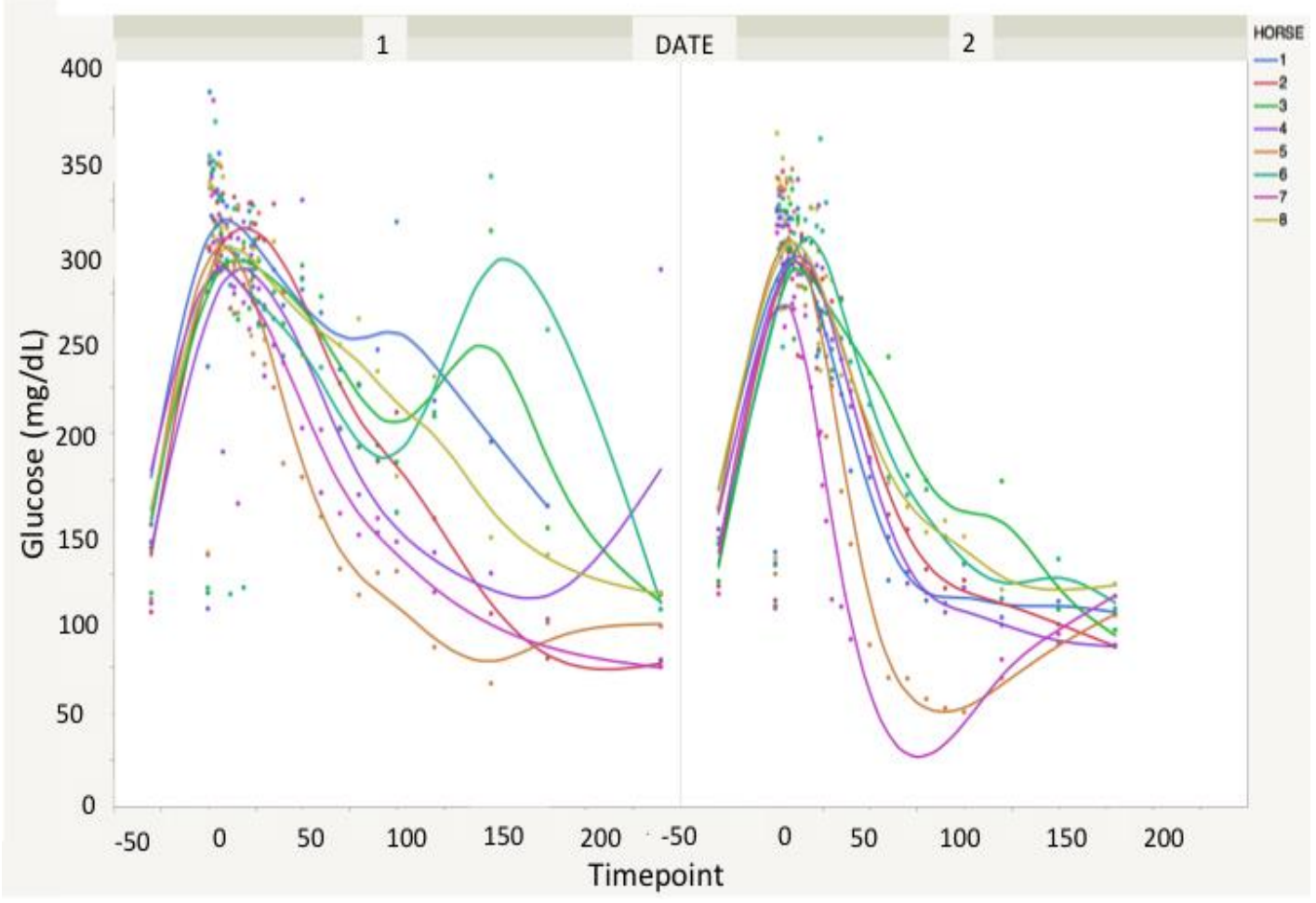


583 Figure 4 A-B: Representative illustrations of glucose (A) and insulin (B) curves during $584 \quad$ FSIGT by horse and date. Horses $(\mathrm{n}=8)$ were enrolled in a baseline-sampling period 585 lasting from $\mathrm{d} 0$ to $\mathrm{d} 14$, followed by a caloric restriction from $\mathrm{d} 15$ to $\mathrm{d} 98$, where from $\mathrm{d} 15$ 586 to $\mathrm{d} 43$ horses received a $30 \%$ digestible energy reduction, and from d44 to $\mathrm{d} 98$ horses 587 received a 30\% restriction from maintenance digestible energy. On d 12-13 for the 588 baseline sampling period (Date 1) and d 94-95 for the weight loss period (Date 2) a 589 frequently sampled intravenous tolerance test (FSIGT) was administered to each horse in 590 accordance with published methods (Hoffman et al., 2003). A glucose bolus $(0.3 \mathrm{~g} / \mathrm{kg}$ 591 BW, 50\% dextrose solution) was given at time point 0 , followed by an exogenous insulin 592 bolus (30 mIU/ kg BW, Humulin R) administered at minute 20. Blood samples were 593 taken at -30, 0, 1, 2, 3, 4, 5, 6, 7, 8, 9, 10, 12, 14, 16, 19, 22, 23, 24, 25, 27, 30, 35, 40, 50, $59460,70,80,90,100,120,150$ and 180 min post glucose administration and were analyzed 595 for plasma glucose and serum insulin. Horses are numbered 1 through 8 , corresponding 596 with the assignments described in table 1, in order to illustrate independent horse 597 variability during the study.

B

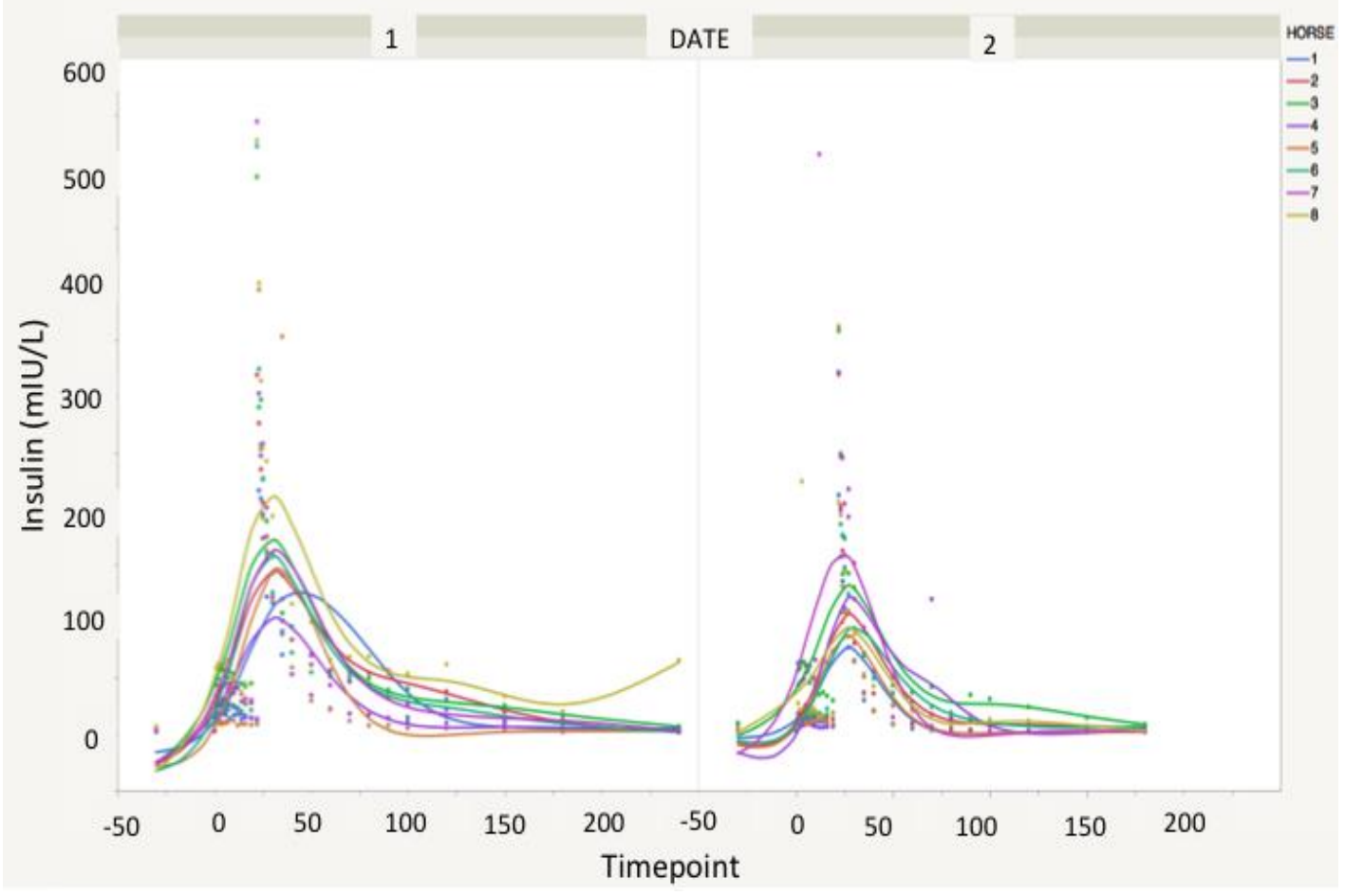

600

601 
602 Table 6: Minimal model parameters during baseline sampling and at the end of weight 603 loss.

604

\begin{tabular}{ccccc}
\hline \hline & & & \multicolumn{2}{c}{$p$ Value } \\
\cline { 3 - 5 } $\begin{array}{c}\text { Minimal Model } \\
\text { Parameters }\end{array}$ & $\begin{array}{c}\text { End of Baseline } \\
\text { Sampling } \\
\text { Period }^{2,4}\end{array}$ & $\begin{array}{c}\text { End of Weight } \\
\text { Loss Period }^{3,4}\end{array}$ & $\begin{array}{c}\text { BCS } \\
\text { Change }\end{array}$ & $\begin{array}{c}\text { Percent } \\
\text { Weight } \\
\text { Loss }\end{array}$ \\
\hline GB (mg/dL) & $159.9 \pm 28.7$ & $113.8 \pm 4.5$ & 0.07 & 0.003 \\
IB (mU/mL) & $4.51 \pm 0.80$ & $4.81 \pm 0.43$ & 0.53 & 0.96 \\
G0 (mg/dL) & $290 \pm 18$ & $329 \pm 20$ & 0.21 & 0.99 \\
Sg (min-1) & $0.0077 \pm 0.0023$ & $0.0234 \pm 0.0110$ & 0.41 & 0.81 \\
Si (x10-4 L*mU-1*min-1) & $1.078 \pm 0.270$ & $4.319 \pm 1.592$ & 0.04 & 0.01 \\
AIRg (mU*min*L-1) & $278.9 \pm 57.6$ & $217.0 \pm 61.2$ & 0.15 & 0.14 \\
DI & $260.17 \pm 72.6$ & $646.38 \pm 213.86$ & 0.19 & 0.09 \\
\hline
\end{tabular}

605

606

607

${ }^{1}$ Calculated from FSIGT

608 Sg refers to glucose sensitivity

609 Si refers to insulin sensitivity

610 AIRg refers to acute insulin response to glucose

611 DI refers to the disposition index

612 GB refers to calculated baseline glucose concentration

613 IB refers to calculated baseline insulin concentration

614 G0 refers to highest concentration of glucose after bolus intravenous injection

$615{ }^{2}$ Sampled on d12 or d13

$616{ }^{3}$ Sampled on d 95 or d 96

$617{ }^{4}$ Mean \pm SEM 
618 Table 7: Correlations between morphometric measurements, body composition, 619 circulating metabolic markers, and lipolysis data ${ }^{1}$

620

\begin{tabular}{|c|c|c|c|c|c|c|c|c|c|c|}
\hline \multirow{2}{*}{ 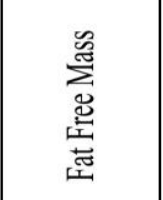 } & 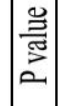 & $\begin{array}{l}\overline{8} \\
\dot{8}\end{array}$ & $\begin{array}{l}\overline{8} \\
\dot{8}\end{array}$ & 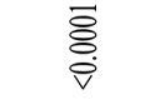 & $\begin{array}{l}\bar{\delta} \\
\dot{\sigma}\end{array}$ & $\begin{array}{l}\bar{\delta} \\
\dot{\nabla}\end{array}$ & $\vec{\sigma}$ & 年 & $\frac{2}{0}$ & తి \\
\hline & $\simeq$ & $\stackrel{\tilde{O}}{0}$ & రై & $\stackrel{e}{0}$ & $\stackrel{0}{0}$ & 롱 & $\stackrel{\infty}{0}$ & gे & $\overrightarrow{\widetilde{\delta}}$ & $\stackrel{\infty}{0}$ \\
\hline \multirow{2}{*}{ 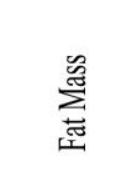 } & 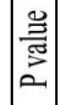 & ฮั. & $\cong$ & 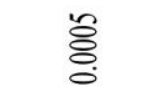 & శ్రి & ठ̊̊̊ & 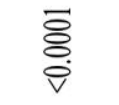 & 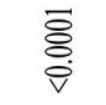 & 总 & 容 \\
\hline & $\simeq$ & $\tilde{3}$ & $\tilde{g}$ & $\stackrel{\infty}{0}$ & $\stackrel{\text { I }}{6}$ & 趈 & $\stackrel{\infty}{2}$ & కై & గి & $n$ \\
\hline \multirow{2}{*}{ 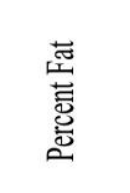 } & 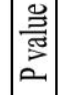 & శ్రి & $\tilde{3}$ & $\stackrel{0}{0}$ & ડิ & ह & 音 & 宊 & 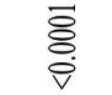 & 舫 \\
\hline & $\simeq$ & $\stackrel{7}{9}$ & $\stackrel{m}{9}$ & ס̊: & $\bar{\sigma}$ & 光 & f & $\sqrt[5]{2}$ & త్రం & 용 \\
\hline \multirow{2}{*}{ 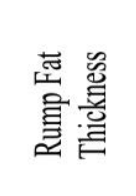 } & 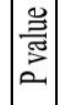 & f̊ & $\vec{\sigma}$ & ळం & g̊ & 3 & 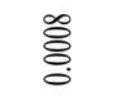 & 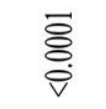 & 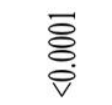 & 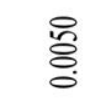 \\
\hline & $\simeq$ & בี & $\stackrel{0}{a}$ & $\tilde{\delta}$ & $\dot{0}$ & 5) & $\stackrel{\infty}{0}$ & ถి & B্ণ & 웡 \\
\hline \multirow{2}{*}{ 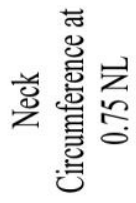 } & 善 & ఫ్రి & 훙 & 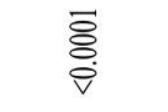 & $\begin{array}{l}\overline{8} \\
\dot{8}\end{array}$ & . & 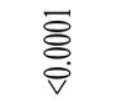 & $\frac{a}{0}$ & ڤ్ & హ。 \\
\hline & $\simeq$ & శี & \& & $\stackrel{0}{0}$ & $\ddot{0}$ & . & $\overline{\check{a}}$ & $\stackrel{9}{0}$ & $\stackrel{0}{\circ}$ & ?ొ \\
\hline \multirow{2}{*}{ 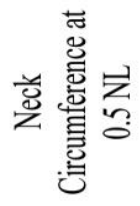 } & 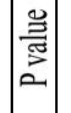 & 总 & 웅 & $\begin{array}{l}\bar{\delta} \\
\dot{\vec{\sigma}}\end{array}$ & . & . & 응 & o & హ. & ?ֶ. \\
\hline & $\simeq$ & gे & $\overline{3}$ & $\stackrel{尺}{\circ}$ & . & . & f & 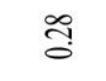 & 응 & f̊ㅁㅇ \\
\hline \multirow{2}{*}{ 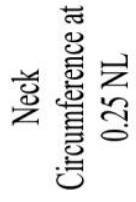 } & 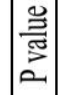 & 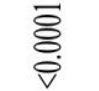 & 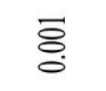 & . & . & . & ఠ̊ & ఫ్రి & gे & os \\
\hline & $\simeq$ & ర్రా & 守 & . & . & . & 志 & $\stackrel{\Xi}{0}$ & $\ddot{0}$ & $\tilde{\Omega}$ \\
\hline \multirow{3}{*}{ 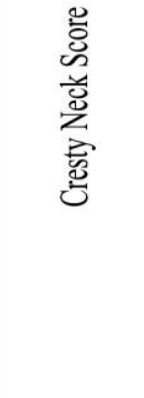 } & 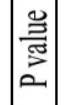 & శ్రి & $\stackrel{8}{0}$ & $\stackrel{\circ}{\circ}$ & ડ่ & గ్ & 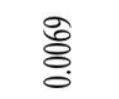 & 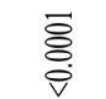 & . & రి \\
\hline & $\simeq$ & $\frac{m}{9}$ & స్̣ & ఫ్ & $\frac{ \pm}{i}$ & 串 & t & E. & . & $\overline{3}$ \\
\hline & & 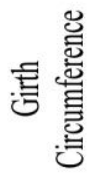 & 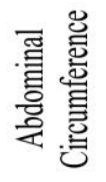 & 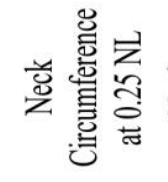 & 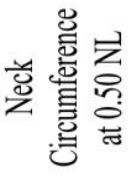 & 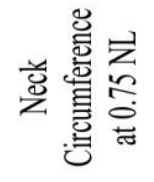 & 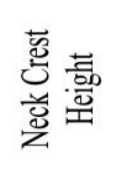 & 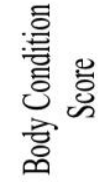 & 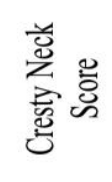 & 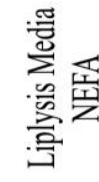 \\
\hline
\end{tabular}

621

$622{ }^{1} \mathrm{P}$ values reflect significance and trends identified with bonferroni corrections, where 623 significance $(*)$ determined at $\mathrm{p} \leq 0.0032$ and trends (\#) noted when $\mathrm{p} \leq 0.0047$. 


\section{Abstract}

628 Obesity causes a multitude of metabolic issues in the horse, yet stepwise alterations in

629 mitochondrial capacity and oxidant status during weight loss have yet to be analyzed.

630 Skeletal muscle contains subsarcolemmal (SSM) and interfibrillar (IFM) mitochondria,

631 which respond differently to physiological stimuli, impacting tissue and whole body

632 oxidant status. We hypothesized that 8 light-type horses would display improvements in

633 skeletal muscle mitochondrial subpopulation function and decreases in circulating

634 oxidant status markers during weight loss from an obese (7 to 8) to moderate (5) body

635 condition score (BCS). Effects of change in BCS or percent weight loss were evaluated

636 using the mixed procedure of SAS with repeated measures. Plasma nitrate decreased in

637 response to $B C S$ reduction $(p=0.06)$ and percent weight loss $(p=0.06)$, whereas

638 erythrocyte reduced glutathione $(\mathrm{p}=0.06)$ concentration increased with decreasing BCS.

639 Mitochondrial electron transport chain complex I and IV displayed greater activity in

640 SSM than IFM ( $\leq \leq 0.05)$, while I, III, and IV in SSM had decreased activity due to BCS

641 change and percent weight loss $(\mathrm{p} \leq 0.05)$. Interactions between SSM and IFM complex IV

642 activity and both weight loss markers $(\mathrm{p}<0.05)$ were displayed. Citrate synthase activity,

643 indicating mitochondrial number, was greater in SSM than IFM $(\mathrm{p}<0.0001)$ but remained

644 unchanged with weight loss parameters. Lipid peroxidation was decreased due to BCS

645 change $(\mathrm{p}=0.01)$ and weight loss $(\mathrm{p}=0.02)$, with greater concentration in SSM, but a

646 larger decrease in IFM $(\mathrm{p} \leq 0.05)$. Alteration in complex activities and lipid peroxidation 
647 suggest IFM are more affected by weight loss, with large contributions from complex IV

648 byproducts. Mitochondrial component flexibility may contribute individually to

649 development and disease propagation along with athletic performance of the equine

650 athlete.

651

652 Key Words

653 Mitochondria, horse, weight loss, oxidant status, metabolism

654

\section{Introduction}

656 Within the United States, obesity among horses has reached an all time high, where

657 nearly half of the nations horses are considered overweight or obese (Pleasant et al.,

658 2008). Obesity-related metabolic changes are well known within the horse, including

659 impairment of glucose tolerance and insulin signaling, which compounded with

660 compromised lipid metabolism and mitochondrial function can cause increases in

661 oxidative damage (Geor et al., 2007; Pleasant et al., 2008). While the effects of obesity

662 on physiologic pathways have been studied, many areas still remain vague, especially

663 with regard to the lasting effects of obesity on metabolic function and stepwise alterations

664 in metabolic capacity during weight loss. Within the muscle, mitochondrial

665 subpopulations may be differentially affected, but any impairment in function may give

666 rise to increased reactive oxygen species through leaky electron transport chain

667 complexes (Dabkowski et al., 2010). Elevated levels of oxidative damage can further

668 propagate the aforementioned dysfunction associated with obesity (Muoio and Newgard,

669 2006). Due to these and other effects, weight loss is often the desired goal, ideally 
670 translating a reduction in body condition into reversal of the impaired functions.

671 However, most studies in which effects of obesity have been evaluated utilized two

672 comparison groups; a lean or moderate and obese body condition models as opposed to

673 evaluating the effects of weight loss within a specific individual. Therefore, this study

674 was conducted to quantify the degree of oxidative damage and anti-oxidant defense along

675 with identifying functional differences in mitochondrial subpopulations of skeletal

676 muscle relative to body condition change and weight loss. We hypothesized that weight

677 loss would decrease circulating levels of nitric oxide, while increasing glutathione and

678 glutathione peroxidase levels. At the tissue level, lipid peroxidation would decrease

679 corresponding to a decrease in body condition. Further, we suspected that

680 subsarcolemmal mitochondria would show an increase in function, while interfibrillar

681 mitochondria would remain relatively unchanged.

682

683 Materials and Methods

684 The Middle Tennessee State University Institutional Animal Care and Use Committee

685 approved all methods and procedures used in this experiment. Horses were body

686 condition scored by two individual, experienced reviewers and horses with assigned an

687 body condition score (BCS) of 7 or greater (Henneke et al., 1983), were admitted to the

688 study. In addition, horses chosen for this screening process were known to not have any

689 predisposing metabolic conditions including Cushing's Disease or Metabolic Syndrome.

690 Eight, healthy, mature (5-19 yrs) light type mixed sex ( $\mathrm{n}=6$ mares, $\mathrm{n}=2$ geldings) horses

691 of Quarter Horse, Tennessee Walking Horse, or Standardbred breeds from the Middle 
692 Tennessee State University Horse Center herd were admitted for use in this study (Table

$6931)$.

694

695 Sampling Periods and Diet

696 Prior to and during the study, all horses received the same type of mixed grass hay and

697 commercial concentrate (Purina Strategy, Land O’Lakes Purina Mills, St. Louis, MO),

698 with ad libitum access to water. Dietary analysis was measured on concentrate samples

699 from 10 different bags and hay grab samples from at least 25 bales over 5 random dates

700 during the study (Equi-analyticial, Ithaca, NY, table 2 and 3). Further, study horses were

701 group housed in pastures with run-in shelters when not individually stalled over the entire

702 study. Pastures did contain some plant life; however, there was not enough coverage to

703 make a significant contribution to the diet and therefore was not included in DE

704 calculations or dietary analysis. Due to limiting labor factors, individual feed offerings

705 and refusals were not measured daily, but estimated based on average flake weight and

706 concentrate cup weight, taken from 10 total measurements.

707

708 The baseline sampling period (BSP) began on $\mathrm{d} 0$ and continued until $\mathrm{d} 14$, during which

709 time horses were maintained on their current dietary intake, with a mean DE intake of

$71021.1 \mathrm{Mcal} / \mathrm{d}$. Upon entry into the weight loss period (WLP, d15), horses received a 30\%

711 DE reduction by altering amount of concentrate and hay offered. Horses remained on this

712 level of restriction until d43, at which time calculations were made for maintenance DE

713 requirements based on current body weight, as described by the equation (NRC, 2007)

$714 \mathrm{DE}(\mathrm{Mcal} / \mathrm{d})=1.4+(0.03 \times \mathrm{BW})$. In order to facilitate further weight loss, a $30 \%$ 
715 reduction from the maintenance requirement was calculated and implemented throughout

716 the remainder of the study (mean DE intake 14.01). At no point during the study did

717 horses consume less than $1.5 \% \mathrm{BW}$ in forage per day to ensure proper hindgut health.

718

719 During both the BSP and WLP, all horses were subjected to exercise not exceeding the

720 parameters of light workload as defined by the NRC. Horses were exercised on average

721 between 1-3 hr/wk, with an approximate gait breakdown of $40 \%$ walk, $50 \%$ trot and $10 \%$

722 canter. Horses not broke to ride were lunged or worked in a round pen.

723

724 Sample Acquisition and Analysis

725 Morphometric Measurements and Body Condition Score

726 Two experienced individuals assigned a body condition score (BCS) based on a 1-9 scale

727 (Henneke et al., 1983). Scores were assigned on a whole or half score basis and the

728 average was used for statistical analysis. Morphometric measurements consisted of body

729 weight, wither height, body length, girth circumference, and abdominal circumference, in

730 accordance with the measurements made by Carter and colleagues (Carter et al., 2009).

731 Measurements and BCS were made on d 0, 14, 28, 42, 70, and 98. Body length was

732 measured from the intermediate tubercale of the humerous (point of the shoulder) to the

733 ischiatic tuberosity (point of the buttock). Girth circumference was measured

734 immediately behind the slope of the wither, caudal to the elbow (olecranon tuber).

735 Abdominal circumference was taken at two-thirds the distance from the intermediate

736 tubercale of the humerous (point of the shoulder) the point of the hip (tuber coxae). 
737 Horses were clipped in the areas where measurements were made initially to ensure later

738 measurements were taken at the same location.

\section{Body Composition Measurements}

741 Rump fat thickness was measured using B-mode ultrasound (Sonovet 2000, 5mgHz)

742 (Kearns et al., 2006a; Kearns et al., 2006b) on d 0, 14, 28, 42, 70, and 98. The site for

743 measurement was determined by measuring half the distance from the point of the hip

744 (tuber coxae) to the point of the buttock (ischiatic tuberosity); and half of the distance

745 from this point to the spine. Scans were made on alternating sides so as not to be affected

746 from healing biopsy sites. Body fat percentage was estimated using the following

747 equation (Kane et al., 1987); Percent Fat $=2.47+5.47$ (rump fat in $\mathrm{cm}$ ). Fat mass was

748 calculated by multiplying percent fat and total body mass. Fat free mass was determined

749 by the difference between total body mass and fat mass.

750

751 Circulating Oxidant Status Biomarkers

752 Non-fasting blood samples $(\sim 35 \mathrm{~mL})$ were collected via jugular venipuncture on d 0,14 ,

$75321,28,35,42,56,70$, and 98, placed into sodium heparin and EDTA vacutainers

754 (Vacutainer, Franklin Lakes, NJ). Tubes were immediately placed on ice. The EDTA

755 tube was centrifuged at $3000 \mathrm{x}$ g for $15 \mathrm{~min}$, while the sodium heparin tube was kept

756 aside and used for erythrocyte isolation. Plasma aliquots from the EDTA tube were

757 removed and stored at $-20^{\circ} \mathrm{C}$ until analysis.

758 
759 Sodium heparin plasma aliquots were used for nitric oxide approximation via the

760 measurement of total nitrate by a colorimetric kit (Nitric Oxide Kit, Bioassay Systems,

761 Hayward, CA) including deproteination for all plasma samples following the kit

762 instructions. Duplicate coefficient of variation was accepted as $10 \%$ or below. 763

764 Erythrocytes were isolated following a protocol adapted from Lamprecht and Williams 765 (Lamprecht and Williams, 2012). Briefly, $500 \mu \mathrm{L}$ whole blood from a sodium heparin

766 tube was transferred into a microcentrifuge tube, spun at $2500 \mathrm{xg}$ for $5 \mathrm{~min}$ at $4^{\circ} \mathrm{C}$, and

767 the plasma supernatant was removed. Erythrocytes were then washed with $500 \mu \mathrm{L}$ of

$7680.9 \%$ sodium chloride solution and centrifuged again at the same speed, duration and

769 temperature. The supernatant was discarded and $1 \mathrm{~mL}$ ice-cold distilled deionized water

770 was added to lyse the cells. Erythrocyte lysate was stored at $-80^{\circ} \mathrm{C}$ until analysis for

771 reduced glutathione (GSSH) and glutathione peroxidase-1 (GPx-1). Both GSSH and

772 GPx-1 were analyzed using commercially available kits (GSH-400 and GPx-340, Oxis

773 Biomedical Research, Foster City, CA). Protocols on the kit insert were followed and

774 sample volumes adjusted to produce repeatable results within the detectable range. The

775 accepted coefficient of variation between duplicates was $10 \%$ for both assays.

776

$777 \quad$ Muscle Biopsies

778 Muscle biopsies were collected on d 0, 14, 28, 42, 70, and 98. Prior to biopsy sampling,

779 biopsy sites were measured, clipped, and scrubbed with chlorhexadine solution followed

780 by an isopropyl alcohol rinse. Horses were then lightly sedated with xylazine $(0.5 \mathrm{mg} / \mathrm{kg}$ 
$781 \mathrm{BW}$ ) and were administered $10 \mathrm{cc}$ lidocaine anesthesia to the rump site. Consecutive

782 samples were taken on alternating sides so as to allow sites to fully heal.

784 Muscle biopsy site measurements were calculated by determining half the distance from

785 the point of the hip (tuber coxae) to the point of the buttock (ischiatic tuberosity). From

786 this point, a measurement was made to the spine, and biopsy site was placed at half this

787 length, with preparations made as stated above (Andrews et al., 1993). A Bergstrom

788 biopsy needle (6mm diameter, $10 \mathrm{~cm}$ total length) was used to access the middle gluteal

789 muscle (Kline et al., 1987; Liburt et al., 2012), where $1 \mathrm{~g}$ of tissue was removed. Biopsy

790 sites were stapled closed, and left to heal for $10 \mathrm{~d}$. Weighed sample allocations were

791 made for immediate mitochondrial isolation.

792

793 Mitochondrial Subpopulation Isolation

794 Muscle biopsies were blotted dry, weighed into $100 \mathrm{mg}$ pieces and rinsed with ice-cold

795 Chappel-Perry buffer in preparation for SSM and IFM isolation. Methods were followed

796 in accordance to those used by Palmer and colleagues (Palmer et al., 1977b) with minor

797 modifications (Dabkowski et al., 2010; Dabkowski et al., 2009; Dabkowski et al., 2008b).

798 Briefly, the samples were homogenized 1:10 (wt/vol) in ice-cold Chappel-Perry buffer,

799 containing (in mmol/L) $100 \mathrm{KCl}, 50 \mathrm{MOPS}, 5 \mathrm{MgSO}_{4} \cdot 7 \mathrm{H}_{2} \mathrm{O}, 1 \mathrm{EGTA}$, and $1 \mathrm{ATP}$ at pH

800 7.4. Homogenates were centrifuged at $700 \mathrm{x}$ g for $10 \mathrm{~min}$ and supernatant containing

801 SSM was removed and centrifuged again at $10,000 \mathrm{x} \mathrm{g}$ for $10 \mathrm{~min}$ to yield the SSM

802 fraction. A series of rinse and centrifugation at $10,000 \mathrm{x} \mathrm{g}$ for $10 \mathrm{~min}$ were conducted to

803 obtain a clean SSM fraction, resuspended in $100 \mathrm{uL} \mathrm{KCl-MOPS-EGTA} \mathrm{buffer.} \mathrm{The}$ 
804 remaining pellet from the $700 \mathrm{x}$ g spin was resuspended in KCl-MOPS-EGTA buffer

805 containing, in mmol/L, $100 \mathrm{KCl}, 50 \mathrm{MOPS}$ and $0.5 \mathrm{EGTA}$ at $\mathrm{pH} 7.4$ and exposed to 3.75

$806 \mathrm{mg} / \mathrm{g}$ trypsin for $7 \mathrm{~min}$. After $7 \mathrm{~min}$, the resuspended IFM were diluted twofold with

807 buffer plus protease inhibitor cocktail (Biovision, Mountain View, CA) to inhibit further

808 trypsin activity and centrifuged at $700 \mathrm{x} \mathrm{g}$ for $10 \mathrm{~min}$. The IFM-supernatant was saved

809 while the remaining pellet was resuspended in KCl-MOPS-EGTA buffer and spun down

810 again at $700 \mathrm{xg}$ for $10 \mathrm{~min}$ to maximize IFM yield. The IFM supernatants were

811 combined and spun down at 10,000 x g for $10 \mathrm{~min}$. After a series of wash steps, IFM

812 were spun down at $10,000 \mathrm{x}$ g for $10 \mathrm{~min}$ and the resulting pellet was resuspended in 100

813 uL KCl-MOPS-EGTA buffer. Both populations were stored at $-80^{\circ} \mathrm{C}$ until analysis.

814

815 Complex Activities and ATP Synthase

816 Electron transport chain complexes I, III, and IV activities were measured

817 spectrophotometrically (Biotek Synergy HT plate reader, Biotek, Winooski, VT) on

818 isolated frozen-thawed mitochondria as described by Dabkowski and colleagues

819 (Dabkowski et al., 2010). Complex I activity was measured by the oxidation of NADH in

820 the presence of an assay mixture containing $25 \mathrm{mM}$ potassium phosphate buffer ( $\mathrm{pH}$ 7.2),

$8215 \mathrm{mM} \mathrm{MgCl}_{2}, 2 \mathrm{mM} \mathrm{KCN}, 2.5 \mathrm{mg} / \mathrm{mL}$ bovine serum albumin, $50 \mu \mathrm{M} \mathrm{NADH}, 10 \mu \mathrm{M}$

822 decylubiquinone, and $2 \mu \mathrm{g} / \mathrm{mL}$ antimycin $\mathrm{A}$ at $340 \mathrm{~nm}$. The reaction was initiated at the

823 addition of isolated skeletal muscle mitochondrial subpopulations, and enzyme activity

824 was measured every $10 \mathrm{~s}$ for $3 \mathrm{~min}$. Complex I specific activity was inhibited by $2 \mu \mathrm{g} / \mathrm{mL}$

825 rotenone. Complex III activity was quantified by measuring the reduction of cytochrome

$826 \mathrm{c}$ at $550 \mathrm{~nm}$. An assay buffer containing $2 \mathrm{mM}$ EDTA, $500 \mathrm{mM}$ sucrose, $100 \mathrm{mM}$ 
827 Tris $\cdot \mathrm{HCl}(\mathrm{pH} 7.4), 1 \mathrm{mM}$ cytochrome c, $1 \mathrm{mg} / \mathrm{mL}$ antimycin $\mathrm{A}$ and reduced

828 decycloubiquinone was combined with mitochondrial isolations and spectrophotometric

829 measurements were made in $10 \mathrm{~s}$ increments for $3 \mathrm{~min}$. Lastly, complex IV was measured

830 by adding an assay mixture of $10 \mathrm{mM}$ phosphate buffer (pH 7.4) and $20 \mu \mathrm{M}$ reduced

831 cytochrome c, where readings at $550 \mathrm{~nm}$ every $10 \mathrm{~s}$ for $3 \mathrm{~min}$ began at the addition of

832 mitochondrial isolate determined oxidation of cytochrome c. Protein content was

833 determined via the Bradford assay (Bradford, 1976) using bovine serum albumin as the

834 standard. Values for complex activity data are expressed as nmol substrate consumed per

835 min per mg protein.

836

837 ATP synthase activity was measured via approximation of maximal hydrolytic capacity

838 of the enzyme in accordance with previously published methods (Dabkowski et al., 2010;

839 Feniouk et al., 2007; Pullman et al., 1960; Rosca et al., 2009). Activity was measured

840 spectrophotometrically via a coupled assay with lactate dehydrogenase and pyruvate

841 kinase as coupling enzymes on frozen-thawed mitochondria as oligomycin-sensitive

842 ATP-ase activity (Barrientos, 2002). Briefly, the mitochondria were incubated with a

843 buffer containing, in mmol/L, 20 HEPES, $5 \mathrm{MgCl} 2,100 \mathrm{KCl}, 2.5$ phosphoenolpyruvate,

844 and $0.2 \mathrm{NADH}$, along with $0.1 \mathrm{mg} / \mathrm{mL}$ pyruvate kinase and $0.1 \mathrm{mg} / \mathrm{mL}$ lactate

845 dehydrogenase, at combined $\mathrm{pH}$ of 7.5-8.0. The reaction was initiated by adding ATP to

846 a final concentration of $1 \mathrm{mM}$ and followed by NADH reduction at $340 \mathrm{~nm}$. Absorbance

847 was monitored in $10 \mathrm{~s}$ increments for 3 min using a Biotek Synergy HT plate reader

848 (Biotek, Winooski, VT). Protein content was measured as described above, with data 
849 expressed as $\mu \mathrm{mol}$ consumed per min per mg protein, which is equal to nmol of $\mathrm{NADH}$

850 oxidized per min per mg of protein.

851

852 Citrate Synthase

853 Citrate synthase activity was measured on frozen-thawed mitochondria utilizing the

854 reaction 5', 5'-Dithiobis 2-nitrobenzoic acid (DTNB) and CoA-SH to form TNB, as

855 instructed by a commercially available kit (Citrate Synthase Activity, ScienCell Research

856 Labroatories, Carlsbad, CA) (Morgunov and Srere, 1998; Trounce et al., 1996). The

857 intensity of the absorbance of the assay at $412 \mathrm{~nm}$ is proportional to citrate synthase

858 activity in the sample. As the assay was not completed on a fresh isolation, citrate

859 synthase activity is used as a measure of total mitochondrial population in each isolation,

860 rather than cleanliness of the isolation procedure. Data are expressed as activity/mg

861 mitochondria. Mitochondrial content was measured by protein concentration assayed and

862 calculated as stated above.

863

$864 \quad$ Lipid Quantification and Peroxidation

865 In order to estimate total lipid presence in mitochondrial isolations, a sulfo-phospho-

866 vanillin total lipid quantification procedure was conduced in accordance to previously

867 published methods (Cheng et al., 2011). To summarize, each sample was diluted in a 2:1

$868(\mathrm{v} / \mathrm{v})$ chloroform:methanol mixture and allowed to fully evaporate. At this point, $100 \mu \mathrm{L}$

869 concentrated sulfuric acid was added to each well, incubated at $90^{\circ} \mathrm{C}$ for $20 \mathrm{~min}$, and

870 allowed to cool at room temperature before reading for background absorbance at $540 \mathrm{~nm}$

871 (Biotek Synergy HT plate reader, Biotek, Winooski, VT). Then, $50 \mu \mathrm{L}$ vanillin and 
872 phosphoric acid mixture $(0.2 \mathrm{mg} / \mathrm{mL}$ Vanillin in $17 \%$ phosphoric acid $)$ were then added

873 to each well and allowed to develop for 10 min before a final absorbance was read at the

$874540 \mathrm{~nm}$. The difference between the two readings was calculated and sample

875 concentrations were calculated from a standard curve in $\mu \mathrm{g} / \mu \mathrm{L}$.

876

877 Lipid peroxidation was assessed by the measurement of two stable end products, 878 malondialdehyde (MDA) and 4-hydroxyalkenal (4-HAE), formed from the oxidation of

879 polyunsaturated esters and fatty acids (Dabkowski et al., 2009; Dabkowski et al., 2008a).

880 Frozen-thawed mitochondrial subpopulation isolations were analyzed for MDA and 4-

881 HAE using a commercially available colorimetric kit (Oxford Biomedical Research,

882 Oxford, MI). In brief, this assay is conducted based upon the reaction of a chromogenic

883 reagent, $\mathrm{N}$-methyl-2-phenylindole, reagent 1, with MDA and 4-HAE at $45^{\circ} \mathrm{C}$. One

884 molecule of either MDA or 4-HAE reacts with one molecule of reagent 1 to yield a stable

885 chromophore, detectable at $586 \mathrm{~nm}$. Absorbance was measured on a Biotek Synergy HT

886 plate reader (Biotek, Winooski, VT), where protein concentration allowed for equal

887 sample loading. Final values are expressed per $\mu \mathrm{g} / \mu \mathrm{L}$ total mitochondrial lipid.

888

889 Statistical Analysis

890 Data are expressed as mean \pm SEM. Normality of all data were assessed prior to

891 statistical analysis (SAS Institue, V9.3, Cary, NC). Any variable found to be non-

892 normally distributed by evaluation of kurtosis and skewness values, along with

893 significance in tests for location (students t and sign tests) and normality (Shapiro-Wilk

894 and Klomogorov-Smirinov tests) where $\mathrm{p} \leq 0.05$, were $\log _{10}$ transformed. The effect of 
895 BCS change and percent weight loss was tested for all data using proc MIXED procedure 896 of SAS with repeated measures. Gender, horse, age and date were included in the class

897 statement during repeated measures analysis. Similarly, when evaluating complex I, III,

898 IV, ATP synthase, citrate synthase and activities, along with lipid peroxidation,

899 subpopulation was also included in the model and class. The model with the best fit

900 according to Akaike's Information Criterion used a compound symmetry structure. A

901 Pearsons Correlation was run using the proc CORR procedure of SAS, with Bonferroni

902 correction to prevent the incidence of type I errors when performing multiple

903 comparisons. For all data except correlations $\mathrm{p}<0.05$ was considered significant and

904 trends were noted at $\mathrm{p}<0.10$. Due to the bonferroni correction, $\mathrm{p}<0.0021$ was considered

905 significant and trends were noted when $\mathrm{p}<0.0052$ for all correlations.

906

907 Results

908 Body and Morphometric Measurements

909 Horses on the study transitioned from a mean BCS of $7.8 \pm 0.02$ a moderate body

910 condition, $5.0 \pm 0.0$ during the course of the weight loss period. A summary of horse age,

911 breed, sex and body characteristics are reported in table 1 . Averages of body weight, girth

912 circumference, abdominal circumference, RFT, percent fat, fat mass and fat free mass are

913 reported in table 4.

914

915 Circulating Oxidant Status Markers

916 In relationship to change in body condition score, trends toward significance were

917 displayed for decreasing nitrate concentration $(\mathrm{p}=0.06)$ and increasing reduced 
918 glutathione concentration ( $\mathrm{p}=0.07$ ), while no differences were noted with regard to GPx

919 (figure 1). When analyzed in response to percent weight loss, glutathione and GPx were

920 not significant but nitrate concentration retained a trend $(\mathrm{p}=0.06$, figure 1 A-C).

921

922 Electron Transport Chain Enzyme Activities, Citrate Synthase and Lipid Peroxidation

923 Significant differences were found between mitochondrial subpopulations regarding

924 decreasing complex I, and IV activity, while a trend was observed for complex III

925 activity, where $\mathrm{p}=0.02,0.001$, and 0.06 , respectively. Further, activity in complex I

$926(\mathrm{p}=0.01), \mathrm{III}(\mathrm{p}<0.0001)$ and IV $(\mathrm{p}=0.001)$ decreased in response to change in BCS. The

927 interaction between mitochondrial subpopulation and change in body condition score was

928 significant for complex IV ( $\mathrm{p}=0.001)$, but not complexes I or III where IFM decreased

929 with change in BCS but SSM did not. ATP synthase activity was not found to be

930 significantly affected for any comparison or interaction. Data analysis for complexes I, III

931 and IV in addition to ATP synthase are represented in figure 2 A-D.

932

933 When complex enzyme activities were analyzed with respect to percent weight loss,

934 complex I displayed a decrease in activity with regard to mitochondrial subpopulation

$935(\mathrm{p}=0.03)$ and percent weight loss $(\mathrm{p}=0.002)$, but no effect was denoted for the interaction

936 (Figure 2A). Complex III displayed a significant reduction in activity in response to

937 weight loss $(\mathrm{p}<0.0001)$, and a trend for differences between subpopulations $(\mathrm{p}=0.06)$ yet

938 the interaction was not significant (Figure 2B). Subpopulation ( $\mathrm{p}=0.003)$ and weight loss

$939(\mathrm{p}=0.01)$ caused a decrease in complex IV activity, further displayed by an interaction

940 between these parameters ( $\mathrm{p}=0.05$, Figure $2 \mathrm{C}$ ), signifying differential effects of weight 
941 loss parameters on each subpopulation within this complex. No differences were found

942 with regard to ATP synthase activity and any included model parameter (Figure 2D).

943

944 As an approximate measure of mitochondrial number, citrate synthase activity was found

945 to be different between subpopulations $(\mathrm{p}<0.0001)$ in both models where SSM showed a

946 higher concentration than IFM, but no effect of change in body condition score, percent

947 weight loss or appropriate interaction was observed (Figure 3).

948

949 A significant effect of subpopulation ( $\mathrm{p}=0.01)$ was observed where SSM displayed

950 greater amounts of lipid peroxidation than IFM. Change in body condition and

951 interactions between model components were observed for lipid peroxidation $(\mathrm{p}=0.01$,

9520.004 , and 0.05 , respectively) where peroxidation levels decreased due to reduction in

953 BCS. Similarly, when evaluated in response to weight loss, significance was observed

954 between higher levels of peroxidation in the SSM and IFM mitochondrial subpopulations

$955(\mathrm{p}=0.02)$, and decreases in peroxidation due to percent weight loss $(\mathrm{p}=0.01)$, with a trend

956 for significance displayed for the interaction ( $\mathrm{p}=0.07$ Figure 4$)$.

957

958 Correlations

959 Body condition score was positively correlated to lipid peroxidation in the IFM

960 ( $\mathrm{p}=0.002)$. Complex III activity in the SSM $(\mathrm{p}<0.0001)$ and complex IV $(\mathrm{p}=0.0002)$

961 activity in the IFM showed a significant positive correlation to BCS. Similarly when

962 correlating these variables to cresty neck score, significance was determined between 
963 SSM complex III activity $(\mathrm{p}=0.002)$ and a trend was noted between IFM complex IV

964 activity $(\mathrm{p}=0.003)$.

965

966 Citrate synthase activity in the SSM was negatively correlated to complex IV in the same

967 subpopulation $(\mathrm{p}=0.002)$. Further, citrate synthase activity between the subpopulations

968 displayed a significant positive correlation $(\mathrm{p}<0.0001)$. Interfibrillar citrate synthase

969 activity was significantly correlated to lipid peroxidation within the subpopulation

$970 \quad(\mathrm{p}<0.0001)$. A summary of these and other correlation data can be found in table 5.

971

\section{Discussion}

973 Body composition is an important factor to consider when evaluating metabolic function,

974 specifically with regard to glucose and lipid metabolism (Pedersen et al., 2003; Reaven,

975 1995). Due to the subjective nature of the Henneke body condition scoring system along

976 with wanting to examine changes in regional adiposity, we included morphometric

977 measurements to provide a comprehensive picture of phenotypic body adipose deposits

978 (Carter et al., 2009; Henneke et al., 1983); however, body composition can be

979 challenging to measure in a live animal. The concurrent reduction in rump fat thickness

980 with decreasing body weight suggests horses were loosing fat mass as they transitioned to

981 a moderate body condition. Further, the reduction in fat free mass suggests that some

982 portion of lean body tissues were utilized for energy homeostasis, consistent with

983 metabolic processes during calorie restriction. Although horses were enrolled in a light

984 exercise program during the time of the study, horses were acclimated to this level of

985 exercise; therefore, no skeletal muscle adaptation to workload was expected. 
987 Skeletal muscle mitochondrial distribution is directly related to fiber type composition

988 and therefore responses to physiological states can be altered depending on muscle fiber

989 profile. In humans, metabolic disturbances associated with obesity and diabetes are

990 associated with low percentages of type I fibers (Hickey et al., 1995). Further, mice

991 engineered to have a high number of type I fibers are more insulin sensitive and less

992 susceptible to diet-induced obesity (Ryder et al., 2003). The middle gluteal muscle

993 sampled during this study for mitochondrial evaluation reflects a mixed fiber type, where

994 previous research shows at a depth of $6 \mathrm{~cm}$ type I slow oxidative fibers represent $26.7 \pm$

$9955.9 \%$, type IIa fast oxidative fibers characterize $54.9 \pm 8.3 \%$ and type IIb fast glycolytic

996 fibers comprise $18.4 \pm 8.4 \%$, respectively (Kline et al., 1987). Therefore, we expect the

997 mitochondria isolated and analyzed in this study should reflect a mixed population and

998 role within functioning muscle. The distribution signifies an equal ability to tolerate both

999 aerobic and anaerobic respiration along with various energy substrates, thereby

1000 incorporating representative differences in weight loss across associated skeletal muscle

1001 fiber responses

1002

1003 Although it is recognized that skeletal muscle mitochondria exist together in a reticulum,

1004 compartmentalization into SSM and IFM allow for differential effects of physiologic

1005 intervention or disease states, and thus should be studied independently (Palmer et al.,

1006 1977a). For example, in mice subjected to streptozocin-induced type I diabetic insult,

1007 cardiomyocytes displayed greater IFM than SSM dysfunction, whereas in a db/db type 2

1008 diabetic model the SSM was more affected (Baseler et al., 2011; Dabkowski et al., 2010). 
1009 The SSM provide energy for membrane related processes including signal transduction,

1010 ion exchange, and substrate transport and substrate activation (Hood, 2001); metabolic

1011 processes necessary for insulin signaling and glucose metabolism. The SSM evaluated in

1012 this study did not display a large change in activity, and based on the relatively conserved

1013 function of the SSM complex activities during weight loss, it is plausible that the SSM

1014 played a role in the improved insulin sensitivity displayed in the aforementioned chapter.

1015 As the SSM were not dysfunctional during weight loss, their function may have been

1016 important to the modulation of the obese phenotype, reflected by the lack of change in

1017 glucose mediated glucose disposal and other minimal model parameters. Currently, the

1018 only available equine-related information has utilized activity of mitochondrial marker

1019 enzymes such as succinate dehydrogenase or mitochondrial volume density to estimate

1020 muscle oxidative capacity (Hoppeler et al., 1987; Kayar et al., 1989; Quiroz-Rothe and

1021 Rivero, 2001; Snow and Guy, 1980), with no data describing the roles of subpopulations

1022 on overall energy generation. In human and rodent obesity models, the SSM is primarily

1023 affected, displaying greater levels of complex activities during weight loss or when obese

1024 models are compared to a lean counterpart (Kelley et al., 2002a; Ritov et al., 2005;

1025 Toledo et al., 2007). Previous work from our laboratory suggests equine skeletal muscle

1026 mitochondria subpopulations may play a more equal role than that of rodents (Zambito et

1027 al., 2013). The greater malleability of IFM function with relationship to complex I, III

1028 and IV activity suggests that within the horse, this subpopulation may be more sensitive

1029 to physiological states.

1030 
1031 Further, results from this study display a decreasing maximal activity for complex I, III

1032 and IV in obese horses transitioning through weight loss. These results are contrary to

1033 most published research both relating to obesity-related, diabetic models, and weight loss

1034 influences on mitochondrial dysfunction in rodents and humans (Bajpeyi et al., 2011;

1035 Dabkowski et al., 2008a; Kelley et al., 2002b; Ritov et al., 2005; Toledo et al., 2007).

1036 Thus far, little work has been completed evaluating equine mitochondrial function

1037 (Votion et al., 2012) leaving many areas unclear with regard to species related differences

1038 in mitochondrial adaptation to physiological change. The higher level of activity when

1039 horses were at an obese body condition score may signify that the mitochondria

1040 compensated for excess dietary nutrient influx, and thus were the foundation for the lack

1041 of differences seen elsewhere. The flexibility of both populations of mitochondria,

1042 specifically the IFM, could not only be an evolutionary advantage for the horse to alter

1043 metabolic function in the face of differing nutritional status but also may tie into the

1044 inherent exercise capability of the horse over many other species.

1045

1046 In addition to their primary roles in substrate oxidation and ATP generation,

1047 mitochondria are also a primary source of reactive oxygen species. Declining complex

1048 activity should not necessarily be viewed as a negative result, as decreasing activity may

1049 reduce reactive oxygen species production. As body condition decreases and percent

1050 weight loss increases, nitrate levels as an indicator of nitric oxide decreases, while

1051 glutathione concentration increases. Combined these data suggest that overall whole body

1052 oxidant status was improving as horses lost weight. Glutathione peroxidase activity was 
1053 not altered due to weight loss, yet it is plausible that the level of oxidative stress was not

1054 elevated to the degree to impact enzyme activity.

1055

1056 Lipid peroxidation decreased in both SSM and IFM, with a greater level of damage

1057 observed in the SSM. As mentioned previously, electron transport chain complex I, III,

1058 and IV activity displayed a greater decrease in the IFM, thus generation of reactive

1059 species should decline in a similar manner. The resulting decrease in free radicals and

1060 reactive oxygen species would explain the decrease in lipid peroxidation observed in the

1061 IFM; a theory that could likewise be applied to the SSM. The higher level of lipid

1062 peroxidation observed in the SSM may be a function of mitochondrial number, as

1063 estimated by citrate synthase activity or position within the cell. Location directly

1064 beneath the plasma membrane may subject this population to oxidative insult from

1065 circulating oxidant species along with reactive oxygen species produced from the SSM

1066 alone.

1067

1068 Further, the possibility remains that due to high volume of oxidative phosphorylation

1069 capacity reflected by the nearly 50 -fold increase from resting to maximal $\mathrm{VO}_{2}$ (Poole,

1070 2004), horses may have adapted to tolerate higher levels of oxidative stress. Splenic

1071 contraction is a unique performance advantage to the horse (Poole, 2004) and even in an

1072 obese horse model and from an evolutionary perspective they still must retain a certain

1073 level of athletic ability despite a greater fat mass. The ability to tolerate high oxygen

1074 concentrations in the muscle without excessive oxidative damage may prove to be

1075 beneficial in the instance of obesity-induced increases in oxidant status. Further, if the 
1076 mitochondria had increased function to cope with an excess substrate load, the reduction

1077 in activity would signify return to a normal level of operation. Comparison of basal

1078 metabolic rates between mean obese and moderate BCS weights display a reduction from

$107918 \mathrm{Mcal} / \mathrm{d}$ to $16 \mathrm{Mcal} / \mathrm{d}$, as calculated by body weight $(\mathrm{kg})^{0.75}$ x $115 \mathrm{kcal}$. Decreased

1080 basal metabolic rate could also result in a lower complex activities and an overall

1081 reduction in oxidant status. Without supplemental data and analysis of the mechanisms

1082 behind mitochondrial function in the horse, exact understanding of these changes remains

1083 unanswered.

1084

1085 In conclusion, data suggest equine skeletal muscle mitochondrial subpopulations in this

1086 study contradict central dogma in human and rodent models regarding obesity- and

1087 weight loss-related influences on SSM and IFM function. The degree of obesity achieved

1088 prior to weight loss was adequate to induce increases in complex activity, and oxidant

1089 status within the body, yet not to the degree to transition into a metabolic disease state.

1090 Additional studies are warranted to not only elucidate mechanisms behind mitochondrial

1091 malleability in the equine model but also to fully determine the impact of mitochondrial

1092 function on exercise capacity.

1093

1094 Acknowledgements

1095 Funding for this project provided in part by the West Virginia University Summer

1096 Undergraduate Research Experience Program, Middle Tennessee State University, AHA

1097 13PRE16850066, NSF DGE1144676, and NIH DP2DK083095. Special recognition is

1098 warranted for the Middle Tennessee State University Horse Science Center employees, 
1099 including Jessica Shultz, Jeremy Carlson, Renee Dunn, Dr. John Haffner, the student 1100 farm workers, and the horse program graduate students for their assistance in the 1101 completion of this project.

1102 


\section{Tables and Figures}

1104 Table 1: Summary of individual horse characteristics

\begin{tabular}{ccccccc}
\hline \hline Horse & Age $(\mathrm{yr})$ & $\mathrm{Sex}^{1}$ & Breed & $\begin{array}{c}\text { Wither } \\
\text { Height } \\
(\mathrm{cm})^{2}\end{array}$ & $\begin{array}{c}\text { Body } \\
\text { Length } \\
(\mathrm{cm})^{3}\end{array}$ & $\begin{array}{c}\text { Neck } \\
\text { Length } \\
(\mathrm{cm})^{4}\end{array}$ \\
\hline 1 & 10 & $\mathrm{M}$ & Quarter Horse & 142.2 & 157.5 & 71.1 \\
2 & 6 & $\mathrm{M}$ & Tennessee Walker & 148.0 & 137.2 & 71.1 \\
3 & 13 & $\mathrm{M}$ & Standardbred & 160.0 & 156.2 & 101.6 \\
4 & 19 & $\mathrm{G}$ & Quarter Horse & 149.9 & 149.9 & 86.4 \\
5 & 5 & $\mathrm{M}$ & Quarter Horse & 147.3 & 152.4 & 81.3 \\
6 & 10 & $\mathrm{G}$ & Quarter Horse & 170.8 & 165.1 & 96.5 \\
7 & 19 & $\mathrm{M}$ & Quarter Horse & 151.8 & 158.8 & 95.3 \\
8 & 15 & $\mathrm{M}$ & Quarter Horse & 157.5 & 165.1 & 104.1 \\
\hline & & & Mean \pm SEM & $153.4 \pm 3.2$ & $155.3 \pm 3.3$ & $88.4 \pm 4.6$ \\
\hline
\end{tabular}

$1105{ }^{1}$ For sex categorization $\mathrm{M}$ represents mare and $\mathrm{G}$ represents gelding.

$1106{ }^{2}$ Measured from the ground to the highest point of the wither

$1107{ }^{3}$ Measured from the point of the shoulder to the point of the rump

$1108{ }^{4}$ Measured from the poll to the point of union between neck musculature and the wither

1109

1110

1111 
1112 Table 2: Nutrient analysis of grass hay and concentrate, as fed

\begin{tabular}{lcc}
\hline \hline Nutrient, as fed & Grass Hay & Concentrate \\
\hline Dry Matter (\%) & 95.8 & 93.4 \\
Digestable Energy (DE, Mcal/kg) & 1.85 & 2.84 \\
Crude Protein (g/kg) & 51.5 & 150.1 \\
Acid Detergent Fiber (ADF, g/kg) & 371.8 & 166 \\
Neutral Detergent Fiber (NDF, g/kg) & 625.8 & 330.8 \\
Water Soluble Carbohydrates (WSC, g/kg) & 56.0 & 89.4 \\
Ethanol Soluble Carbohydrates (ESC, g/kg) & 15.5 & 51.7 \\
Starch (g/kg) & 14.3 & 131.4 \\
Non-fiber Carbohydrates (NFC, g/kg) & 156.8 & 350.9 \\
Crude Fat (g/kg) & 28.6 & 59.3 \\
Calcium $(\mathrm{g} / \mathrm{kg})$ & 6.03 & 12.91 \\
Phosphorus $(\mathrm{g} / \mathrm{kg})$ & 0.83 & 8.15 \\
Sodium $(\mathrm{g} / \mathrm{kg})$ & 0.218 & 2.369 \\
\hline
\end{tabular}

1113

1114 Table 3: Percent composition of diet on a dry matter basis

\begin{tabular}{lcr}
\hline \hline Nutrient, \% dry matter & Grass Hay & Concentrate \\
\hline Crude Protein, \% & 5.4 & 16.1 \\
Acid Detergent Fiber, \% & 38.8 & 17.8 \\
Neutral Detergent Fiber, \% & 65.4 & 35.4 \\
Water Soluble Carbohydrates, \% & 5.9 & 9.6 \\
Ethanol Soluble Carbohydrates, \% & 1.6 & 5.5 \\
Starch, \% & 1.5 & 14.1 \\
Non-fiber Carbohydrates, \% & 16.4 & 37.6 \\
Crude Fat, \% & 3 & 6.3 \\
Calcium, \% & 0.63 & 1.38 \\
Phosphorus, \% & 0.09 & 0.87 \\
Sodium, \% & 0.023 & 0.254 \\
\hline
\end{tabular}

1115

1116

1117 
1118 Table 4: Physical descriptors during baseline sampling compared to end of weight loss

1119 period

\begin{tabular}{|c|c|c|c|c|}
\hline & \multicolumn{2}{|c|}{$\begin{array}{l}\text { Baseline Sampling } \\
\text { Period }^{1}\end{array}$} & \multicolumn{2}{|c|}{ End Weight Loss Period ${ }^{2}$} \\
\hline & Mean & SEM & Mean & SEM \\
\hline Weight $(\mathrm{kg})$ & 547.3 & 4.9 & 487.0 & 8.4 \\
\hline Body Condition Score ${ }^{3}$ & 7.8 & 0.02 & 5.0 & 0.00 \\
\hline Girth Circumference $(\mathrm{cm})^{4}$ & 194.9 & 0.6 & 188.3 & 1.2 \\
\hline Abdominal Circumference $(\mathrm{cm})^{5}$ & 213.6 & 0.7 & 199.7 & 1.9 \\
\hline Rump Fat Thickness (mm $)^{6}$ & 11.2 & 0.2 & 5.3 & 0.2 \\
\hline Percent $\mathrm{Fat}^{7}$ & 8.6 & 0.1 & 5.3 & 0.1 \\
\hline Fat Mass $(\mathrm{kg})^{8}$ & 46.7 & 0.7 & 27.3 & 0.9 \\
\hline Fat Free Mass $(\mathrm{kg})^{9}$ & 500.6 & 4.6 & 456.9 & 8.1 \\
\hline
\end{tabular}

1120

$1121{ }^{1}$ Reported measurements taken on d14 as horses exited the baseline sampling period

$1122{ }^{2}$ Reported measurements taken on $\mathrm{d} 98$ as horses exited the study

$1123{ }^{3}$ Assessment based on a 1-9 scale (Henneke et al., 1983)

$1124{ }^{4}$ Measured immediately behind slope of wither, caudal to elbow

$1125{ }^{5}$ Measured at two thirds distance from point of shoulder to point of hip

$1126{ }^{6}$ Measured by B-mode ultrasound, site determined by measuring half the distance from

1127 the point of the hip (tuber coxae) to the point of the buttock (ischiatic tuberosity); and

1128 half of the distance from this point to the spine, units expressed in $\mathrm{cm}$

$1129{ }^{7}$ Percent fat $=2.47+(5.47 \mathrm{x}$ rump fat thickness in $\mathrm{cm})$

$1130 \quad{ }^{8}$ Fat mass $=$ percent fat $\mathrm{x}$ body weight

$1131{ }^{9}$ Fat free mass = body weight - fat mass

1132 
1133 Figure 1 A-C: Changes in circulating oxidant status markers in relationship to change in

1134 body condition score and weight loss. Horses $(n=8)$ were enrolled in a baseline-

1135 sampling period lasting from $\mathrm{d} 0$ to $\mathrm{d} 14$, followed by a caloric restriction from $\mathrm{d} 15$ to $\mathrm{d} 98$,

1136 where from d 15 to $\mathrm{d} 43$ horses received a $30 \%$ digestible energy reduction, and from $\mathrm{d} 44$

1137 to $\mathrm{d} 98$ horses received a $30 \%$ restriction from maintenance digestible energy. On d 0, 14,

$113821,28,42,56,70$, and 98, blood samples were collected and analyzed for plasma nitrate

1139 concentration, erythrocyte glutathione concentration and erythrocyte glutathione

1140 peroxidase activity. The main effect of change in body condition score (BCS) and percent

1141 weight loss were analyzed for each variable, with data points representing values at

1142 corresponding points of BCS change or weight loss, respectively.

A

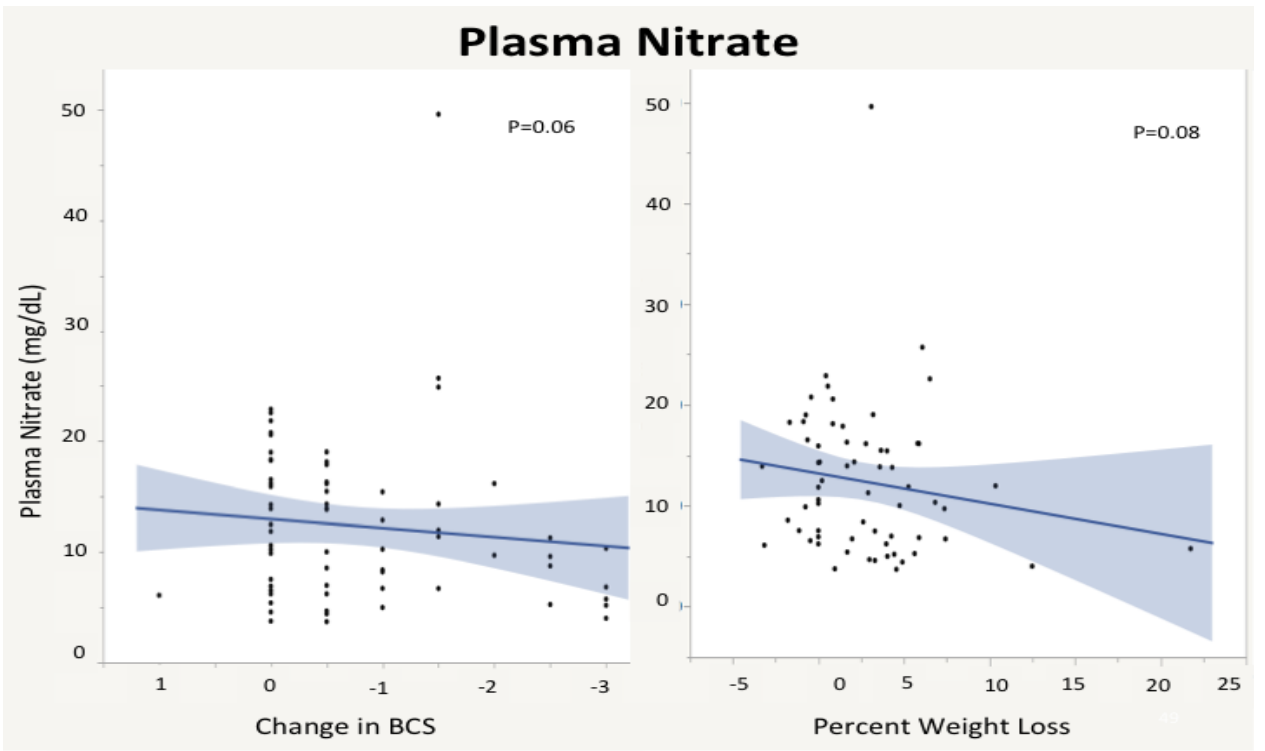

1143 


\section{Erythrocyte Glutathione}

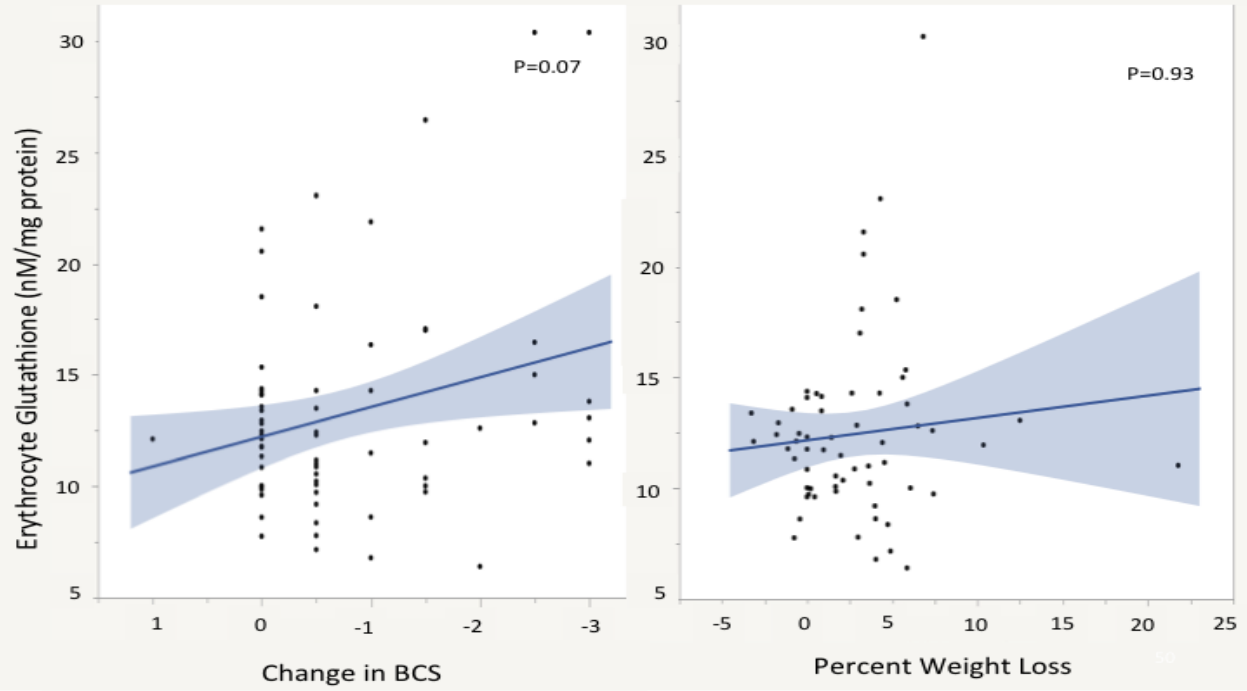

1145

1144

1146

C
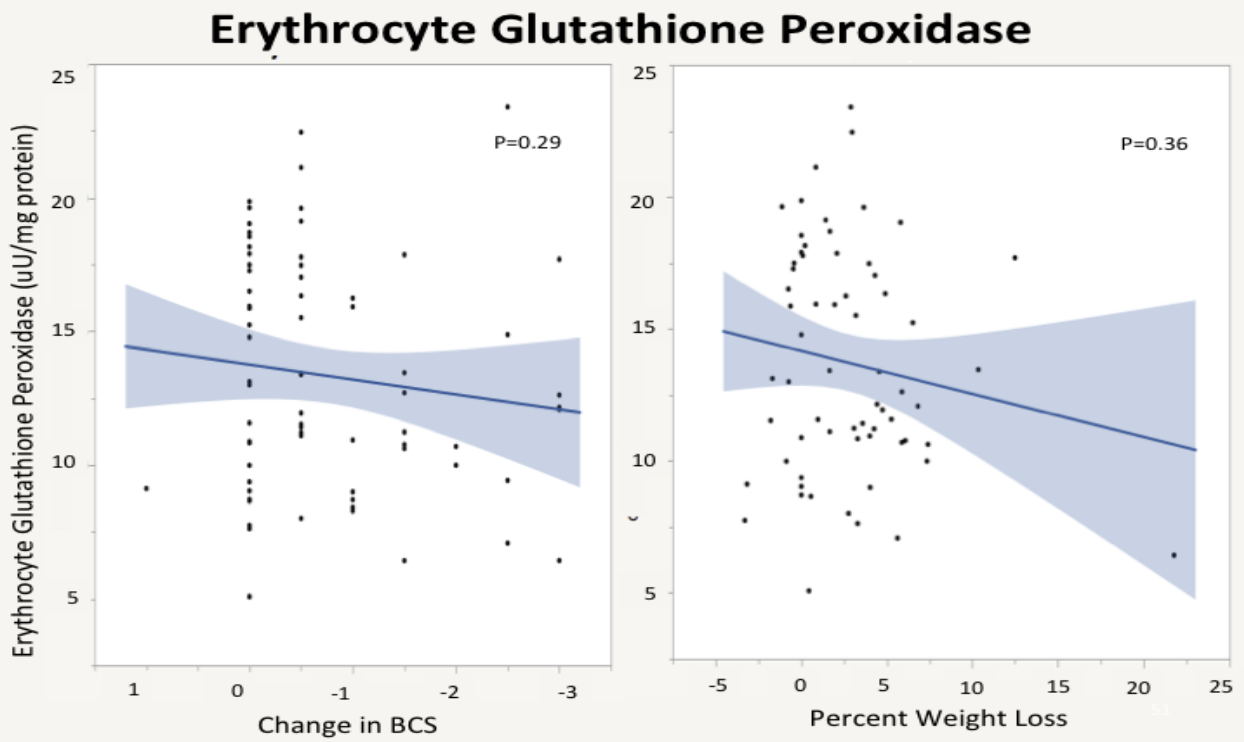

1148

1147

1149

1150

1151 
1152 Figure 2A-D: Mitochondrial Subpopulation Electron Transport Chain Enzyme Activities

1153 in Response to Change in Body Condition Score or Percent Weight Loss. Horses ( $\mathrm{n}=8)$

1154 were enrolled in a baseline-sampling period lasting from $\mathrm{d} 0$ to $\mathrm{d} 14$, followed by a caloric

1155 restriction from $\mathrm{d} 15$ to $\mathrm{d} 98$, where from $\mathrm{d} 15$ to $\mathrm{d} 43$ horses received a $30 \%$ digestible

1156 energy reduction, and from d44 to d 98 horses received a 30\% restriction from

1157 maintenance digestible energy. On d 0, 14, 28, 42, 70, and 98, middle gluteal muscle

1158 biopsies were taken and mitochondrial subpopulations isolated through differential

1159 centrifugation. Complex activities were measured spectrophotometrically depending on

1160 substrate production from each complex. The main effect of change in body condition

1161 score (BCS) and percent weight loss were analyzed for each variable, with data points

1162 representing values at corresponding points of BCS change or weight loss, respectively.

1163 Shading indicates confidence intervals.

A

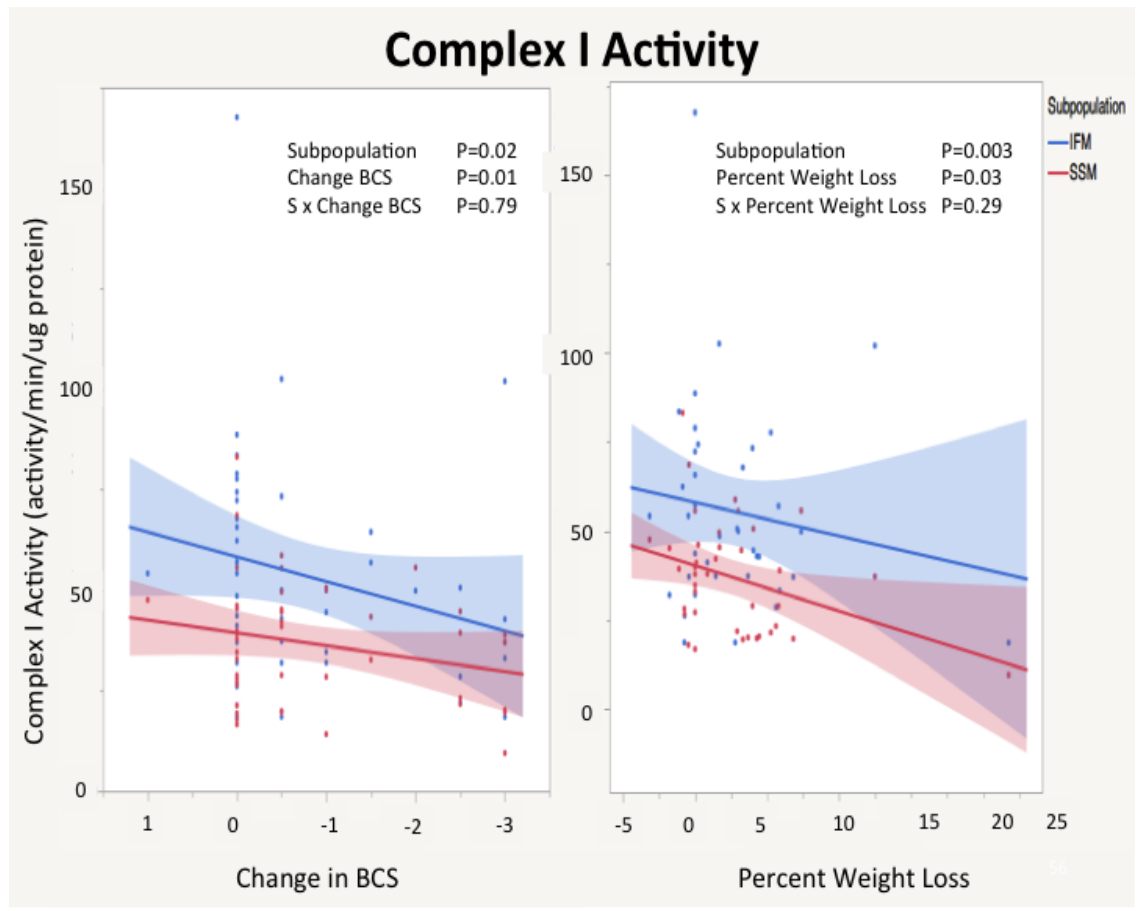

1164 
B

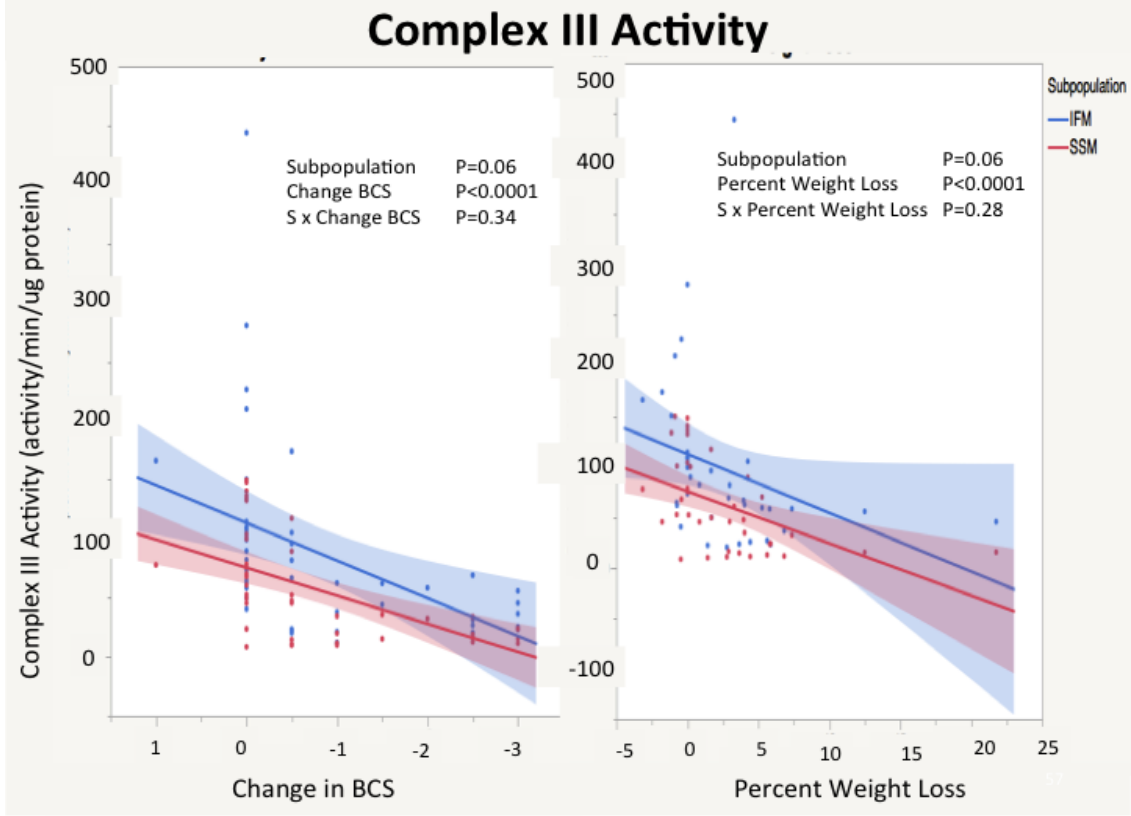

1165

C

\section{Complex IV Activitv}

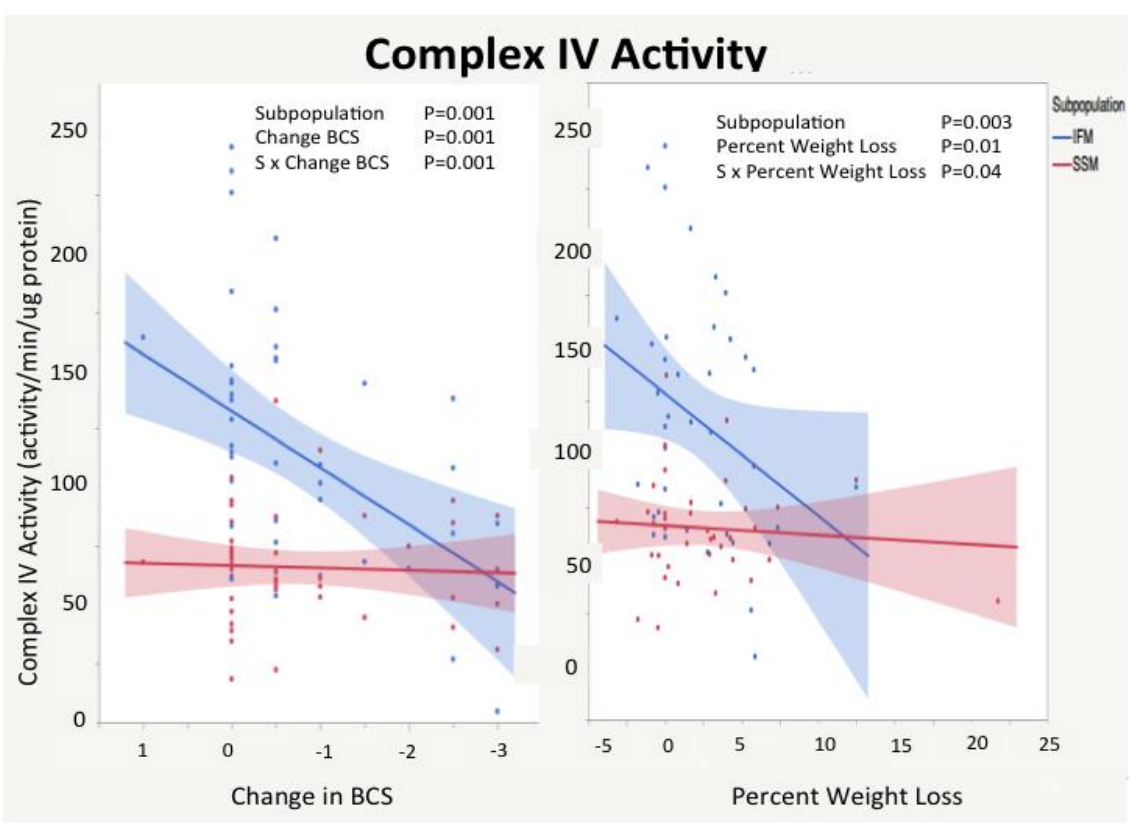


D

ATP Synthase Activity

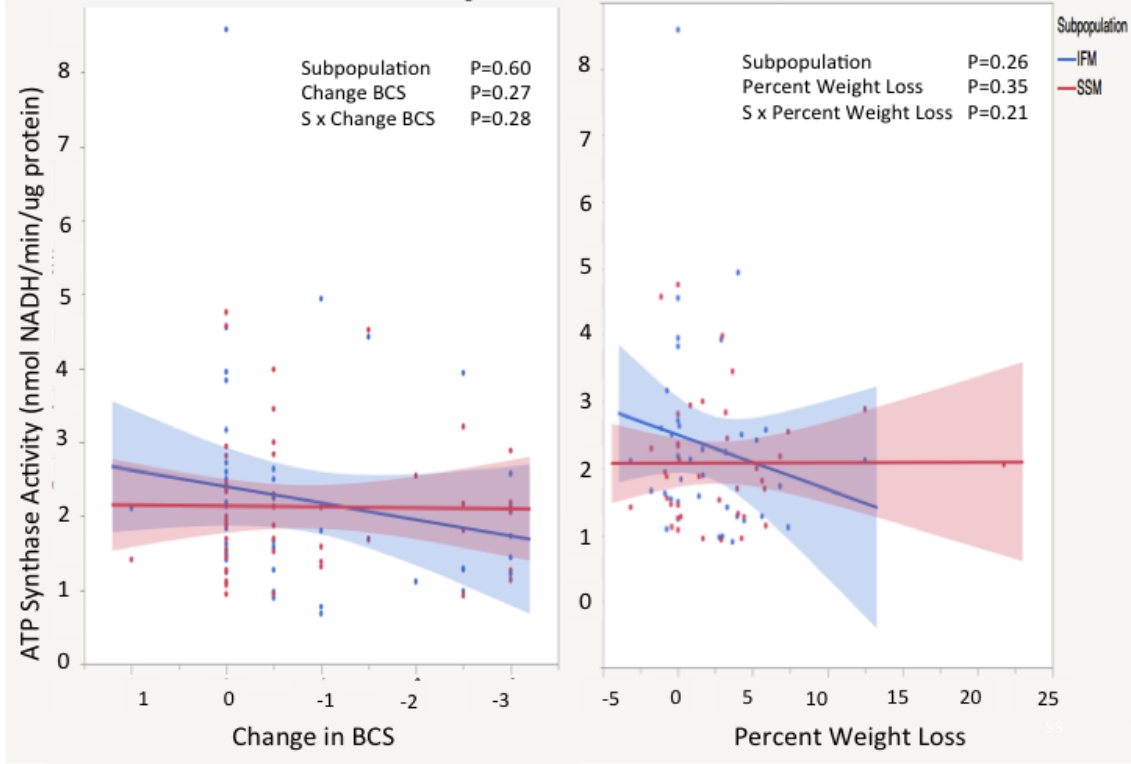

1169

1168

1170

1171 
1172 Figure 3: Mitochondrial subpopulation citrate synthase activity as an indicator of

1173 population size in response to change in body condition score or percent weight loss.

1174 Mitochondrial Subpopulation Electron Transport Chain Enzyme Activities in Response

1175 to Change in Body Condition Score or Percent Weight Loss. Horses $(n=8)$ were enrolled

1176 in a baseline-sampling period lasting from $\mathrm{d} 0$ to $\mathrm{d} 14$, followed by a caloric restriction

1177 from $\mathrm{d} 15$ to $\mathrm{d} 98$, where from $\mathrm{d} 15$ to $\mathrm{d} 43$ horses received a $30 \%$ digestible energy

1178 reduction, and from $\mathrm{d} 44$ to $\mathrm{d} 98$ horses received a $30 \%$ restriction from maintenance

1179 digestible energy. On d 0,14, 28, 42, 70, and 98, middle gluteal muscle biopsies were

1180 taken and mitochondrial subpopulations isolated through differential centrifugation.

1181 Citrate synthase activity was measured spectrophotometrically. The main effect of

1182 change in body condition score (BCS) and percent weight loss were analyzed for each

1183 variable, with data points representing values at corresponding points of BCS change or

1184 weight loss, respectively. Shading indicates confidence intervals.

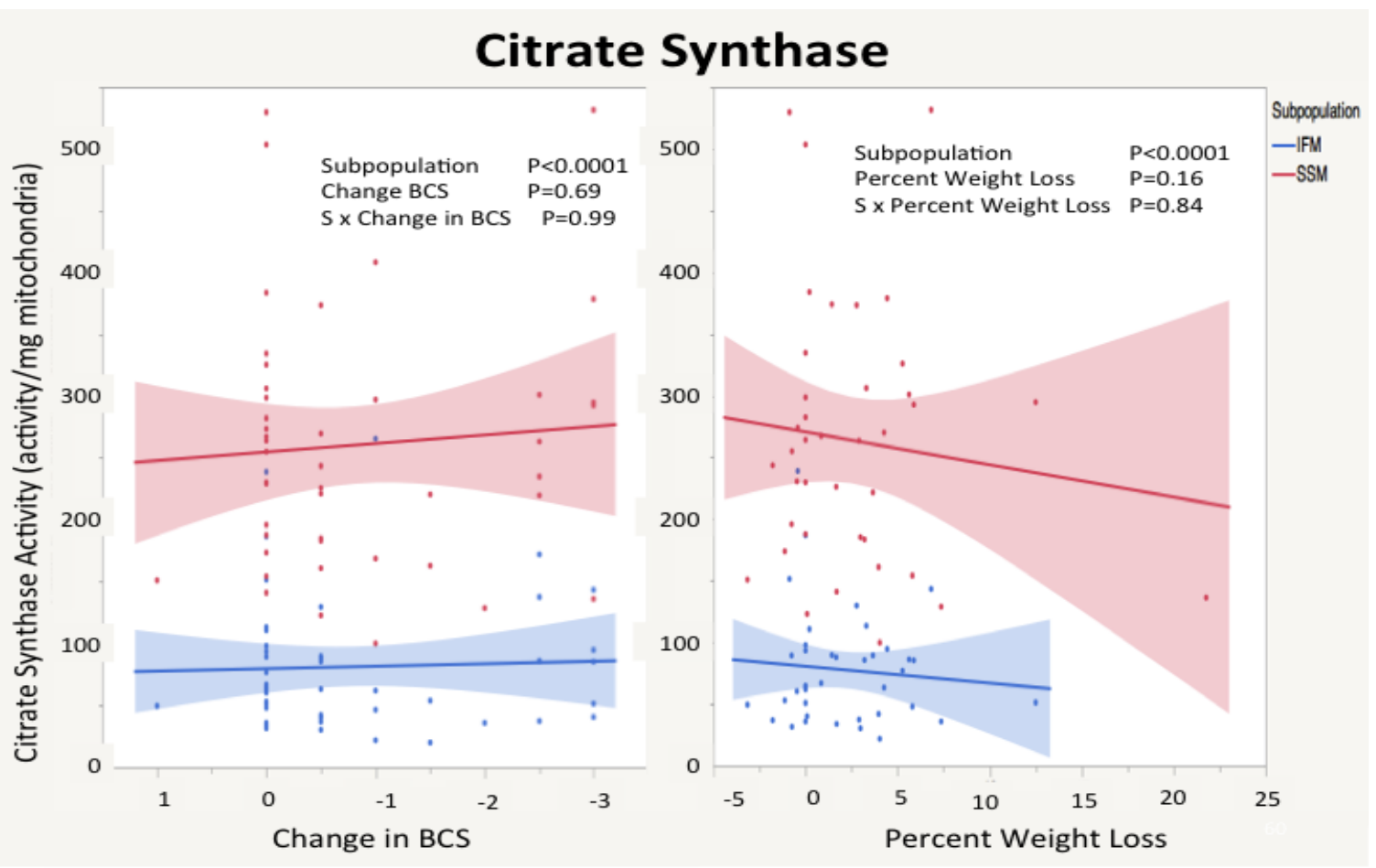


1186 Figure 4: Lipid peroxidation in mitochondrial subpopulations in response to change in

1187 body condition score or percent weight loss. Horses $(n=8)$ were enrolled in a baseline-

1188 sampling period lasting from $\mathrm{d} 0$ to $\mathrm{d} 14$, followed by a caloric restriction from $\mathrm{d} 15$ to $\mathrm{d} 98$,

1189 where from $\mathrm{d} 15$ to $\mathrm{d} 43$ horses received a 30\% digestible energy reduction, and from d44

1190 to d 98 horses received a 30\% restriction from maintenance digestible energy. On d 0, 14,

$119128,42,70$, and 98, middle gluteal muscle biopsies were taken and mitochondrial

1192 subpopulations isolated through differential centrifugation. Mitochondrial lipid

1193 peroxidation was measured spectrophotometrically, and was normalized to total lipid

1194 content determined via a sulfo-phosphovanillin assay. The main effect of change in body

1195 condition score (BCS) and percent weight loss were analyzed for each variable, with data

1196 points representing values at corresponding points of BCS change or weight loss,

1197 respectively. Shading indicates confidence intervals.

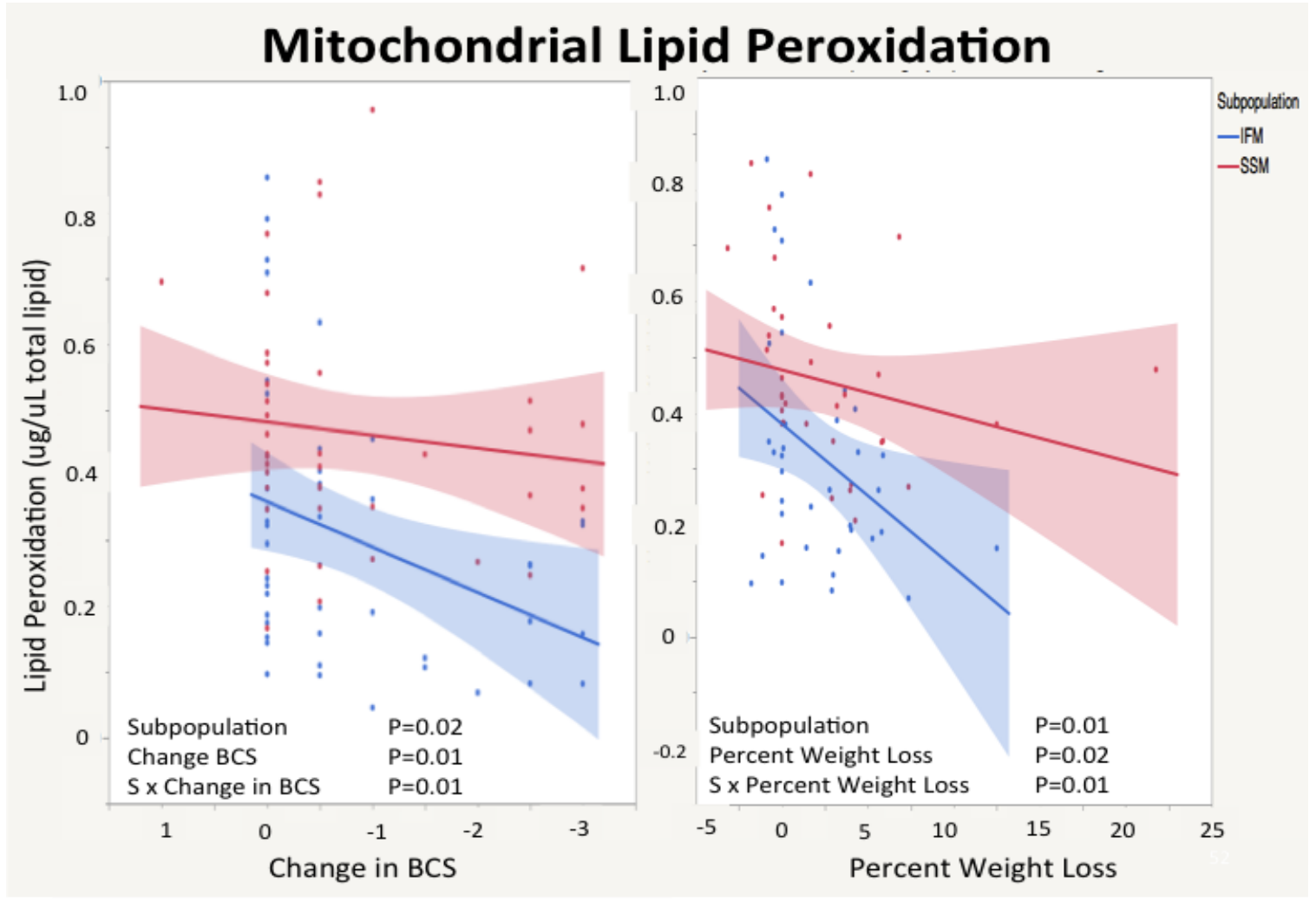


1199 Table 5: Correlation of Morphometric Measurements, Complex Activity and Circulating

1200 Oxidant Status Markers ${ }^{1}$

1201

\begin{tabular}{|c|c|c|c|c|c|c|c|c|c|c|c|c|}
\hline & \multicolumn{2}{|c|}{$\begin{array}{c}\text { Cresty Neck } \\
\text { Score }\end{array}$} & \multicolumn{2}{|c|}{ Nitric Oxide } & \multicolumn{2}{|c|}{ Glutathione } & \multicolumn{2}{|c|}{$\begin{array}{c}\text { Citrate Synthase } \\
\text { SSM }\end{array}$} & \multicolumn{2}{|c|}{$\begin{array}{l}\text { Lipid } \\
\text { Peroxidation } \\
\text { SSM }\end{array}$} & \multicolumn{2}{|c|}{$\begin{array}{c}\text { Lipid } \\
\text { Peroxidation } \\
\text { IFM }\end{array}$} \\
\hline & $\mathrm{R}$ & $P$ value & $\mathrm{R}$ & $P$ value & $\mathrm{R}$ & $P$ value & $\mathrm{R}$ & $P$ value & $\mathrm{R}$ & $P$ value & $\mathrm{R}$ & $P$ value \\
\hline Rump Fat Thickness & 0.63 & $<0.0001$ & 0.16 & 0.26 & -0.41 & 0.004 & -0.12 & 0.43 & 0.01 & 0.94 & 0.37 & 0.01 \\
\hline Percent Fat & 0.63 & $<0.0001$ & 0.15 & 0.31 & -0.42 & 0.003 & -0.13 & 0.41 & 0.03 & 0.88 & 0.38 & 0.01 \\
\hline Complex I - SSM & -0.09 & 0.53 & 0.33 & 0.02 & -0.25 & 0.09 & 0.12 & 0.42 & 0.17 & 0.29 & 0.37 & 0.01 \\
\hline Complex III - SSM & 0.46 & 0.002 & 0.06 & 0.69 & -0.27 & 0.06 & 0.07 & 0.65 & -0.04 & 0.8 & 0.40 & 0.01 \\
\hline Complex IV - SSM & -0.04 & 0.77 & 0.09 & 0.53 & -0.16 & 0.29 & -0.43 & 0.002 & 0.33 & 0.04 & 0.05 & 0.74 \\
\hline Complex IV - IFM & 0.43 & 0.003 & 0.06 & 0.72 & -0.05 & 0.75 & -0.16 & 0.3 & -0.33 & 0.04 & 0.06 & 0.69 \\
\hline Citrate Synthase - IFM & 0.04 & 0.77 & 0.24 & 0.09 & 0.19 & 0.19 & 0.61 & $<0.0001$ & -0.14 & 0.41 & 0.57 & $<0.0001$ \\
\hline Lipid Peroxidation - IFM & 0.35 & 0.02 & 0.46 & 0.002 & -0.22 & 0.14 & 0.51 & 0.0004 & 0.33 & 0.04 & & \\
\hline
\end{tabular}

$1204{ }^{1} \mathrm{P}$ values reflect significance and trends identified with bonferroni corrections, where

1205 significance determined at $\mathrm{p} \leq 0.0021$ and trends noted when $\mathrm{p} \leq 0.0052$. 
1218 As with any research project, limitations are inherently present and must be considered

1219 when interpreting results and looking forward to future research ideas. Specifically with

1220 regard to this project, limitations included sample size, time of sampling, species specific

1221 challenges, location of the project relative to analysis, and body condition of the subjects.

1222

1223 Inclusion criteria for this study resulted in a relatively small sample size relative to initial

1224 expected subject number. Upon analysis of results, specifically from both mitochondrial

1225 complex activities and minimal model output, large individual variation was observed.

1226 While consideration was taken to account for variability caused by gender, age, date and

1227 subpopulation where appropriate, additional comparisons may have been made had there

1228 been a larger total sampling population. For example, in the current study horses

1229 displayed a wide range of percent weight loss within observed BCS; had a larger sample

1230 size been possible, a natural break between "high" and "low" percentages of weight loss

1231 may have been revealed. The possibility remains that information regarding metabolic

1232 alterations with regard to weight loss is masked behind large variation. Increasing the

1233 sample size and allocating for natural breaks within the population could lend explanation

1234 to the variation observed in this study and alter overall interpretation of changes

1235 associated with obesity and subsequent weight loss.

1236

1237 Labor limitations and available stall space were also of concern during this study. All

1238 possible means to ensure horses were fed, managed and sampled in the same manner

1239 were taken, yet it was not fesable to collect fasting blood samples for analysis of 
1240 circulating metabolic indicators. Since samples were collected post-prandially, results

1241 were inconclusive. As the baseline FSIGT samples were collected after an overnight fast,

1242 we elected to evaluate these samples for circulating NEFA and TG levels (insulin and

1243 glucose concentrations measured for minimal model analysis). Unfortunately due to the

1244 timing of each FSIGT, this only provides for beginning and end-point information on

1245 these markers, leaving out samples from the weight loss period. Looking forward,

1246 management of horses so that fasting samples can be collected should be discussed for

1247 feasibility prior to the start of the research project. Additionally, inclusion of other

1248 markers that are not affected by fasting or fed states, including hemoglobin A1C or other

1249 glycosylated end products may also be of interest if fasting samples cannot be collected.

1250

1251 Unfortunately, while working with livestock species many analysis procedures do not

1252 transfer easily. For example, while hemoglobin A1C is considered a valid method for

1253 analyzing advancement of hyperglycemia in humans and rodents, initial attempts at

1254 transferring procedures to the horse have produced variable data. Additionally with

1255 regard to this study, measurement of specific oxidant status markers such as hydrogen

1256 peroxidase, manganese and copper/zinc superoxide dismutase have proven difficult to

1257 analyze with repeatable results. Analysis of genetic messages has also proven difficult, as

1258 the equine genome is not well annotated and many gene sequences are either unavailable

1259 or predicted. Thus, the measurements reported in this manuscript reflect assays

1260 previously validated in the horse. Further work is needed to accurately transition the

1261 aforementioned and other methodologies for analysis on equine samples. 
1263 In addition to the species-specific confines present in this study, travel limitations placed

1264 further restrictions on sample analysis procedures. As all samples were collected in

1265 Murfreesboro, TN and analysis was completed in Morgantown, WV samples were frozen

1266 at either -20 or $-80^{\circ} \mathrm{C}$ until transport and measurement was completed. Many

1267 measurements to analyze mitochondrial number, size, respiration and functionality must

1268 be completed on fresh, not frozen, isolations. Moreover, analysis of complex II activity

1269 requires fresh mitochondria and therefore was not feasibly possible during this study.

1270 Looking forward, consideration should be given to alternate methods of sampling or

1271 locations in order to fully analyze mitochondrial parameters.

1272

1273 As previously mentioned, horses were body condition scored prior to entry into the study.

1274 While we did account for overall obesity by using this method, hindsight suggests we

1275 should have also taken CNS into account. The horses on this project had CNS ranging

1276 between 2.5 to 3 during the baseline sampling period, which is considered a moderate

1277 score. Selecting horses with a CNS score of 3.5 or greater, it is possible we would have

1278 observed different results as horses would have been more obese. Further, as amount of

1279 neck crest fat can signify a transition into an unhealthy metabolic state (Carter et al.,

1280 2009), selecting horses with higher CNS could provide insight into the transition from

1281 "metabolically healthy obese" to "metabolically unhealthy". Degree of adiposity in the

1282 neck crest region should be considered when designing future studies, especially if

1283 metabolic dysfunction is to be evaluated. 
1285

1286

1287

1288 Obesity in the equine industry has continued to increase over time, without predisposition

1289 for breed, discipline or region. Viable information regarding the progression of metabolic

1290 changes associated with equine obesity and subsequent weight loss are critical to proper

1291 management and understanding of species-specific metabolic processes. Knowledge

1292 gained from this study has the capacity to influence not only nutritional interventions but

1293 also catalyze the industry's interpretation of equine performance and evolutionary

1294 advantages.

1295

1296 Evaluation of circulating biomarkers suggests horses were beginning to show signs of

1297 metabolic alterations as signified by the elevated nitrate and reduction in reduced

1298 glutathione concentrations. Combined with the lack of changes seen in most minimal

1299 model parameters, it is hypothesized that horses were metabolically healthy despite their

1300 obesity. Although cellular dysfunction is typically reflected in circulating markers, it is

1301 plausible that obesity-related metabolic changes were in the primary stages of

1302 development. Insulin sensitivity displayed improvements with weight loss, signifying

1303 some alteration in signaling or sensitivity at the tissue level, yet AIRg was not affected.

1304 Had a more advanced stage of obesity been allowed to develop, it is suspected that more

1305 advanced dysfunction would have developed. This "metabolically healthy obese"

1306 phenotype may be very delicate, easily transforming into a disease state, yet providing

1307 some insight as to compensatory mechanisms behind the commonly accepted

1308 dysfunctions associated with excessive adipose deposition. 
1310 Equine mitochondria is surprisingly unstudied, especially due to the competitive athletic

1311 nature of the equine industry. Based on the results of this study, it is evident that a

1312 species-related difference in coping mechanisms are present in the horse when directly

1313 compared to the human or rodent models. It is commonly regarded that horses provide

1314 an excellent model for human exercise performance and to a certain extent this theory

1315 remains true. While horses display a similar thermoregulatory system to that of a human,

1316 it is well known that horses possess numerous physiological adaptations contributing to

1317 their superior athletic ability. Without excessive speculation, it is feasible that the

1318 mitochondria also play a significant role in the performance of the equine athlete.

1319 Respiration levels of SSM and IFM from equine middle gluteal muscle compared to 1320 gastrocnemius and soleus mitochondrial subpopulation isolations C57/Black 6 displayed

1321 similar activity levels, yet substrate utilization time was much faster for both equine

1322 populations (Zambito et al., 2013).

1324 When combined with the data shown from this project, it is even more evident that horses

1325 display unique physiological mechanisms with respect to mitochondrial function. As

1326 horses are known for their ability to naturally blood dope via splenic contraction, the

1327 mitochondria may possess the ability to better handle large amounts of oxygen delivery

1328 without subsequent oxidative damage. This inherent protection may allow for equine

1329 mitochondria to possess more malleable characteristics to tolerate altering physiological

1330 environments. Return to a nearly equal level of complex activity between SSM and IFM

1331 displayed in this data could signify a more equal role between mitochondrial 
1332 subpopulations in a healthy state; a theory vastly differing from the suggested roles of

1333 SSM and IFM in humans and rodents alike (Kelley et al., 2002b; Menkishova et al.,

1334 2006; Ritov et al., 2005; Toledo et al., 2007). Unfortunately, due to lack of research on

1335 this specific topic, many of the proposed conclusions are based off negative comparisons

1336 with data in other species and not from completed works within the equine population.

1337 Mitochondrial research has an incredible depth of untapped potential due to the

1338 application of the knowledge base to athletic performance, cloning and reproduction and

1339 general animal health. Much more work is needed in this area to fill in the missing pieces

1340 as to the unique qualities of equine mitochondria.

1342 Overall, it is evident that differences within the obese horse population exist between

1343 those considered metabolically healthy and those displaying signs of severe metabolic

1344 impairment. Excessive fat deposition in specific regions, including the neck crest and

1345 abdomen, may require more attention when determining how to manage and care for

1346 obese horses. From a scientific standpoint, the central dogma surrounding overweight

1347 horses and the incidence of metabolic dysfunction may need to be re-evaluated with

1348 regard to physiological factors and onset of disease states.

1350 Looking forward, much work is needed with regard to mitochondrial function and obesity

1351 related metabolic changes in the horse. Classification of healthy equine mitochondria is

1352 necessary to understand not only the role of mitochondria on athletic performance but

1353 also propagation of disease states. Based on research in other species it remains plausible

1354 that understanding of many equine-related diseases can lie in evaluation of mitochondrial 
1355 function. Also, as regional fat deposition appears to play a role in obesity-related

1356 metabolic dysfunction research is needed to determine rates of deposition in different 1357 areas and their relationship to overall metabolism. Lastly, an in depth evaluation of the 1358 presence of a "metabolically healthy obese" phenotype is needed to classify how the 1359 industry manages obese horses. By understanding the physiology behind obesity related 1360 changes in glucose and lipid metabolism can drastically alter how we view the 1361 obese/overweight horse in both the performance and recreational setting.

1362 
1364

1365

1366

1367

1368

1369

1370

1371

1372

1373

1374

1375

1376

1377

1378

1379

1380

1381

1382

1383

1384

1385

1386

1387

1388

1389

1390

1391

1392

1393

1394

1395

1396

1397

1398

1399

1400

1401

1402

1403

1404

1405

Andrews, F. M., S. M. Reed, and G. C. Johnson. 1993. Muscle biopsy in the horse: its indications, techniques, and complications. . Veterinary Medicine 88: 357-365.

Attie, A. D., and P. E. Scherer. 2009. Adipocyte metabolism and obesity. Journal of Lipid Research April Supplement: S395-S399.

Bajpeyi, S. et al. 2011. Skeletal muscle mitochondrial capacity and insulin resistance in type 2 diabetes. Journal of Clinical Endocrinology and Metabolism 96: 11601168.

Barrientos, A. 2002. In vivo and in organello assessment of OXPHOS activities. Methods 26: 307-316.

Baseler, W. A. et al. 2011. Proteomic alterations of distinct mitochondrial subpopulaitons in the type I diabetic heart. American Journal of Physiology: Regulatory, Integrative and Comparative Physiology 300: 186-200.

Bender, M., and D. Smith. 1973. Classification of starch and fructosan-accumulating grasses as C3 or C4 species by caron isotope analysis. . Journal of British Grassland Society 28: 97-100.

Berggren, J. R., K. E. Boyle, W. H. Champan, and J. A. Houmard. 2008. Skeletal muscle lipid oxidation and obesity: influence of weight loss and exercise. American Journal of Physiology, Endocrinology and Metabolism 294: E726-E732.

Bizeau, M. E., W. T. Willis, and J. R. Hazel. 1998. Differential respinses to endurance training in subsarcolemmal and intermyofibrillar mitochondria. . Journal of Applied Physiology 85.

Bluher, M. 2010. The distinction of metabolically 'healthy' to 'unhealthy' obese individuals. . Current Opinion in Lipidology 21: 38-43.

Bluher, M. 2012. Are there still healthy obese patients? Current Opinion in Endocrinology: Diabetes and Obesity 19: 341-346.

Bonen, A. et al. 2004. Triacylglycerol accumulation in human obesity and type 2 diabetes is associated with increased rates of skeletal muscle fatty acid transport and increased sarcolemmal FAT/CD36. . Faseb Journal 18: 11441146.

Bonora, E. et al. 1991. U-shaped and J-shaped relationships between serum insulin and coronary heart disease in the general population. The Bruneck study. . Diabetes Care 21: 221-230.

Boston, R. C. et al. 2003. MINMOD Millennium: a computer program to calculate glucose effectiveness and insulin sensitivity from the frequently sampled intravenous glucose tolerance test. Diabetes Technol Ther 5: 1003-1015.

Bradford, M. M. 1976. A rapid and sensitive method for the quantification of microgram quantities of protein utilizing the principle of protein-dye binding. . Analytical Biochemistry 72: 248-254.

Bray, G. A. 2004. Medical consequences of obesity. Journal of Clinical Endocrinology Metabolism 89: 2583-2589.

Brownlee, M. 2001. Biochemistry and molecular cell biology of diabetic complications. Nature 414: 813-820. 
1406 1407 1408 1409 1410 1411 1412 1413 1414 1415 1416 1417 1418 1419 1420 1421 1422

1423 1424

1425

1426

1427

1428

1429

1430

1431

1432

1433

1434

1435

1436

1437

1438

1439

1440

1441

1442

1443

1444

1445

1446

1447

1448

1449

1450
Carter, R. A., R. J. Geor, W. Burton Staniar, T. A. Cubitt, and P. A. Harris. 2009. Apparent adiposity assessed by standardised scoring systems and morphometric measurements in horses and ponies. Vet J 179: 204-210.

Carter, R. A., J. McCutcheon, E. Valle, E. N. Meilahn, and R. J. Geor. 2010. Effects of exercise training on adiposity, insulin sensitivity, and plasma hormone and lipid concentrations in overweight or obese, insulin-resistant horses. American Journal of Veterinary Research 71: 314-321.

Cheng, Y. S., Y. Zheng, and J. S. VangerGheynst. 2011. Rapid quantitative analysis of lipids using a colorimetric method in a microplate format. Lipids 46: 95-103.

Cogswell, A. M., R. J. Stevens, and D. A. Hood. 1993. Properties of skeletal muscle mitochondria isolated from subsarcolemmal and intermyofibrillar regions. . American Journal of Physiology: Cell Physiology 253: C316-C322.

Consitt, L. A., J. A. Bell, and J. A. Houmard. 2009. Intramuscular lipid metabolism, insulin action and obesity. IUBMB Life 61: 47-55.

Cymbaluk, N. F., and G. I. Christison. 1990. Environmental effects on thermoregulation and nutition of horses. Veterinary Clinics of North America; Equine Practice 6: 355-372.

Dabkowski, E. R. et al. 2010. Mitochondrial dysfunction in the type 2 diabetic heart is associated with alterations in spatially distinct mitochondrial proteomes. American Journal of Physiology: Heart and Circulatory Physiology 299: H529H540.

Dabkowski, E. R. et al. 2009. Diabetic cardiomyopathy-associated dysfunction in spatially distinct mitochondrial subpopulations. . American Journal of Physiology: Heart and Circulation Physiology 296: H359-H369.

Dabkowski, E. R., C. L. Williamson, and J. M. Hollander. 2008a. Mitochondria-specific transgenic overexpression of phospholipid hydroperoxide glutathione peroxidase (GPX4) attenuates ischemia/reperfusion-associated cardiac dysfunction. . Free Radical Biology and Medicine 45: 855-865.

Dabkowski, E. R., C. L. Williamson, and J. M. Hollander. 2008b. Mitochondria-specific transgenic overexpression of phospholipid hydroperoxide glutathione peroxidase (GPX4) attenuates ischemia/reperfusion-associated cardiac dysfunction. Free Radical Biology and Medicine 45.

Denis, G. V., and M. S. Obin. 2013. 'Metabolically healthy obesity': Origins and implications. Molecular Aspects of Medicine 34: 59-70.

Feniouk, B. A., T. Suzuki, and Y. M. 2007. Regulatory interplay between proton motive force, ADP, phosphate and subunit epsilon in bacterial ATP synthase. Journal of Biological Chemistry 282: 764-772.

Fridovich, I. 1995. Superoxide radical and superoxide dismutases. Annual Review of Biochemisrty 64: 97-112.

Gentry, L. et al. 2002. The relationship between body condition, leptin, and reproductive and hormonal characteristics of mares during the seasonal anovulatory period. Journal of Animal Science 80: 2685-2703.

Geor, R. J., C. D. Thatcher, R. S. Pleasant, F. Elvinger, and L. Gay. 2007. Prevalence of hyperinsulinemia in mature horses: Relationship to adiposity. . Proceedings of the Annual Forum of the American College of Veterinary and Internal Medicine. 
1451 Goodyear, L. J., and B. B. Kahn. 1998. Exercise, glucoe transport, and insulin sensitivity. 1452 Annual Review of Medicine 49: 235-261.

Henneke, D. R., G. D. Potter, and J. L. Kreider. 1984. Body condition during pregnancy and lactation and reproductive efficiency of mares. . Theriogenology 21: 897909.

Henneke, D. R., G. D. Potter, J. L. Kreider, and B. F. Yeates. 1983. Relationship between condition score, physical measurements and body fat percentage in mares. Equine Vet J 15: 371-372.

Henry, M. D. 2003. Insulin resistance: From predisposing factor ro therapeutic target in type 2 diabetes. Clinical Therapeutics 25: B47-B63.

Hickey, M. S. et al. 1995. Skeletal muscle fiber composition is related to adiposity and in vitro glucose transport rate in humans. . American Journal of Physiology Endocrinology and Metabolism 268: E453-E457.

Hoffman, R. M., R. C. Boston, D. Stefanovski, D. S. Kronfeld, and P. A. Harris. 2003. Obesity and diet affect glucose dynamics and insulin sensitivity in Thoroughbred geldings. J Anim Sci 81: 2333-2342.

Holland, W. L. et al. 2007. Inhibition of ceramide synthesis ameliorates glucocorticoid-, saturated-fat-, and obesity-induced insulin resistance. . Cell Metabolism 5.

Holloway, G. P., A. Bonen, and L. L. Spiret. 2009. Regulation of skeletal muscle mitochondrial fatty acid metabolism in lean and obese individuals. The American Journal of Clinical Nutrition 89: 455S-462S.

Holloway, G. P., A. B. Thrush, and G. J. F. Heigenhauser. 2007. Skeletal muscle mitochondrial FAT/CD36 content and palmitate oxidation are not decreased in obese women. . The American Journal of Endocrine Metabolism 292: E1782E1789.

Hood, D. 2001. Plasticity in skeletal, cadriac, and smooth muscle: contractile activityinduced mitochondrial biogenesis in skeletal muscle. . Journal of Applied Physiology 90: 1137-1150.

Hoppeler, H. et al. 1987. Relating maximal oxygen consumption to skeletal muscle mitochondria in horses. Equine Exercise Physiology; ICEEP Proceedings: 278289.

Horowitz, J. F., and S. Klein. 2000. Whole body and abdominal lipolytic sensitivity to epinephrine is suppressed in upper body obese women. American Journal of Endocrinology and Metabolism 278: E1144-E1152.

Ippagunta, S., T. J. Hadenfeldt, J. L. Miner, and K. M. Hargrave-Barnes. 2011. Dietary conjugated linoleic acid induces lipolysis in adipose tissue of coconut oil-fed mice but not soy oil fed mice. Lipids 46: 821-830.

Kahn, B. B., and J. S. Flier. 2000. Obesity and insulin resistance. The Journal of Clinical Investigation 106: 473-481.

Kane, R. A., M. Fisher, D. Parrett, and L. M. Lawrence. 1987. Estimating fatness in horses. . Proceedings of the 10th Equine Nutrition and Physiology Symposium: 127-131.

Kayar, S. R., H. Hoppeler, S. L. Lindstedt, H. Claassen, and J. H. Jones. 1989. Total muscle mitochondrial volume in relation to aerobic capacity of horses and steers. Pflugers Archive 413: 407-413. 
1496

1497

1498

1499

1500

1501

1502

1503

1504

1505

1506

1507

1508

1509

1510

1511

1512

1513

1514

1515

1516

1517

1518

1519

1520

1521

1522

1523

1524

1525

1526

1527

1528

1529

1530

1531

1532

1533

1534

1535

1536

1537

1538

1539

Kearns, C. F., K. H. McKeever, and K. Malinowski. 2006a. Changes in adipopnectin, leptin, and fat mass after clenbuterol treatment in horses. Med Sci Sports Exerc 38: 262-267.

Kearns, C. F., K. H. McKeever, V. Roegner, S. M. Brady, and K. Malinowski. $2006 b$. Adiponectin and leptin are related to fat mass in horses. Vet J 172: 460-465.

Kelley, D. E., J. He, E. V. Menshikova, and V. B. Ritov. 2002a. Dysfunction of Mitochondria in Human Skeletal Muscle in Type 2 Diabetes 2: 2944-2950.

Kelley, D. E., J. He, E. V. Menshikova, and V. B. Ritov. 2002b. Dysfunction of mitochondria in human skeletal muscle in type 2 diabetes. Diabetes 51.

Kern, P. A., R. B. Simsolo, and M. Fournier. 1999. Effect of weight loss on muscle fiber type, fiber size capillarity, and succinate dehydrogenase activity in humans. Journal of Clinical Endocrinology Metabolism 84: 4185-4190.

Kim, J. Y., R. C. Hicker, R. L. Cortright, G. L. Dohm, and J. A. Houmard. 2000. Lipid oxidation is reduced in obese human skeletal muscle. . American Journal of Physiology and Endocrinology Metabolism. 279: E1039-E1044.

Kim, J. Y. et al. 2002. Evidence of malonyl-CoA-insensitive carnitine palmitoyltransferase I activity in red skeletal muscle. . American Journal of Physiology: Cell Physiology 251: C395-C402.

Kim, S. H., and G. M. Reaven. 2008. Insulin resisitance and hyperinsulinemia: you can't have one without the other. Diabetes Care 31: 1433-1438.

Kline, K. H., L. M. Lawrence, J. Novakofski, and P. J. Bechtel. 1987. Changes in muscle fiber type variation within the middle gluteal of young and mature horses as a function of sampling depth. Equine Exercise Physiology; ICEEP Proceedings: 271-277.

Kloting, N. et al. 2010. Insulin-sensitive obesity. . American Journal of Physiology; Endocrinology and Metabolism 299: E506-E515.

Kopelman, P. G. 2000. Obesity as a medical problem. Nature 404: 635-643.

Koves, T. R. et al. 2005. Subsarcolemmal and intermyofibrillar mitochondria play distinct roles in regulating skeletal muscle fatty acid metabolism. American Journal of Physiology: Cell Physiology 288: C1074-C1082.

Koves, T. R. et al. 2008. Mitochondrial overload and incomplete fatty acid oxidation contribute to skeletal muscle insulin resistance. . Cell Metabolism 7: 45-56.

Kreiger, D. A. 1980. Populations of rat skeletal muscle mitochondria after exercise and imobilization. Journal of Applied Physiology 48: 23-28.

Kronfeld, D. S. 2005. Insulin signaling laminitis and exercise. Journal of Equine Veterinary Science 25: 404-407.

Lamprecht, E. D., and C. A. Williams. 2012. Biomarkers of antioxidant status, inflammation, and cartilage metabolism are affected by acute intense exercise but not superoxide dismutase supplementation in horses. Oxid Med Cell Longev 2012: 920932.

Lawrence, L. M. et al. 1992. Observations on body weight and condition of horses in a 150-mile endurance ride. Journal of Equine Veterinary Science 12: 320-324.

Leibel, R. L., N. K. Edens, and S. K. Fried. 1989. Physiologic basis for the control of body fat in humans. Annual Reviews; Nutrition 9: 417-443. 
1540

1541

1542

1543

1544

1545

1546

1547

1548

1549

1550

1551

1552

1553

1554

1555

1556

1557

1558

1559

1560

1561

1562

1563

1564

1565

1566

1567

1568

1569

1570

1571

1572

1573

1574

1575

1576

1577

1578

1579

1580

1581

1582

1583

Lemasters, J. J. 2005. Selective mitochondrial autophagy, or mitophagy, as a targeted defense against oxidatve stress, mitochondrial dysfunction, and aging. Rejuvenation Research 8: 3-5.

Liburt, N. R. et al. 2012. The effect of age and exercise training on insulin sensitivity, fat and muscle tissue cytokine profiles and body composition of old and young Standardbred mares. Comparative Exercise Physiology 8: 173-187.

Lin, J. et al. 2002. Transcriptional co-activator PCG-1alpha drive the formation of slowtwitch muscle fibres. Nature 418: 797-801.

Lin, Y. et al. 2005. The hyperglycemia-induced inflammatory response in adipocytes: role of reactive oxygen species. Journal of Biological Chemistry 280: 4167-4626.

Manneschi, L., and A. Federico. 1995. Polargraphic analyses of subsarcolemmal and intermyofibrillar mitochondria from rat skeletal and cardiac muscle. . Journal of Neurology Science 128: 151-156.

Martinez, J. 2006. Mitochondrial oxidative stres and inflammation: a slalom to obesity and insulin resistance. Journal of Physiology and Biochemistry 62: 303-306.

McManus, C. J., and B. P. Fitzgerald. 2003. Effect of daily clenbuterol and expgenous melatonin treatment on body fat, serum leptin, and the expression of seasonal anestrous in the mare. Animal Reproduction Science 76: 217-230.

Menkishova, E. V. et al. 2006. Effects of weight loss and physical activity on skeletal muscle mitochondrial function in obesity. . American Journal of Physiology, Endocrinology and Metabolism 288: E818.

Montell, E., M. Turini, and M. Marotta. 2001. Diacylglycerol accumulation from saturated fatty acids desensitizes insulin stimulation of glucose uptake in muscle cells. . American Journal of Physiology and Endocrinology Metabolism. 280: E229-E237.

Morgunov, I., and P. A. Srere. 1998. Interaction between citrate synthase and malate dehydrogenase substrate channeling of oxaloacetate. . Journal of Biological Chemistry 273: 29540-29544.

Muoio, D. M., and C. B. Newgard. 2006. Obesity-related derangements in metabolic regulation. Annual Review of Biochemisrty 75: 367-401.

Nadler, S. T. et al. 2000. The expression of adipogenic genes is decreased in obesity and diabetes mellitus. Proceedings of the National Academies of the Sciences 97: 11371-11376.

Nielsen, B. D., C. I. O'Connor-Robison, H. S. Spooner, and J. Shelton. 2010. Glycemic and insulinemic responses are affected by age of horse and method of feed processing. Journal of Equine Veterinary Science 30: 249-258.

NRC. 2007. Nutrient Requirements of Horses, 6th Edition. The National Academies Press, Washington, D.C.

O'Connor, C. I., L. M. Lawrence, and S. H. Hayes. 2007. Dietary fish oil supplementation affects serum fatty acid concentrations in horses. J Anim Sci 85: 2183-2189.

$0^{\prime}$ Connor, C. I. et al. 2004. The effect of dietary fish oil supplementation on exercising horses. J Anim Sci 82: 2978-2984.

Ojima, K., and T. Isawa. 1968. The variation of carbohydrate in various species of grasses and legumes. . Canadian Journal of Botany 46. 
1584

1585

1586

1587

1588

1589

1590

1591

1592

1593

1594

1595

1596

1597

1598

1599

1600

1601

1602

1603

1604

1605

1606

1607

1608

1609

1610

1611

1612

1613

1614

1615

1616

1617

1618

1619

1620

1621

1622

1623

1624

1625

1626

1627

Palmer, J., B. Tandler, and C. Hoppel. 1977a. Biochemical properties of subsarcolemmal and interfibrillar mitochondria isolated from rat cadriac muscle. The Journal of Biological Chemistry 252: 8731-8739.

Palmer, J. W., B. Tandler, and C. L. Hoppel. 1985. Biochemical differences between subsarcolemmal and interfibrillar mitochondira from rat cardiac muscle: effects of procedural manipulations. Archives of Biochemistry and Biophysiology 236: 691-702.

Palmer, J. W., B. Tandler, and H. C. L. 1977b. Biochemical properties of subsarcolemmal and interfibrillarmitochondria isolated from rat cardiac muscle. . Journal of Biological Chemistry 252: 8731-8739.

Pedersen, M. et al. 2003. Circulating levels of TNF-a and IL-6 relation to truncal fat mass and muscle mass in healthy elderly individuals and in patients with type-2 diabetes. . Mechanics in Ageing and Development 124: 495-502.

Pleasant, R. S. et al. 2008. Prevalence of obesity in mature horses: an equine body condition study. Journal of Animal Physiology and Animal Nutrition. 92: 222.

Poole, D. 2004. Current concepts of oxygen transport during exercise. Equine Comparative Exercise Physiology 1: 5-22.

Pratt, S. E., R. J. Geor, and L. J. McCutcheon. 2005. Repeatability of 2 methods for assessment of insulin sensitivity and glucose dynamics in the horse. Journal of Veterinary Internal Medicine 19: 883-888.

Pullman, M. E., H. S. Penefsky, A. Datta, and E. Racker. 1960. Partial resolution of the enzymes catalyzing oxidative phosphorylation. I. Purification and properties of soluble dinitrophenol-stimulated adenosine triphosphate. Journal of Biological Chemistry 235: 3322-3329.

Quinn, R. W., A. O. Burk, J. K. Suagee, and T. G. Hartsock. 2006. Equine Obesity: Implications for health and strategies for control. Proceedings of the 4th MidAtlantic Nutrition Conference: 23-36.

Quiroz-Rothe, E., and J. L. Rivero. 2001. Co-coordinated expression of contractile and non-contractile features of control of equine muscle fibre types characterized by immunostaining of myosin heavy chains. . Histochemistry and Cell Biology 116: 299-312.

Reaven, G. M. (Editor), 1995. Insulin resistance and aging: modulation by obesity and physical activity. Perspective in Exercise Science and Sports Medicine, 8. Cooper Publishing Group, Traverse City, MI, 395-434 pp.

Ritov, V. B. et al. 2005. Deficiency of subsarcolemmal mitochondria in obesity and type 2 diabetes. Diabetes 84: 8-14.

Rosca, M. G. et al. 2009. Altered expression of the adenine nucleotide translocase isoforms and decreased ATP synthase activity in skeletal muscle mitochondria in heart failure. Journal of Molecular and Cellular Cardiology 46: 927-935.

Ryder, J. W., R. Bassel-Duby, E. N. Olson, and J. R. Zierath. 2003. Skeletal muscle reprogramming by activation of calcineurin improves insulin action on metabolic pathways. . Journal of Biological Chemistry 278: 44298-44304.

Scarpulla, R. C. 2008. Transcriptional paradigms in mammalian mitchondrial biogenesis and function. Physiological Review 88: 611-638. 
1628

1629

1630

1631

1632

1633

1634

1635

1636

1637

1638

1639

1640

1641

1642

1643

1644

1645

1646

1647

1648

1649

1650

1651

1652

1653

1654

1655

1656

1657

1658

1659

1660

1661

1662

1663

1664

1665

1666

1667

1668

1669

1670

1671

Schmitz-Peiffer, C. 2002. Protein kinase C and li[pid-induced insulin resisitance in skeletal muscle. . Annals of the New York Academy of the Sciences 967: 146157.

Sessions, D. R., S. E. Reedy, M. M. Vick, B. A. Murphy, and B. P. Fitzgerald. 2004. Development of a model for inducing transient insulin resistance in the mare: Preliminary implications regarding the estrous cycle. Journal of Animal Science 82: 2321-2328.

Shi, X. et al. 2008. Paridoxical effect of mitochondrial respiratroy chain impairment on insulin signaling and glucose transport in adipose cells. . Journal of Biological Chemistry 283: 30658-30667.

Simoneau, J. A., and D. E. Kelley. 1997. Altered glycolytic and oxidative capacities of skeletal muscle contribute to insulin resistance in NIDDM. Journal of Applied Physiology 83: 166-171.

Simoneau, J. A., J. H. Veerkamp, L. P. Turcotte, and D. E. Kelley. 1999. Markers of capacity to utilize fatty acids in human skeletal muscle: relation to insulin resistance and obesity and effects of weight loss. Faseb Journal 13: 2051-2060.

Sims, E. A. H. 2001. Are there persons who are obese but metabolically healthy? Metabolism 50: 1499-1504.

Smith, U., L. Stallones, W. H. Mueller, and B. H. Christensen. 1985. Regional differences in adipocyte metabolism and possible consequences in vivo. . International Journal of Obesity 9: 145-148.

Snow, D. H., and P. S. Guy. 1980. Muscle fiber type composition of a number of limb muscles in different types of horse. . Research in Veterinary Science 28: 137144.

Soukas, A. P., P. Cohen, N. D. Socci, and J. M. Friedman. 2000. Leptin-specific patterns of gene expresion in white adipose tissue. Genes and Development 14: 963-980.

Straub, S. G., and G. W. Sharp. 2002. Glucose-stimulated signaling pathways in biphasic insulin secretion. . Diabetes and Metabolism Research 18: 451-463.

Suagee, J. K. et al. 2008. Effects of diet and weight gain on body condition scoring in thoroughbred geldings. Journal of Equine Veterinary Science 28: 156-166.

Thornton, J., J. D. Pagan, and S. Persson. 1987. The oxygen cost of weight loading and inclined treadmill exercise in the horse. Equine Exercise Physiology 2 ICEEP Publication: 206.

Tinworth, K. D. et al. 2012. The effect of oral metformin on insulin sensitivity in insulinresistant ponies. The Veterinary Journal 191: 79-84.

Toledo, F. G. S., and B. H. Goodpaster. 2013. The role of weight loss and exercise in correcting skeletal muscle mitochondrial abnormalities in obesity, diabetes, and aging. . Molecular and Cellular Endocrinology: 2013.

Toledo, F. G. S. et al. 2007. Effects of Physical Activity and Weight Loss on Skeletal Msucle Mitochondria and Relationship With Glucose Control in Type 2 Diabetes. Diabetes 56: 2142-2147.

Treiber, K. et al. 2006. Evaluation of genetic and metabolic predispositions and nutritional risk facotrs for pasture-associated laminits in ponies. Journal of the American Veterinary Medical Association 228: 357-365. 
1672

1673

1674

1675

1676

1677

1678

1679

1680

1681

1682

1683

1684

1685

1686

1687

1688

1689

1690

1691

1692

1693

1694

1695

1696

1697

1698

1699

1700

1701

1702

1703

1704

1705

1706

1707

1708
Trounce, I. A., K. Y., A. S. Jun, and D. C. Wallace. 1996. Assessment of nitochonrial oxidative phosphrylation in patient muscle biopsies, lymphoblasts and transmitochondrial cell lines. . Methods 264: 484-509.

Valberg, S., and A. Firshman. 2009. Insulin resistance: What is it and how do we measure it? . Kentucky Equine Research, Inc.: 335-366.

Valerio, A. et al. 2006. TNF-a downregulates eNOS expression and mitochondrial biogenesis in fat and muscle of obese rodents. Journal of Clinical Investigation 116: 2791-2798.

Votion, D. M., E. Gnaiger, H. Lemieux, A. Mouithys-Mickalad, and D. Serteyn. 2012. Physical fitness and mitochondrial respiratory capacity in horse skeletal muscle. PLOS One 7: 1-12.

Watson, R. T., and J. E. Pessin. 2001. Intracellular organization of insulin signaling and GLUT4 translocation. Recent Progress in Hormone Research 56: 175-194.

Webb, S. P., G. D. Potter, J. W. Evans, and G. W. Webb. 1989. Influence of body fat content on digestible energy requirements of exercising horses in temperate and hot environments. . Proceedings of the 11th Equine Nutrition and Physiology Symposium: 279-284.

Westervelt, R. G., J. R. Stouffer, H. F. Hintz, and H. F. Schryver. 1976. Estimating fatness in horses and ponies. Journal of Animal Science 43: 781-785.

Wildman, R. P. et al. 2008. The obese without cardiometabolic risk fasctor clustering and the normal weight with cardiometabolic risk factor clustering: prevalence and correlates of 2 phenotypes among US population (NHANES 1999-2004). Archives of Internal Medicine 168: 1617-1624.

Williamson, C. L. et al. 2009. Enhanced apoptotic propensity in diabetic cardiac mitochondria: influence of subcellular spatial location. American Journal of Heart Circulatory Physiology 298: H633-H642.

Yki-Jarven, H. 1992. Glucose toxicity. Endocrinology review. 3: 415-431.

$Y$ u, C. et al. 2002. Mechanism by which fatty acids inhibit insulin activation of insulin receptor substrate-1 (IRS-1)-associated phosphotidylinositol 3-kinase activity in muscle. Journal of Biological Chemistry 277: 50230-50236.

Zambito, J. L., C. E. Nichols, H. S. Spooner, K. M. Barnes, and J. M. Hollander. 2013. Novel evaluation of equine and murine skeletal muscle mitochondrial function: electron transport chain activity and oxygen consumption. Journal of Equine Veterinary Science 33: 326-327. 


\title{
EDUCATION
}

\section{Jennie L. Zambito, M.S., PAS}

\author{
West Virginia University, Morgantown, WV \\ Davis College of Agriculture, Natural Resources, and Design \\ Doctor of Philosophy, Animal and Nutritional Sciences \\ Equine Exercise Physiology and Nutrition \\ Ph.D. Candidate; Expected Completion December 2013 \\ West Virginia University, Morgantown, WV \\ Davis College of Agriculture, Natural Resources, and Design \\ Masters of Science, Animal and Nutritional Sciences \\ Equine Exercise Physiology and Nutrition \\ Graduated August 2011
}

Rutgers, The State University of New Jersey, New Brunswick, NJ

School of Environmental and Biological Sciences

B.S., Animal Science, Pre-Veterinary Medicine/Research, Equine Science Graduated May 2009

\section{TEACHING \\ EXPERIENCE}

West Virginia Junior College, Nursing Program; Morgantown, WV -February 2012 - March 2012

BIO 181 Introduction to Microbiology: Instructor

-September 2011- February 2012

ANP 181, 182 and 183 Anatomy and Physiology 1, 2, and 3: Instructor

West Virginia University, Davis College; Morgantown, WV

-Fall 2013, Summer 2013, Fall 2012

AGBI 410 Introduction to Biochemistry: Teaching Assistant

-Spring 2012, Spring 2011, Spring 2010

ANPR 344 Light Horse Science: Teaching Assistant

-Fall 2011, Fall 2010, Fall 2009

A\&VS 281 Equine Management and Training: Teaching Assistant A\&VS 493 Equine Exercise Physiology: Teaching Assistant 
1754

1755

1756

1757

1758

1759

1760

1761

1762

1763

1764

1765

1766

1767

1768

1769

1770

1771

1772

1773

1774

1775

1776

1777

1778

1779

1780

1781

1782

1783

1784

1785

1786

1787

1788

1789

1790

1791

1792

1793

1794

1795

1796

1797

1798

1799

-Spring 2011

A\&VS 293V Veterinary Anatomy Lab: Teaching Assistant

-Fall 2010

A\&VS 293W Equestrian Technologies: Instructor

A\&VS 293C Equine Safety and Handling: Instructor

-Summer 2010

A\&VS 293 Independent Study: Teaching Assistant

-Spring 2010

A\&VS 293W Equestrian Technologies: Teaching Assistant

\section{RESEARCH PUBLICATIONS AND PRESENTATIONS}

Changes in Oxidant Status in Response to Weight Loss in Mature Light-Type Horses

EH Hoblitzell, KM Barnes, JL Zambito, HS Spooner

Submitted to 2014 Midwest ASDS ASAS Meeting

Effects of Weight Loss on Lipolysis and Skeletal Muscle Genetic Markers of Beta-Oxidation

M Bush, KM Barnes, JL Zambito, HS Spooner

Submitted to the Experimental Biology 2014 Meeting

Weight Loss Influence on Glucose Tolerance and Metabolic Markers in the Mature Horse

JL Zambito, HS Spooner, RM Hoffman, KM Barnes

Submitted to the Experimental Biology 2014 Meeting

Influence of Weight Loss on Mitochondrial Function in the Mature Horse

JL Zambito, CE Nichols, KM Barnes, HS Spooner, JM Hollander

Submitted to the International Conference on Equine Exercise Physiology 2015

Meeting

Effects of Resveratrol Supplementation on Glycemic Response and Oxidant Status in Exercising Aged Quarter Horse Geldings

JL Zambito, HS Spooner, RM Hoffman

Presented at the 2012 Joint Annual Meeting, Phoenix, AZ

Effects of Oral Resveratrol Supplementation on Insulin Sensitivity and Reproductive Cyclicity in Obese Mares on Pasture KS Kohlhass, JL Zambito, HS Spooner, RC Boston, ML Rhodes, JW Knight, RK Splan 
Journal of Equine Veterinary Science, 33:370-371, 2013

Comparison of Krill Oil and Fish Oil Supplementation on Serum and Tissue

LE Bowen, HS Spooner, JL Zambito, KM Barnes

Journal of Equine Veterinary Science, 33: 342, 2013

Effects of Food Extracts on Plasma Electrolyte Concentration and Strong Ion

JL Zambito, KH McKeever, JM Streltsova, NR Liburt

Journal of Equine Veterinary Science, 29:479-480, 2009.

1812

1813

The Effect of Age and Exercise Training on Insulin Sensitivity, Fat and Muscle 


\begin{tabular}{|c|c|c|c|c|c|c|c|c|}
\hline Horse & Day & $\begin{array}{l}\text { Plasma } \\
\text { Nitrate } \\
(\mathrm{mg} / \mathrm{dL})\end{array}$ & $\begin{array}{l}\text { Erythrocyte } \\
\text { Glutathione } \\
\text { (nMol/mg } \\
\text { protein) }\end{array}$ & $\begin{array}{c}\text { Erythrocyte } \\
\text { Glutathione } \\
\text { Peroxidase } \\
\text { (uU/mg } \\
\text { protein) }\end{array}$ & $\begin{array}{c}\text { Serum } \\
\text { NEFA } \\
(\mathrm{mg} / \\
\mathrm{mL})\end{array}$ & $\begin{array}{l}\text { Plasma } \\
\text { TG } \\
(\mathrm{mg} / \\
\mathrm{mL})\end{array}$ & $\begin{array}{l}\text { Glucose } \\
(\mathrm{mg} / \mathrm{dL})\end{array}$ & $\begin{array}{l}\text { Insulin } \\
(\mathrm{mU} / \mathrm{L})\end{array}$ \\
\hline Boots & 0 & 4.57 & 21.56 & 0.0076 & 0.483 & 12.22 & 108.69 & 2.80 \\
\hline Boots & 14 & 7.52 & 14.09 & 0.0090 & 0.188 & 21.98 & 127.07 & 4.82 \\
\hline Boots & 21 & 7.48 & 20.56 & 0.0108 & 0.192 & 34.22 & 126.09 & 4.45 \\
\hline Boots & 28 & 11.87 & 18.51 & 0.0116 & 0.306 & 24.72 & 108.87 & 3.69 \\
\hline Boots & 35 & 22.57 & 12.81 & 0.0152 & 0.124 & 38.44 & 133.17 & 5.27 \\
\hline Boots & 42 & 16.17 & 15.34 & 0.0190 & 0.354 & 22.98 & 111.04 & 7.18 \\
\hline Boots & 56 & 11.97 & 11.95 & 0.0135 & 0.114 & 17.46 & 127.33 & 2.38 \\
\hline Boots & 70 & 24.89 & 26.44 & 0.0064 & 0.276 & 34.68 & 95.91 & 2.39 \\
\hline Boots & 98 & 4.00 & 13.06 & 0.0177 & 0.240 & 10.32 & 114.81 & 2.68 \\
\hline Daphne & 0 & 20.76 & 8.61 & 0.0175 & 0.075 & 18.48 & 113.65 & 12.70 \\
\hline Daphne & 14 & 14.27 & 10.02 & 0.0109 & 0.074 & 46.32 & 91.63 & 6.16 \\
\hline Daphne & 21 & 16.52 & 12.12 & 0.0159 & 0.297 & 20.84 & 104.55 & 4.62 \\
\hline Daphne & 28 & 12.48 & 9.98 & 0.0182 & 0.157 & 18.1 & 112.52 & 5.04 \\
\hline Daphne & 35 & 10.01 & 8.36 & 0.0119 & 0.451 & 149.32 & 122.64 & 4.47 \\
\hline Daphne & 42 & 5.40 & 9.86 & 0.0187 & 0.131 & 18.4 & 107.54 & 6.55 \\
\hline Daphne & 56 & 15.43 & 8.62 & 0.0109 & 0.114 & 31.46 & 143.55 & 3.31 \\
\hline Daphne & 70 & 10.25 & 14.26 & 0.0083 & 0.094 & 21.7 & 134.84 & 7.27 \\
\hline Daphne & 98 & 5.18 & 12.06 & 0.0121 & 0.239 & 35.94 & 133.27 & 9.22 \\
\hline Getda & 0 & 18.33 & 13.56 & 0.0100 & 0.575 & 18.46 & 96.39 & 3.88 \\
\hline Getda & 14 & 15.92 & 12.31 & 0.0087 & 0.080 & 22.42 & 118.05 & 17.82 \\
\hline Getda & 21 & 21.84 & 14.27 & 0.0087 & 0.258 & 25.7 & 93.51 & 2.47 \\
\hline Getda & 28 & 16.29 & 10.07 & 0.0134 & 0.316 & 26.34 & 151.36 & 1.84 \\
\hline Getda & 35 & 8.39 & 14.29 & 0.0162 & 0.432 & 38.82 & 99.73 & 3.16 \\
\hline Getda & 42 & 16.14 & 10.87 & 0.0080 & 0.413 & 22.92 & 121.79 & 3.15 \\
\hline Getda & 56 & 49.56 & 17.00 & 0.0112 & 0.113 & 29.84 & 125.06 & 2.57 \\
\hline Getda & 70 & 8.75 & 30.38 & 0.0094 & 0.248 & 31.22 & 194.11 & 2.23 \\
\hline Getda & 98 & 10.33 & 30.38 & 0.0121 & 0.057 & 13.22 & 101.26 & 8.76 \\
\hline JR & 0 & 6.08 & 12.11 & 0.0091 & 0.073 & 27.82 & 110.82 & 1.99 \\
\hline JR & 14 & 6.21 & 11.76 & 0.0179 & 0.172 & 45.86 & 112.26 & 1.97 \\
\hline JR & 21 & 3.74 & 11.73 & 0.0116 & 0.157 & 33.6 & 97.53 & 3.71 \\
\hline JR & 28 & 4.66 & 7.79 & 0.0224 & 0.157 & 39.06 & 79.35 & 2.02 \\
\hline JR & 35 & 4.41 & 7.16 & 0.0163 & 0.182 & 29.06 & 43.48 & 2.02 \\
\hline JR & 42 & 4.98 & 6.79 & 0.0090 & 0.265 & 26.68 & 108.36 & 2.27 \\
\hline JR & 56 & 16.15 & 6.40 & 0.0107 & 0.073 & 17.34 & 99.85 & 2.31 \\
\hline JR & 70 & 9.70 & 12.60 & 0.0100 & 0.280 & 20.7 & 112.26 & 2.04 \\
\hline
\end{tabular}




\begin{tabular}{|c|c|c|c|c|c|c|c|c|}
\hline Horse & Day & $\begin{array}{l}\text { Plasma } \\
\text { Nitrate } \\
(\mathrm{mg} / \mathrm{dL})\end{array}$ & $\begin{array}{c}\text { Erythrocyte } \\
\text { Glutathione } \\
\text { (nMol/mg } \\
\text { protein) }\end{array}$ & $\begin{array}{c}\text { Erythrocyte } \\
\text { Glutathione } \\
\text { Peroxidase } \\
\text { (uU/mg } \\
\text { protein) }\end{array}$ & $\begin{array}{c}\text { Serum } \\
\text { NEFA } \\
(\mathrm{mg} / \\
\mathrm{mL})\end{array}$ & $\begin{array}{l}\text { Plasma } \\
\text { TG } \\
(\mathrm{mg} / \\
\mathrm{mL})\end{array}$ & $\begin{array}{l}\text { Glucose } \\
(\mathrm{mg} / \mathrm{dL})\end{array}$ & $\begin{array}{l}\text { Insulin } \\
(\mathrm{mU} / \mathrm{L})\end{array}$ \\
\hline Pistol & 0 & 8.56 & 12.42 & 0.0115 & 0.176 & 8.04 & 97.36 & 4.09 \\
\hline Pistol & 14 & 10.59 & 10.85 & 0.0148 & 0.064 & 15.22 & 161.46 & 7.09 \\
\hline Pistol & 21 & 13.91 & 13.39 & 0.0077 & 0.144 & 20.22 & 108.73 & 3.52 \\
\hline Pistol & 28 & 7.53 & 11.78 & 0.0196 & 0.072 & 16.72 & 103.97 & 4.46 \\
\hline Pistol & 35 & 18.12 & 13.49 & 0.0211 & 0.179 & 23.18 & 100.17 & 2.77 \\
\hline Pistol & 42 & 6.53 & 12.47 & 0.0173 & 0.170 & 28.82 & 108.79 & 5.20 \\
\hline Pistol & 56 & 6.71 & 11.48 & 0.0159 & 0.068 & 19.68 & 128.33 & 2.98 \\
\hline Pistol & 70 & 8.21 & 21.88 & 0.0087 & 0.253 & 30.82 & 126.45 & 6.11 \\
\hline Pistol & 98 & 11.28 & 12.84 & 0.0234 & 0.158 & 15.96 & 145.46 & 8.29 \\
\hline Romeo & 0 & 20.57 & 14.14 & 0.0159 & 0.184 & 26.06 & 113.14 & 6.99 \\
\hline Romeo & 14 & 11.83 & 14.37 & 0.0199 & 0.073 & 23.72 & 152.51 & 11.42 \\
\hline Romeo & 21 & 13.79 & 23.06 & 0.0170 & 0.291 & 31.96 & 116.67 & 4.12 \\
\hline Romeo & 28 & 19.02 & 18.08 & 0.0155 & 0.318 & 30.96 & 74.13 & 2.23 \\
\hline Romeo & 35 & 13.84 & 11.00 & 0.0114 & 0.389 & 35.56 & 114.11 & 2.74 \\
\hline Romeo & 42 & 15.48 & 10.22 & 0.0196 & 0.198 & 27.2 & 117.78 & 3.35 \\
\hline Romeo & 56 & 25.70 & 10.01 & 0.0108 & 0.223 & 33.58 & 148.08 & 2.61 \\
\hline Romeo & 70 & 9.59 & 16.46 & 0.0149 & 0.154 & 38.04 & 136.13 & 1.62 \\
\hline Romeo & 98 & 6.83 & 13.80 & 0.0126 & 0.116 & 22.84 & 151.83 & 3.22 \\
\hline Susie & 0 & 9.88 & 7.76 & 0.0165 & 0.127 & 11.82 & 99.98 & 7.08 \\
\hline Susie & 14 & 10.23 & 11.74 & 0.0185 & 0.044 & 17.58 & 115.04 & 8.46 \\
\hline Susie & 21 & 18.26 & 12.95 & 0.0131 & 0.234 & 19.96 & 38.89 & 5.99 \\
\hline Susie & 28 & 14.32 & 9.72 & 0.0178 & 0.163 & 22.96 & 106.75 & 4.06 \\
\hline Susie & 35 & 13.94 & 10.55 & 0.0111 & 0.119 & 13.98 & 91.01 & 5.00 \\
\hline Susie & 42 & 17.87 & 12.29 & 0.0191 & 0.206 & 10.3 & 107.77 & 5.31 \\
\hline Susie & 56 & 14.33 & 10.36 & 0.0179 & 0.061 & 22.44 & 102.77 & 6.31 \\
\hline Susie & 70 & 12.87 & 16.35 & 0.0084 & . & 25.84 & 129.14 & 5.86 \\
\hline Susie & 98 & 5.25 & 15.00 & 0.0071 & 0.125 & 30.7 & 111.20 & 5.53 \\
\hline Sweetie & 0 & 18.99 & 11.33 & 0.0130 & 0.149 & 29.18 & 104.77 & 9.02 \\
\hline Sweetie & 14 & 6.91 & 9.61 & 0.0094 & 0.131 & 29.98 & 107.61 & 19.05 \\
\hline Sweetie & 21 & 22.88 & 9.61 & 0.0051 & 0.255 & 30.82 & 139.30 & 7.06 \\
\hline Sweetie & 28 & 6.98 & 14.29 & 0.0112 & 0.273 & 42.96 & 125.60 & 8.49 \\
\hline Sweetie & 35 & 3.69 & 11.16 & 0.0134 & 0.106 & 22.72 & 96.55 & 7.51 \\
\hline Sweetie & 42 & 6.21 & 9.20 & 0.0175 & 0.060 & 22.22 & 118.33 & 5.82 \\
\hline Sweetie & 56 & 6.70 & 9.74 & 0.0106 & 0.107 & 26.1 & 95.12 & 6.53 \\
\hline Sweetie & 70 & 11.40 & 17.07 & 0.0127 & 0.225 & 23.2 & 147.66 & 2.45 \\
\hline Sweetie & 98 & 5.74 & 11.03 & 0.0064 & 0.228 & 22.72 & 131.99 & 3.37 \\
\hline
\end{tabular}


Appendix B

\begin{tabular}{cccccccc}
\hline \hline Horse & $\begin{array}{c}\text { Horse } \\
\text { Number }\end{array}$ & $\begin{array}{c}\text { Age } \\
(\mathrm{yr})\end{array}$ & Sex & Breed & $\begin{array}{c}\text { Wither } \\
\text { Height }\end{array}$ & $\begin{array}{c}\text { Body } \\
\text { Length }\end{array}$ & $\begin{array}{c}\text { Neck } \\
\text { Length }\end{array}$ \\
\hline Boots & 1 & 10 & $\mathrm{M}$ & Quarter Horse & 142.2 & 157.5 & 71.1 \\
Daphne & 2 & 6 & $\mathrm{M}$ & Tennessee Walker & 148.0 & 137.2 & 71.1 \\
Getda & 3 & 13 & $\mathrm{M}$ & Standardbred & 160.0 & 156.2 & 101.6 \\
JR & 4 & 19 & $\mathrm{G}$ & Quarter Horse & 149.9 & 149.9 & 86.4 \\
Pistol & 5 & 5 & $\mathrm{M}$ & Quarter Horse & 147.3 & 152.4 & 81.3 \\
Romeo & 6 & 10 & $\mathrm{G}$ & Quarter Horse & 170.8 & 165.1 & 96.5 \\
Susie & 7 & 18 & $\mathrm{M}$ & Quarter Horse & 151.8 & 158.8 & 95.3 \\
Sweetie & 8 & 15 & $\mathrm{M}$ & Quarter Horse & 157.5 & 165.1 & 104.1 \\
\hline
\end{tabular}

1850 


\begin{tabular}{|c|c|c|c|c|c|c|c|}
\hline Horse & Day & $\begin{array}{l}\text { Girth } \\
\text { Circ. } \\
(\mathrm{cm})\end{array}$ & $\begin{array}{l}\text { Abd. } \\
\text { Circ. } \\
(\mathrm{cm})\end{array}$ & $\begin{array}{c}\text { Neck } \\
\text { Crest } \\
\text { Height }\end{array}$ & $\begin{array}{l}\text { Neck Circ. } \\
\text { at } 0.25 \mathrm{NL} \\
\quad(\mathrm{cm})\end{array}$ & $\begin{array}{l}\text { Neck Circ. } \\
\text { at } 0.5 \mathrm{NL} \\
(\mathrm{cm})\end{array}$ & $\begin{array}{l}\text { Neck Circ. } \\
\text { at } 0.75 \mathrm{NL} \\
(\mathrm{cm})\end{array}$ \\
\hline Boots & 0 & 187.96 & 208.28 & 10.16 & 77.47 & 95.25 & 110.49 \\
\hline Boots & 14 & 189.23 & 200.66 & 10.16 & 78.40 & 88.90 & 109.22 \\
\hline Boots & 28 & 187.50 & 201.00 & 10.00 & 73.50 & 89.00 & 104.00 \\
\hline Boots & 42 & 184.00 & 195.00 & 10.00 & 73.00 & 89.00 & 105.00 \\
\hline Boots & 70 & 182.00 & 190.00 & 8.00 & 74.50 & 90.00 & 106.00 \\
\hline Boots & 98 & 179.00 & 187.00 & 7.50 & 70.00 & 90.00 & 103.00 \\
\hline Daphne & 0 & 184.15 & 196.85 & 12.07 & 72.39 & 91.66 & 106.64 \\
\hline Daphne & 14 & 189.00 & 198.00 & 12.00 & 76.00 & 90.00 & 105.00 \\
\hline Daphne & 28 & 184.00 & 191.00 & 11.50 & 73.00 & 90.00 & 112.50 \\
\hline Daphne & 42 & 189.00 & 189.00 & 11.50 & 72.00 & 91.00 & 103.00 \\
\hline Daphne & 70 & 183.00 & 190.00 & 10.00 & 78.00 & 92.00 & 107.00 \\
\hline Daphne & 98 & 183.00 & 188.00 & 9.00 & 71.00 & 85.00 & 101.00 \\
\hline Getda & 0 & 201.93 & 226.06 & 11.43 & 79.38 & 102.24 & 118.75 \\
\hline Getda & 14 & 202.00 & 217.00 & 12.00 & 77.50 & 101.40 & 117.00 \\
\hline Getda & 28 & 206.50 & 219.50 & . & 76.00 & 92.00 & 107.50 \\
\hline Getda & 42 & 202.00 & 216.50 & 9.25 & 76.00 & 87.50 & 105.00 \\
\hline Getda & 70 & 220.50 & 221.50 & 8.50 & 74.00 & 93.50 & 104.00 \\
\hline Getda & 98 & . & . & 7.00 & 72.00 & 91.50 & 102.00 \\
\hline JR & 0 & 189.23 & 215.90 & 13.97 & 79.38 & 102.87 & 123.19 \\
\hline JR & 14 & 187.00 & 213.00 & 15.24 & 78.00 & 91.00 & 113.00 \\
\hline JR & 28 & 184.00 & 208.00 & 13.00 & 80.00 & 90.50 & 116.00 \\
\hline JR & 42 & 182.00 & 286.00 & 10.00 & 78.00 & 91.00 & 110.00 \\
\hline JR & 70 & 186.00 & 199.00 & 10.00 & 78.00 & 105.00 & 119.00 \\
\hline Pistol & 0 & 186.69 & 208.28 & 10.80 & 69.85 & 91.44 & 109.22 \\
\hline Pistol & 14 & 187.00 & 196.00 & 10.00 & 83.00 & 105.00 & 113.00 \\
\hline Pistol & 28 & 189.00 & 203.00 & 9.00 & 68.50 & 93.00 & 107.00 \\
\hline Pistol & 42 & 187.00 & 200.00 & 9.00 & 76.00 & 97.00 & 107.00 \\
\hline Pistol & 70 & 179.50 & 182.50 & 10.00 & 73.50 & 85.50 & 106.00 \\
\hline Pistol & 98 & 187.50 & 196.00 & 5.50 & 76.00 & 90.50 & 106.00 \\
\hline Romeo & 0 & 212.09 & 231.14 & 12.07 & 87.00 & 106.68 & 125.73 \\
\hline Romeo & 14 & 218.00 & 230.00 & 12.00 & 89.00 & 104.50 & 125.50 \\
\hline Romeo & 28 & 210.00 & 233.00 & 11.00 & 89.00 & 104.00 & 128.00 \\
\hline Romeo & 42 & 214.50 & 225.50 & 10.50 & 85.50 & 102.00 & 124.00 \\
\hline Romeo & 70 & 208.00 & 228.00 & 9.00 & 82.00 & 98.00 & 127.00 \\
\hline Romeo & 98 & 203.00 & 225.00 & 9.50 & 82.00 & 98.00 & 125.00 \\
\hline
\end{tabular}




\begin{tabular}{cccccccc}
\hline \hline Horse & Day & $\begin{array}{c}\text { Girth } \\
\text { Circ. } \\
(\mathrm{cm})\end{array}$ & $\begin{array}{c}\text { Abd. } \\
\text { Circ. } \\
(\mathrm{cm})\end{array}$ & $\begin{array}{c}\text { Neck } \\
\text { Crest } \\
\text { Height }\end{array}$ & $\begin{array}{c}\text { Neck Circ. } \\
\text { at 0.25 NL } \\
(\mathrm{cm})\end{array}$ & $\begin{array}{c}\text { Neck Circ. } \\
\text { at 0.5 NL } \\
(\mathrm{cm})\end{array}$ & $\begin{array}{c}\text { Neck Circ. } \\
\text { at 0.75 NL } \\
(\mathrm{cm})\end{array}$ \\
\hline Susie & 0 & 194.31 & 220.98 & 12.07 & 79.38 & 102.87 & 120.02 \\
Susie & 14 & 191.77 & 224.79 & 13.97 & 76.84 & 102.87 & 120.02 \\
Susie & 28 & 196.00 & 207.00 & 10.00 & 84.00 & 94.50 & 109.00 \\
Susie & 42 & 188.00 & 213.00 &. & 76.00 & 94.00 & 103.00 \\
Susie & 70 & 188.00 & 210.00 & 11.00 & 78.00 & 103.00 & 116.00 \\
Susie & 98 & 182.50 & 208.00 & 6.50 & 75.50 & 92.50 & 113.00 \\
Sweetie & 0 & 200.66 & 213.36 & 13.97 & 85.09 & 111.13 & 129.86 \\
Sweetie & 14 & 196.85 & 217.17 & 13.97 & 81.92 & 109.22 & 129.54 \\
Sweetie & 28 & 195.50 & 205.50 & 11.50 & 80.00 & 99.50 & 114.50 \\
Sweetie & 42 & 196.00 & 207.00 & 9.00 & 82.00 & 100.00 & 121.00 \\
Sweetie & 70 & 199.00 & 199.00 & 12.00 & 78.00 & 106.00 & 132.00 \\
Sweetie & 98 & 197.00 & 195.00 & 6.00 & 77.50 & 90.00 & 108.00 \\
\hline
\end{tabular}

1853

1854 
APPENDIX C

\begin{tabular}{|c|c|c|c|c|c|c|c|c|}
\hline Horse & Day & BCS & $\mathrm{CNS}$ & $\begin{array}{c}\text { Rump Fat } \\
\text { Thickness } \\
\text { (mm) }\end{array}$ & $\begin{array}{c}\text { Percent } \\
\text { Fat }\end{array}$ & $\begin{array}{c}\text { Fat } \\
\text { Mass } \\
(\mathrm{kg})\end{array}$ & $\begin{array}{c}\text { Fat Free } \\
\text { Mass } \\
(\mathrm{kg})\end{array}$ & $\begin{array}{c}\text { Weight } \\
(\mathrm{kg})\end{array}$ \\
\hline Boots & 0 & 8 & 3 & 9 & 7.39 & 36.4 & 455.9 & 492.3 \\
\hline Boots & 14 & 8 & 3 & 8 & 6.85 & 34.9 & 474.2 & 509.1 \\
\hline Boots & 28 & 8 & 3 & 8 & 6.85 & 33.0 & 449.3 & 482.3 \\
\hline Boots & 42 & 8 & 3 & 9 & 7.39 & 35.5 & 444.1 & 479.5 \\
\hline Boots & 70 & 6.5 & 2.5 & 7.5 & 6.57 & 30.3 & 430.6 & 460.9 \\
\hline Boots & 98 & 5 & 2 & 3 & 4.11 & 18.3 & 427.1 & 445.5 \\
\hline Daphne & 0 & 8 & 3 & 19 & 12.86 & 55.6 & 376.7 & 432.3 \\
\hline Daphne & 14 & 8 & 3 & 19 & 12.86 & 55.4 & 375.1 & 430.5 \\
\hline Daphne & 28 & 8 & 3 & 18 & 12.32 & 52.9 & 376.6 & 429.5 \\
\hline Daphne & 42 & 8 & 3 & 18 & 12.32 & 52.1 & 371.1 & 423.2 \\
\hline Daphne & 70 & 7 & 2.5 & 7 & 6.30 & 29.3 & 435.3 & 464.5 \\
\hline Daphne & 98 & 5 & 2.5 & 10 & 7.94 & 32.7 & 378.7 & 411.4 \\
\hline Getda & 0 & 8 & 2.5 & 5 & 5.21 & 30.1 & 547.7 & 577.7 \\
\hline Getda & 14 & 8 & 2.5 & 10 & 7.94 & 45.5 & 527.3 & 572.7 \\
\hline Getda & 28 & 7.5 & 2.5 & 9 & 7.39 & 41.6 & 521.5 & 563.2 \\
\hline Getda & 42 & 7.5 & 2 & 6 & 5.75 & 32.0 & 524.8 & 556.8 \\
\hline Getda & 70 & 5.5 & 2 & 3 & 4.11 & 22.9 & 533.9 & 556.8 \\
\hline Getda & 98 & 5 & 2 & 3 & 4.11 & 21.9 & 511.7 & 533.6 \\
\hline JR & 0 & 8 & 2.5 & 10 & 7.94 & 42.4 & 492.1 & 534.5 \\
\hline JR & 14 & 7 & 2.5 & 8 & 6.85 & 35.5 & 482.7 & 518.2 \\
\hline JR & 28 & 6.5 & 2 & 11 & 8.49 & 42.7 & 460.1 & 502.7 \\
\hline $\mathrm{JR}$ & 42 & 6 & 2 & 8 & 6.85 & 34.0 & 463.2 & 497.3 \\
\hline JR & 70 & 5 & 2 & 4 & 4.66 & 22.4 & 457.6 & 480.0 \\
\hline Pistol & 0 & 7 & 2.5 & 9.5 & 7.67 & 37.8 & 455.0 & 492.7 \\
\hline Pistol & 14 & 7.5 & 2.5 & 10 & 7.94 & 38.4 & 445.7 & 484.1 \\
\hline Pistol & 28 & 7.5 & . & 11 & 8.49 & 41.5 & 448.0 & 489.5 \\
\hline Pistol & 42 & 7.5 & 2.5 & 8 & 8.49 & 41.3 & 445.1 & 486.4 \\
\hline Pistol & 70 & 6.5 & 2.5 & 12 & 9.03 & 37.6 & 378.3 & 415.9 \\
\hline Pistol & 98 & 5 & 2 & 6.5 & 6.03 & 28.3 & 441.7 & 470.0 \\
\hline Romeo & 0 & 8 & 2.5 & 12 & 9.03 & 62.3 & 627.3 & 689.5 \\
\hline Romeo & 14 & 8 & 2.5 & 14 & 10.13 & 70.4 & 625.0 & 695.5 \\
\hline Romeo & 28 & 7.5 & 2.5 & 8 & 6.85 & 46.1 & 627.1 & 673.2 \\
\hline Romeo & 42 & 7.5 & 2.5 & 9 & 7.39 & 49.5 & 620.5 & 670.0 \\
\hline Romeo & 70 & 5.5 & 2 & 9 & 7.39 & 45.5 & 570.0 & 615.5 \\
\hline Romeo & 98 & 5 & 2 & 6.5 & 6.03 & 39.4 & 615.1 & 654.5 \\
\hline
\end{tabular}




\begin{tabular}{ccccccccc}
\hline \hline Horse & Day & BCS & CNS & $\begin{array}{c}\text { Rump Fat } \\
\text { Thickness } \\
(\mathrm{mm})\end{array}$ & $\begin{array}{c}\text { Percent } \\
\text { Fat }\end{array}$ & $\begin{array}{c}\text { Fat } \\
\text { Mass } \\
(\mathrm{kg})\end{array}$ & $\begin{array}{c}\text { Fat Free } \\
\text { Mass } \\
(\mathrm{kg})\end{array}$ & $\begin{array}{c}\text { Weight } \\
(\mathrm{kg})\end{array}$ \\
\hline Susie & 0 & 7.5 & 2.5 & 10 & 7.94 & 43.3 & 502.1 & 545.5 \\
Susie & 14 & 7.5 & 2.5 & 10 & 7.94 & 43.0 & 498.4 & 541.4 \\
Susie & 28 & 7 & 2.5 & 9 & 7.39 & 40.0 & 500.9 & 540.9 \\
Susie & 42 & 7 & 2.5 & 8.5 & 7.12 & 38.0 & 495.6 & 533.6 \\
Susie & 70 & 6.5 & 2 & 8 & 6.85 & 36.1 & 490.8 & 526.8 \\
Susie & 98 & 5 & 2 & 10 & 7.94 & 40.6 & 470.3 & 510.9 \\
Sweetie & 0 & 8 & 3 & 14 & 10.13 & 63.1 & 559.7 & 622.7 \\
Sweetie & 14 & 8 & 2.5 & 11 & 8.49 & 52.5 & 565.7 & 618.2 \\
Sweetie & 28 & 7.5 & 3 & 9 & 7.39 & 43.8 & 548.1 & 591.8 \\
Sweetie & 42 & 7.5 & 3 & 9.5 & 7.67 & 45.5 & 548.1 & 593.6 \\
Sweetie & 70 & 6.5 & 2 & 6 & 5.75 & 32.1 & 525.6 & 557.7 \\
Sweetie & 98 & 5 & 2 & 4 & 4.66 & 22.5 & 461.1 & 483.6 \\
\hline
\end{tabular}

1857

1858 


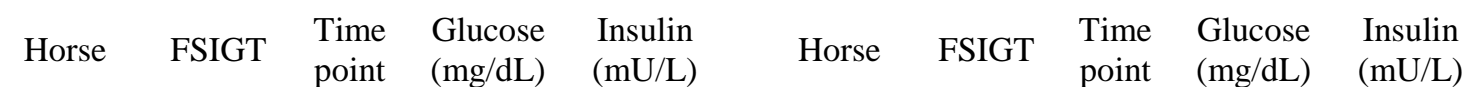

\begin{tabular}{|c|c|c|c|c|c|c|c|c|c|}
\hline Boots & 1 & -30 & 141.68 & 4.33 & Boots & 2 & 3 & 294.16 & 15.91 \\
\hline Boots & 1 & 0 & 235.75 & 14.66 & Boots & 2 & 4 & 310.73 & 12.75 \\
\hline Boots & 1 & 1 & 383.04 & . & Boots & 2 & 5 & 318.94 & 14.87 \\
\hline Boots & 1 & 2 & 316.40 & 15.15 & Boots & 2 & 6 & 317.84 & 12.64 \\
\hline Boots & 1 & 3 & 341.84 & 11.16 & Boots & 2 & 7 & 314.74 & 15.10 \\
\hline Boots & 1 & 4 & 313.55 & . & Boots & 2 & 8 & 299.42 & 12.43 \\
\hline Boots & 1 & 5 & 330.58 & 19.25 & Boots & 2 & 9 & 290.32 & 10.53 \\
\hline Boots & 1 & 6 & 350.10 & 16.97 & Boots & 2 & 10 & 287.95 & 11.61 \\
\hline Boots & 1 & 7 & 314.74 & . & Boots & 2 & 12 & 320.33 & 11.18 \\
\hline Boots & 1 & 8 & 324.18 & 13.86 & Boots & 2 & 14 & 304.45 & 11.19 \\
\hline Boots & 1 & 10 & 321.58 & 16.83 & Boots & 2 & 16 & 295.61 & 9.36 \\
\hline Boots & 1 & 12 & 279.38 & 17.67 & Boots & 2 & 19 & 281.13 & 8.54 \\
\hline Boots & 1 & 14 & 296.33 & 13.19 & Boots & 2 & 22 & 241.12 & 212.42 \\
\hline Boots & 1 & 16 & 321.90 & 14.82 & Boots & 2 & 23 & 255.45 & 157.48 \\
\hline Boots & 1 & 19 & 292.43 & 14.01 & Boots & 2 & 24 & 265.69 & 135.75 \\
\hline Boots & 1 & 22 & 318.97 & 12.20 & Boots & 2 & 25 & 265.34 & 111.98 \\
\hline Boots & 1 & 23 & 300.36 & 216.46 & Boots & 2 & 27 & 241.13 & 77.04 \\
\hline Boots & 1 & 24 & 278.48 & 209.35 & Boots & 2 & 30 & 229.18 & 64.57 \\
\hline Boots & 1 & 25 & 312.03 & 195.40 & Boots & 2 & 35 & 239.62 & 30.20 \\
\hline Boots & 1 & 27 & 270.37 & 122.02 & Boots & 2 & 40 & 179.81 & 21.09 \\
\hline Boots & 1 & 30 & 303.59 & 116.60 & Boots & 2 & 50 & 176.35 & 8.72 \\
\hline Boots & 1 & 35 & 287.61 & 70.45 & Boots & 2 & 60 & 121.16 & 5.01 \\
\hline Boots & 1 & 40 & 268.40 & . & Boots & 2 & 70 & 125.42 & 3.50 \\
\hline Boots & 1 & 50 & 276.99 & . & Boots & 2 & 80 & 110.32 & 5.55 \\
\hline Boots & 1 & 60 & 264.61 & . & Boots & 2 & 90 & 108.76 & 3.90 \\
\hline Boots & 1 & 70 & 234.09 & . & Boots & 2 & 100 & 129.76 & . \\
\hline Boots & 1 & 80 & 225.45 & . & Boots & 2 & 120 & 101.34 & 2.78 \\
\hline Boots & 1 & 90 & 244.76 & . & Boots & 2 & 150 & 109.89 & 2.43 \\
\hline Boots & 1 & 100 & 313.21 & 14.94 & Boots & 2 & 180 & 103.40 & 2.60 \\
\hline Boots & 1 & 120 & 217.32 & 15.68 & Daphne & 1 & -30 & 104.14 & 3.71 \\
\hline Boots & 1 & 150 & 195.43 & 11.31 & Daphne & 1 & 0 & 275.64 & 3.10 \\
\hline Boots & 1 & 180 & 161.08 & 5.26 & Daphne & 1 & 1 & 299.76 & 26.49 \\
\hline Boots & 1 & 240 & . & 5.17 & Daphne & 1 & 2 & 282.86 & 28.15 \\
\hline Boots & 2 & -30 & 159.89 & 4.63 & Daphne & 1 & 3 & 314.47 & 26.30 \\
\hline Boots & 2 & 0 & 136.29 & 3.90 & Daphne & 1 & 4 & 292.44 & 30.51 \\
\hline Boots & 2 & 1 & 318.75 & 63.06 & Daphne & 1 & 5 & 323.67 & 29.17 \\
\hline Boots & 2 & 2 & 315.23 & 13.18 & Daphne & 1 & 6 & 317.14 & 30.73 \\
\hline
\end{tabular}




\begin{tabular}{|c|c|c|c|c|c|c|c|c|c|}
\hline Horse & FSIGT & $\begin{array}{l}\text { Time } \\
\text { point }\end{array}$ & $\begin{array}{l}\text { Glucose } \\
(\mathrm{mg} / \mathrm{dL})\end{array}$ & $\begin{array}{l}\text { Insulin } \\
(\mathrm{mU} / \mathrm{L})\end{array}$ & Horse & FSIGT & $\begin{array}{l}\text { Time } \\
\text { point }\end{array}$ & $\begin{array}{l}\text { Glucose } \\
(\mathrm{mg} / \mathrm{dL})\end{array}$ & $\begin{array}{l}\text { Insulin } \\
(\mathrm{mU} / \mathrm{L})\end{array}$ \\
\hline Daphne & 1 & 7 & 305.82 & 31.35 & Daphne & 2 & 19 & 320.66 & 13.43 \\
\hline Daphne & 1 & 8 & 328.55 & 35.77 & Daphne & 2 & 22 & 234.75 & 319.58 \\
\hline Daphne & 1 & 10 & 289.76 & 36.57 & Daphne & 2 & 23 & 245.05 & 202.54 \\
\hline Daphne & 1 & 12 & 266.90 & . & Daphne & 2 & 24 & 277.02 & 162.85 \\
\hline Daphne & 1 & 14 & 326.60 & 29.17 & Daphne & 2 & 25 & 282.56 & 145.21 \\
\hline Daphne & 1 & 16 & 319.96 & 26.91 & Daphne & 2 & 27 & 268.37 & 109.06 \\
\hline Daphne & 1 & 19 & 279.35 & 27.65 & Daphne & 2 & 30 & 270.67 & 80.82 \\
\hline Daphne & 1 & 22 & 323.11 & 319.06 & Daphne & 2 & 35 & 272.30 & 51.44 \\
\hline Daphne & 1 & 23 & 323.41 & 276.25 & Daphne & 2 & 40 & 222.53 & 36.17 \\
\hline Daphne & 1 & 24 & 270.46 & 235.25 & Daphne & 2 & 50 & 186.83 & 25.42 \\
\hline Daphne & 1 & 25 & 312.21 & 205.59 & Daphne & 2 & 60 & 156.42 & 23.47 \\
\hline Daphne & 1 & 27 & 304.55 & 175.44 & Daphne & 2 & 70 & 148.35 & 17.48 \\
\hline Daphne & 1 & 30 & 303.59 & 143.07 & Daphne & 2 & 80 & 127.03 & 14.31 \\
\hline Daphne & 1 & 35 & 322.79 & 100.61 & Daphne & 2 & 90 & 116.93 & 12.19 \\
\hline Daphne & 1 & 40 & 275.57 & 83.83 & Daphne & 2 & 100 & 121.23 & 10.98 \\
\hline Daphne & 1 & 50 & 283.08 & 61.73 & Daphne & 2 & 120 & 111.28 & 8.60 \\
\hline Daphne & 1 & 60 & 253.30 & 56.73 & Daphne & 2 & 150 & 97.54 & 7.12 \\
\hline Daphne & 1 & 70 & 202.07 & 55.26 & Daphne & 2 & 180 & 85.73 & 5.26 \\
\hline Daphne & 1 & 80 & 192.61 & 54.56 & Getda & 1 & -30 & 138.60 & 5.33 \\
\hline Daphne & 1 & 90 & 184.95 & 56.36 & Getda & 1 & 0 & 114.40 & 7.82 \\
\hline Daphne & 1 & 100 & 211.14 & 52.16 & Getda & 1 & 1 & 344.47 & 43.95 \\
\hline Daphne & 1 & 120 & 154.28 & 37.99 & Getda & 1 & 2 & 339.88 & 49.32 \\
\hline Daphne & 1 & 150 & 103.36 & 19.97 & Getda & 1 & 3 & 285.15 & 56.80 \\
\hline Daphne & 1 & 180 & 79.34 & 10.27 & Getda & 1 & 4 & 291.63 & 58.64 \\
\hline Daphne & 1 & 240 & 78.57 & 2.54 & Getda & 1 & 5 & 344.35 & 56.79 \\
\hline Daphne & 2 & -30 & 117.99 & 6.75 & Getda & 1 & 6 & 325.73 & 50.64 \\
\hline Daphne & 2 & 0 & 110.45 & 5.80 & Getda & 1 & 7 & 307.23 & 50.23 \\
\hline Daphne & 2 & 1 & 311.43 & 18.45 & Getda & 1 & 8 & 290.62 & 55.36 \\
\hline Daphne & 2 & 2 & 330.59 & 18.26 & Getda & 1 & 9 & . & . \\
\hline Daphne & 2 & 3 & 301.42 & 16.84 & Getda & 1 & 10 & 289.01 & 53.33 \\
\hline Daphne & 2 & 4 & 340.42 & 19.77 & Getda & 1 & 12 & 299.19 & 42.29 \\
\hline Daphne & 2 & 5 & 302.35 & 22.05 & Getda & 1 & 14 & 264.01 & 45.03 \\
\hline Daphne & 2 & 6 & 299.04 & . & Getda & 1 & 16 & 260.86 & 42.39 \\
\hline Daphne & 2 & 7 & 296.82 & 19.07 & Getda & 1 & 19 & 302.16 & 45.08 \\
\hline Daphne & 2 & 8 & 308.08 & 17.79 & Getda & 1 & 22 & 266.90 & 495.47 \\
\hline Daphne & 2 & 9 & 297.40 & 17.13 & Getda & 1 & 23 & 312.61 & 290.42 \\
\hline Daphne & 2 & 10 & 296.06 & 14.90 & Getda & 1 & 24 & 283.87 & 297.12 \\
\hline Daphne & 2 & 12 & 241.63 & 16.00 & Getda & 1 & 25 & 303.60 & 226.22 \\
\hline
\end{tabular}




\begin{tabular}{|c|c|c|c|c|c|c|c|c|c|}
\hline Horse & FSIGT & $\begin{array}{l}\text { Time } \\
\text { point }\end{array}$ & $\begin{array}{l}\text { Glucose } \\
(\mathrm{mg} / \mathrm{dL})\end{array}$ & $\begin{array}{l}\text { Insulin } \\
(\mathrm{mU} / \mathrm{L})\end{array}$ & Horse & FSIGT & $\begin{array}{l}\text { Time } \\
\text { point }\end{array}$ & $\begin{array}{l}\text { Glucose } \\
(\mathrm{mg} / \mathrm{dL})\end{array}$ & $\begin{array}{l}\text { Insulin } \\
(\mathrm{mU} / \mathrm{L})\end{array}$ \\
\hline Daphne & 2 & 14 & 306.41 & 13.30 & Getda & 1 & 27 & 292.28 & 189.35 \\
\hline Daphne & 2 & 16 & 303.61 & 14.74 & Getda & 1 & 30 & 257.78 & 160.45 \\
\hline Getda & 1 & 35 & 275.64 & 107.56 & Getda & 2 & 90 & 144.64 & 34.96 \\
\hline Getda & 1 & 40 & 258.68 & 95.56 & Getda & 2 & 100 & . & 31.25 \\
\hline Getda & 1 & 50 & 289.89 & 71.01 & Getda & 2 & 120 & 174.23 & 24.20 \\
\hline Getda & 1 & 60 & 273.22 & 51.50 & Getda & 2 & 150 & 105.65 & 14.75 \\
\hline Getda & 1 & 70 & 226.50 & 47.19 & Getda & 2 & 180 & 94.60 & 8.67 \\
\hline Getda & 1 & 80 & 226.30 & 49.90 & $\mathrm{JR}$ & 1 & -30 & 108.86 & 2.10 \\
\hline Getda & 1 & 90 & 193.50 & 39.32 & $\mathrm{JR}$ & 1 & 0 & 105.99 & 2.69 \\
\hline Getda & 1 & 100 & 184.48 & 40.32 & $\mathrm{JR}$ & 1 & 1 & 297.73 & 14.39 \\
\hline Getda & 1 & 120 & 209.05 & 30.25 & $\mathrm{JR}$ & 1 & 2 & 337.42 & 19.13 \\
\hline Getda & 1 & 150 & 308.45 & 25.52 & $\mathrm{JR}$ & 1 & 3 & 302.18 & 16.34 \\
\hline Getda & 1 & 180 & 149.21 & 16.49 & $\mathrm{JR}$ & 1 & 4 & 329.24 & 18.32 \\
\hline Getda & 1 & 240 & 113.62 & 6.84 & $\mathrm{JR}$ & 1 & 5 & 303.17 & 25.48 \\
\hline Getda & 2 & -30 & 120.66 & 10.00 & $\mathrm{JR}$ & 1 & 6 & 302.69 & 27.34 \\
\hline Getda & 2 & 0 & 107.47 & 6.27 & $\mathrm{JR}$ & 1 & 7 & 295.08 & 21.44 \\
\hline Getda & 2 & 1 & 266.81 & 56.90 & $\mathrm{JR}$ & 1 & 8 & 189.98 & 26.11 \\
\hline Getda & 2 & 2 & 298.70 & 59.08 & $\mathrm{JR}$ & 1 & 10 & 299.49 & 23.60 \\
\hline Getda & 2 & 3 & 319.24 & 63.57 & $\mathrm{JR}$ & 1 & 12 & 304.89 & 22.14 \\
\hline Getda & 2 & 4 & 325.87 & 64.15 & $\mathrm{JR}$ & 1 & 14 & 274.79 & 18.00 \\
\hline Getda & 2 & 5 & 267.30 & 60.61 & $\mathrm{JR}$ & 1 & 16 & 304.23 & 16.38 \\
\hline Getda & 2 & 6 & 318.81 & 56.03 & $\mathrm{JR}$ & 1 & 19 & 313.16 & 14.86 \\
\hline Getda & 2 & 7 & 298.33 & 45.81 & $\mathrm{JR}$ & 1 & 22 & 309.72 & 13.67 \\
\hline Getda & 2 & 8 & 322.77 & 50.47 & $\mathrm{JR}$ & 1 & 23 & 295.35 & 302.64 \\
\hline Getda & 2 & 9 & 330.95 & 50.70 & $\mathrm{JR}$ & 1 & 24 & 291.86 & 247.29 \\
\hline Getda & 2 & 10 & 250.40 & 44.25 & $\mathrm{JR}$ & 1 & 25 & 288.14 & 173.85 \\
\hline Getda & 2 & 12 & 314.18 & 35.23 & $\mathrm{JR}$ & 1 & 27 & 259.12 & 155.52 \\
\hline Getda & 2 & 14 & 285.19 & 37.10 & $\mathrm{JR}$ & 1 & 30 & 230.51 & 121.99 \\
\hline Getda & 2 & 16 & 277.54 & 33.94 & $\mathrm{JR}$ & 1 & 35 & 261.32 & 91.19 \\
\hline Getda & 2 & 19 & 302.05 & 29.91 & $\mathrm{JR}$ & 1 & 40 & 237.79 & 53.21 \\
\hline Getda & 2 & 22 & 270.00 & 358.34 & $\mathrm{JR}$ & 1 & 50 & 324.91 & 34.54 \\
\hline Getda & 2 & 23 & 266.52 & 249.23 & $\mathrm{JR}$ & 1 & 60 & 168.11 & 22.71 \\
\hline Getda & 2 & 24 & 262.68 & 142.11 & $\mathrm{JR}$ & 1 & 70 & 202.77 & 18.25 \\
\hline Getda & 2 & 25 & 257.66 & 173.82 & $\mathrm{JR}$ & 1 & 80 & 167.08 & 17.39 \\
\hline Getda & 2 & 27 & 264.22 & 142.79 & $\mathrm{JR}$ & 1 & 90 & 146.75 & 14.60 \\
\hline Getda & 2 & 30 & 233.61 & 129.90 & $\mathrm{JR}$ & 1 & 100 & 157.64 & 10.97 \\
\hline Getda & 2 & 35 & 271.35 & 69.62 & $\mathrm{JR}$ & 1 & 120 & 136.10 & 9.93 \\
\hline Getda & 2 & 40 & 248.74 & 54.66 & $\mathrm{JR}$ & 1 & 150 & 124.94 & 9.18 \\
\hline
\end{tabular}




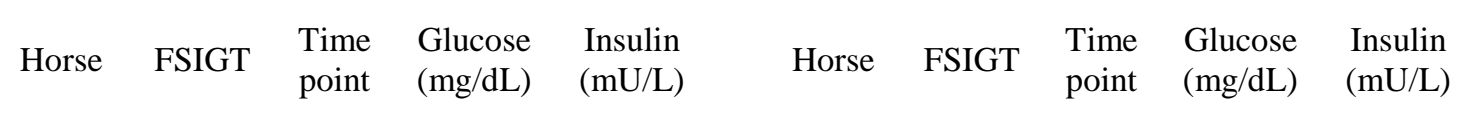

\begin{tabular}{|c|c|c|c|c|c|c|c|c|c|}
\hline Getda & 2 & 50 & 232.23 & 43.40 & $\mathrm{JR}$ & 1 & 180 & 100.26 & 4.87 \\
\hline Getda & 2 & 60 & 240.93 & 37.20 & $\mathrm{JR}$ & 1 & 240 & 287.76 & 4.46 \\
\hline Getda & 2 & 70 & 177.26 & 41.98 & $\mathrm{JR}$ & 1 & 240 & 78.04 & 1.71 \\
\hline Getda & 2 & 80 & 174.74 & 18.40 & $\mathrm{JR}$ & 2 & -30 & 148.64 & 2.42 \\
\hline $\mathrm{JR}$ & 2 & 0 & 130.27 & 2.50 & Pistol & 1 & 7 & 342.84 & 10.41 \\
\hline JR & 2 & 1 & 320.24 & . & Pistol & 1 & 8 & 313.05 & \\
\hline $\mathrm{JR}$ & 2 & 2 & 327.41 & 6.37 & Pistol & 1 & 10 & 289.89 & 11.97 \\
\hline JR & 2 & 3 & 315.76 & 8.58 & Pistol & 1 & 12 & 314.55 & 7.89 \\
\hline $\mathrm{JR}$ & 2 & 4 & 290.79 & 8.02 & Pistol & 1 & 14 & 290.28 & 9.14 \\
\hline JR & 2 & 5 & 285.37 & 25.93 & Pistol & 1 & 16 & 264.40 & 9.08 \\
\hline JR & 2 & 6 & 311.66 & 9.88 & Pistol & 1 & 19 & 299.17 & 8.19 \\
\hline $\mathrm{JR}$ & 2 & 7 & 311.00 & 9.23 & Pistol & 1 & 22 & 287.48 & 8.63 \\
\hline $\mathrm{JR}$ & 2 & 8 & 315.39 & 14.32 & Pistol & 1 & 23 & 281.68 & 395.19 \\
\hline $\mathrm{JR}$ & 2 & 9 & 269.32 & 7.45 & Pistol & 1 & 24 & 242.53 & 252.88 \\
\hline $\mathrm{JR}$ & 2 & 10 & 272.96 & 7.36 & Pistol & 1 & 25 & 268.86 & 192.34 \\
\hline $\mathrm{JR}$ & 2 & 12 & 336.17 & 5.89 & Pistol & 1 & 27 & 317.90 & 163.09 \\
\hline $\mathrm{JR}$ & 2 & 14 & 289.88 & 6.07 & Pistol & 1 & 30 & 237.00 & 119.10 \\
\hline $\mathrm{JR}$ & 2 & 16 & 291.67 & 6.45 & Pistol & 1 & 35 & 224.42 & 353.29 \\
\hline $\mathrm{JR}$ & 2 & 19 & 282.97 & 6.71 & Pistol & 1 & 40 & 183.77 & 59.27 \\
\hline $\mathrm{JR}$ & 2 & 22 & 289.91 & 321.57 & Pistol & 1 & 50 & 176.49 & 29.60 \\
\hline $\mathrm{JR}$ & 2 & 23 & 322.04 & 198.99 & Pistol & 1 & 60 & 155.34 & 21.75 \\
\hline $\mathrm{JR}$ & 2 & 24 & 262.25 & 176.21 & Pistol & 1 & 70 & 127.26 & 11.86 \\
\hline $\mathrm{JR}$ & 2 & 25 & 288.42 & 158.79 & Pistol & 1 & 80 & 113.37 & 7.75 \\
\hline $\mathrm{JR}$ & 2 & 27 & 252.68 & 217.74 & Pistol & 1 & 90 & 125.19 & 7.90 \\
\hline $\mathrm{JR}$ & 2 & 30 & 250.13 & 120.06 & Pistol & 1 & 100 & 126.05 & 5.29 \\
\hline $\mathrm{JR}$ & 2 & 35 & 220.81 & 37.16 & Pistol & 1 & 120 & 85.34 & 5.33 \\
\hline $\mathrm{JR}$ & 2 & 40 & 214.27 & 20.77 & Pistol & 1 & 150 & 65.95 & 3.71 \\
\hline $\mathrm{JR}$ & 2 & 50 & 184.37 & 50.87 & Pistol & 1 & 180 & 98.39 & 1.84 \\
\hline $\mathrm{JR}$ & 2 & 60 & 144.35 & 9.03 & Pistol & 1 & 240 & 96.58 & 2.98 \\
\hline $\mathrm{JR}$ & 2 & 70 & 119.47 & 119.93 & Pistol & 2 & -30 & 143.88 & 4.21 \\
\hline $\mathrm{JR}$ & 2 & 80 & 114.55 & 13.51 & Pistol & 2 & 0 & 124.62 & 4.40 \\
\hline $\mathrm{JR}$ & 2 & 90 & 103.97 & 3.95 & Pistol & 2 & 1 & 337.21 & 13.71 \\
\hline $\mathrm{JR}$ & 2 & 100 & 117.25 & 7.12 & Pistol & 2 & 2 & 336.26 & 12.13 \\
\hline $\mathrm{JR}$ & 2 & 120 & 97.26 & 3.08 & Pistol & 2 & 3 & 332.70 & . \\
\hline $\mathrm{JR}$ & 2 & 150 & 87.47 & 2.31 & Pistol & 2 & 4 & 329.72 & 11.67 \\
\hline $\mathrm{JR}$ & 2 & 180 & 86.44 & 2.96 & Pistol & 2 & 5 & 330.89 & 12.30 \\
\hline Pistol & 1 & -30 & 111.13 & 4.13 & Pistol & 2 & 6 & 334.39 & 9.36 \\
\hline Pistol & 1 & 0 & 299.54 & 2.27 & Pistol & 2 & 7 & 304.52 & 8.44 \\
\hline
\end{tabular}


$\begin{array}{lllllllll}\text { Horse } & \text { FSIGT } & \begin{array}{l}\text { Time } \\ \text { point }\end{array} & \begin{array}{l}\text { Glucose } \\ (\mathrm{mg} / \mathrm{dL})\end{array} & \begin{array}{l}\text { Insulin } \\ (\mathrm{mU} / \mathrm{L})\end{array} \quad \text { Horse } & \text { FSIGT } & \begin{array}{l}\text { Time } \\ \text { point }\end{array} & \begin{array}{l}\text { Glucose } \\ (\mathrm{mg} / \mathrm{dL})\end{array} & \begin{array}{c}\text { Insulin } \\ (\mathrm{mU} / \mathrm{L})\end{array}\end{array}$

\begin{tabular}{|c|c|c|c|c|c|c|c|c|c|}
\hline Pistol & 1 & 1 & 331.75 & 10.63 & Pistol & 2 & 8 & 291.72 & 11.81 \\
\hline Pistol & 1 & 2 & 286.25 & 10.49 & Pistol & 2 & 9 & 341.71 & 11.48 \\
\hline Pistol & 1 & 3 & 330.15 & 8.69 & Pistol & 2 & 10 & 318.60 & 11.85 \\
\hline Pistol & 1 & 4 & 296.46 & 9.64 & Pistol & 2 & 12 & 278.93 & . \\
\hline Pistol & 1 & 5 & 285.39 & 9.58 & Pistol & 2 & 14 & 278.68 & 12.85 \\
\hline Pistol & 1 & 6 & 292.23 & 11.08 & Pistol & 2 & 16 & 268.34 & 10.90 \\
\hline Pistol & 2 & 19 & 286.03 & 9.00 & Romeo & 1 & 50 & 282.39 & 55.03 \\
\hline Pistol & 2 & 22 & 267.53 & 361.97 & Romeo & 1 & 60 & 235.25 & 53.85 \\
\hline Pistol & 2 & 23 & 297.68 & 107.58 & Romeo & 1 & 70 & 202.24 & 51.10 \\
\hline Pistol & 2 & 24 & 276.09 & 131.03 & Romeo & 1 & 80 & 192.67 & 44.16 \\
\hline Pistol & 2 & 25 & 275.05 & 108.16 & Romeo & 1 & 90 & 186.06 & 37.31 \\
\hline Pistol & 2 & 30 & 225.25 & 65.84 & Romeo & 1 & 100 & 157.54 & 28.18 \\
\hline Pistol & 2 & 35 & 168.86 & 35.75 & Romeo & 1 & 120 & 211.14 & 31.38 \\
\hline Pistol & 2 & 40 & 140.53 & 20.30 & Romeo & 1 & 150 & 337.94 & 14.72 \\
\hline Pistol & 2 & 50 & 86.59 & 9.27 & Romeo & 1 & 180 & 255.43 & 9.05 \\
\hline Pistol & 2 & 60 & 69.00 & 6.77 & Romeo & 1 & 240 & 105.64 & 2.51 \\
\hline Pistol & 2 & 70 & 68.62 & 6.57 & Romeo & 2 & -30 & 140.70 & 5.13 \\
\hline Pistol & 2 & 80 & 57.62 & 4.32 & Romeo & 2 & 0 & 129.23 & 4.53 \\
\hline Pistol & 2 & 90 & 52.74 & 3.66 & Romeo & 2 & 1 & 281.98 & 14.31 \\
\hline Pistol & 2 & 100 & 50.60 & 2.63 & Romeo & 2 & 2 & 322.27 & 15.36 \\
\hline Pistol & 2 & 120 & 68.77 & 1.82 & Romeo & 2 & 3 & 274.44 & 16.36 \\
\hline Pistol & 2 & 150 & 87.14 & 1.60 & Romeo & 2 & 4 & 246.25 & 14.74 \\
\hline Pistol & 2 & 180 & 102.43 & 2.04 & Romeo & 2 & 5 & 302.45 & 15.45 \\
\hline Romeo & 1 & -30 & 114.31 & 5.19 & Romeo & 2 & 7 & 287.79 & 17.11 \\
\hline Romeo & 1 & 0 & 117.09 & 3.70 & Romeo & 2 & 8 & 336.73 & 15.84 \\
\hline Romeo & 1 & 1 & 348.64 & 17.50 & Romeo & 2 & 9 & 314.70 & 18.54 \\
\hline Romeo & 1 & 2 & 332.08 & 25.20 & Romeo & 2 & 10 & 307.11 & 19.37 \\
\hline Romeo & 1 & 3 & 346.00 & 23.85 & Romeo & 2 & 12 & 316.01 & 18.87 \\
\hline Romeo & 1 & 4 & 367.21 & 27.98 & Romeo & 2 & 14 & 302.65 & 15.43 \\
\hline Romeo & 1 & 5 & 311.30 & 29.71 & Romeo & 2 & 16 & 314.29 & 17.40 \\
\hline Romeo & 1 & 6 & 327.86 & 27.57 & Romeo & 2 & 19 & 320.92 & 18.92 \\
\hline Romeo & 1 & 7 & 326.15 & 24.94 & Romeo & 2 & 22 & 310.95 & . \\
\hline Romeo & 1 & 8 & 302.74 & 34.96 & Romeo & 2 & 23 & 243.62 & 186.49 \\
\hline Romeo & 1 & 10 & 292.95 & 25.51 & Romeo & 2 & 24 & 357.99 & 176.86 \\
\hline Romeo & 1 & 12 & 113.74 & 23.63 & Romeo & 2 & 25 & 308.35 & 147.98 \\
\hline Romeo & 1 & 14 & 320.17 & 20.59 & Romeo & 2 & 27 & 323.50 & 124.21 \\
\hline Romeo & 1 & 16 & 295.35 & 26.92 & Romeo & 2 & 30 & 245.06 & 93.96 \\
\hline Romeo & 1 & 19 & 117.29 & 21.39 & Romeo & 2 & 35 & 250.84 & 72.19 \\
\hline
\end{tabular}




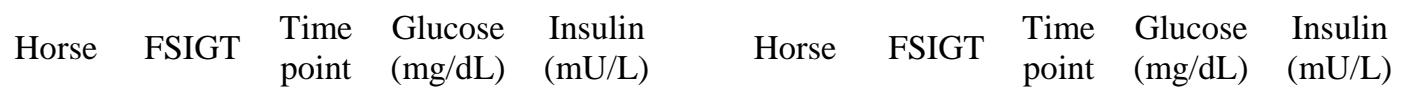

\begin{tabular}{|c|c|c|c|c|c|c|c|c|c|}
\hline Romeo & 1 & 22 & 292.27 & 522.57 & Romeo & 2 & 40 & 238.32 & 49.50 \\
\hline Romeo & 1 & 23 & 287.28 & 324.23 & Romeo & 2 & 50 & 215.14 & 36.65 \\
\hline Romeo & 1 & 24 & 321.94 & 257.02 & Romeo & 2 & 60 & 176.18 & 28.95 \\
\hline Romeo & 1 & 25 & 288.66 & 227.44 & Romeo & 2 & 70 & 166.94 & 24.43 \\
\hline Romeo & 1 & 27 & 257.60 & 160.16 & Romeo & 2 & 80 & 169.77 & 19.03 \\
\hline Romeo & 1 & 30 & 266.31 & 125.96 & Romeo & 2 & 90 & 144.03 & 15.19 \\
\hline Romeo & 1 & 35 & 247.16 & 88.73 & Romeo & 2 & 100 & 130.09 & 10.68 \\
\hline Romeo & 1 & 40 & 241.42 & 72.41 & Romeo & 2 & 120 & 111.29 & 7.76 \\
\hline Romeo & 2 & 150 & 132.52 & 5.81 & Susie & 2 & 3 & 330.13 & 47.42 \\
\hline Romeo & 2 & 180 & 106.01 & 6.97 & Susie & 2 & 4 & 290.34 & 49.33 \\
\hline Susie & 1 & -30 & 151.02 & 4.70 & Susie & 2 & 5 & 256.96 & 54.56 \\
\hline Susie & 1 & 0 & 134.90 & 3.37 & Susie & 2 & 6 & 291.88 & 60.87 \\
\hline Susie & 1 & 1 & 345.60 & 34.23 & Susie & 2 & 7 & 293.56 & 60.34 \\
\hline Susie & 1 & 2 & 327.47 & 38.59 & Susie & 2 & 8 & . & . \\
\hline Susie & 1 & 3 & 378.50 & 41.22 & Susie & 2 & 9 & 282.61 & 49.29 \\
\hline Susie & 1 & 4 & 329.47 & 43.46 & Susie & 2 & 10 & 266.52 & 66.02 \\
\hline Susie & 1 & 5 & 289.63 & 43.47 & Susie & 2 & 12 & 285.17 & 515.54 \\
\hline Susie & 1 & 6 & 296.18 & 44.92 & Susie & 2 & 14 & 240.66 & 65.98 \\
\hline Susie & 1 & 7 & 286.78 & 39.93 & Susie & 2 & 16 & 263.02 & 63.36 \\
\hline Susie & 1 & 8 & 303.82 & 44.90 & Susie & 2 & 19 & 224.48 & 73.58 \\
\hline Susie & 1 & 9 & . & . & Susie & 2 & 22 & . & · \\
\hline Susie & 1 & 10 & 311.66 & 40.50 & Susie & 2 & 23 & 198.83 & 246.49 \\
\hline Susie & 1 & 12 & 306.46 & 40.63 & Susie & 2 & 24 & 200.73 & 245.68 \\
\hline Susie & 1 & 14 & 278.22 & 35.57 & Susie & 2 & 25 & 172.00 & 204.87 \\
\hline Susie & 1 & 16 & 162.34 & 31.48 & Susie & 2 & 27 & 152.99 & 193.09 \\
\hline Susie & 1 & 19 & 269.96 & 30.29 & Susie & 2 & 30 & 110.91 & 151.79 \\
\hline Susie & 1 & 22 & 255.74 & 544.49 & Susie & 2 & 35 & 107.01 & 94.55 \\
\hline Susie & 1 & 23 & . & . & Susie & 2 & 40 & 89.59 & 56.07 \\
\hline Susie & 1 & 24 & . & . & Susie & 2 & 50 & . & 15.17 \\
\hline Susie & 1 & 25 & 300.71 & 258.13 & Susie & 2 & 60 & . & 10.21 \\
\hline Susie & 1 & 27 & 277.39 & 201.34 & Susie & 2 & 70 & . & 6.78 \\
\hline Susie & 1 & 30 & 268.37 & . & Susie & 2 & 80 & . & 3.33 \\
\hline Susie & 1 & 35 & 247.17 & 119.91 & Susie & 2 & 90 & . & 3.08 \\
\hline Susie & 1 & 40 & . & . & Susie & 2 & 100 & . & 2.35 \\
\hline Susie & 1 & 50 & 202.73 & 69.90 & Susie & 2 & 120 & 78.74 & 1.98 \\
\hline Susie & 1 & 60 & 201.88 & 43.40 & Susie & 2 & 150 & 92.35 & 3.17 \\
\hline Susie & 1 & 70 & 157.00 & 46.50 & Susie & 2 & 180 & 112.73 & 2.24 \\
\hline Susie & 1 & 80 & 145.49 & 43.93 & Sweetie & 1 & -30 & 135.95 & 6.68 \\
\hline
\end{tabular}




\begin{tabular}{|c|c|c|c|c|c|c|c|c|c|}
\hline Horse & FSIGT & $\begin{array}{l}\text { Time } \\
\text { point }\end{array}$ & $\begin{array}{l}\text { Glucose } \\
\text { (mg/dL) }\end{array}$ & $\begin{array}{l}\text { Insulin } \\
(\mathrm{mU} / \mathrm{L})\end{array}$ & Horse & FSIGT & $\begin{array}{l}\text { Time } \\
\text { point }\end{array}$ & $\begin{array}{l}\text { Glucose } \\
\text { (mg/dL) }\end{array}$ & $\begin{array}{l}\text { Insulin } \\
(\mathrm{mU} / \mathrm{L})\end{array}$ \\
\hline Susie & 1 & 90 & 154.40 & 32.30 & Sweetie & 1 & 0 & 136.04 & 7.67 \\
\hline Susie & 1 & 100 & 141.79 & 26.92 & Sweetie & 1 & 1 & 334.92 & 58.87 \\
\hline Susie & 1 & 120 & 114.86 & 21.93 & Sweetie & 1 & 2 & 332.40 & 62.43 \\
\hline Susie & 1 & 150 & . & . & Sweetie & 1 & 3 & 305.52 & 60.07 \\
\hline Susie & 1 & 180 & . & 12.74 & Sweetie & 1 & 4 & 311.66 & 60.22 \\
\hline Susie & 1 & 240 & 74.85 & 2.49 & Sweetie & 1 & 5 & 302.00 & 62.74 \\
\hline Susie & 2 & -30 & 113.92 & 5.47 & Sweetie & 1 & 6 & 344.73 & 57.02 \\
\hline Susie & 2 & 0 & 106.18 & 4.68 & Sweetie & 1 & 7 & 310.00 & 66.02 \\
\hline Susie & 2 & 1 & 307.40 & 43.35 & Sweetie & 1 & 8 & 337.66 & 50.02 \\
\hline Susie & 2 & 2 & 311.16 & 45.94 & Sweetie & 1 & 9 & . & . \\
\hline Sweetie & 1 & 10 & 309.75 & 46.88 & Sweetie & 2 & 4 & 347.50 & 23.53 \\
\hline Sweetie & 1 & 12 & 298.57 & 44.56 & Sweetie & 2 & 5 & 313.90 & . \\
\hline Sweetie & 1 & 14 & 287.51 & 37.31 & Sweetie & 2 & 6 & 336.69 & 19.10 \\
\hline Sweetie & 1 & 16 & 321.13 & 45.16 & Sweetie & 2 & 7 & 326.22 & 20.44 \\
\hline Sweetie & 1 & 19 & 286.93 & . & Sweetie & 2 & 8 & 268.01 & 21.20 \\
\hline Sweetie & 1 & 22 & 290.88 & 527.60 & Sweetie & 2 & 9 & 335.11 & 22.79 \\
\hline Sweetie & 1 & 23 & 252.36 & 400.93 & Sweetie & 2 & 10 & 297.31 & 20.20 \\
\hline Sweetie & 1 & 24 & 298.98 & 313.89 & Sweetie & 2 & 12 & 311.83 & 16.68 \\
\hline Sweetie & 1 & 25 & 270.15 & 255.29 & Sweetie & 2 & 14 & 287.89 & 17.06 \\
\hline Sweetie & 1 & 27 & 293.41 & 242.63 & Sweetie & 2 & 16 & 293.32 & . \\
\hline Sweetie & 1 & 30 & 250.15 & 193.69 & Sweetie & 2 & 19 & 321.14 & 18.09 \\
\hline Sweetie & 1 & 35 & 302.59 & 140.69 & Sweetie & 2 & 22 & 320.03 & 206.32 \\
\hline Sweetie & 1 & 40 & 277.03 & 115.78 & Sweetie & 2 & 23 & 247.99 & 194.44 \\
\hline Sweetie & 1 & 50 & 242.02 & 99.41 & Sweetie & 2 & 24 & 233.56 & 99.58 \\
\hline Sweetie & 1 & 60 & 252.28 & 65.98 & Sweetie & 2 & 25 & 259.53 & 143.98 \\
\hline Sweetie & 1 & 70 & 247.55 & 68.87 & Sweetie & 2 & 27 & 284.15 & 107.95 \\
\hline Sweetie & 1 & 80 & 261.34 & 69.03 & Sweetie & 2 & 30 & 282.03 & 89.04 \\
\hline Sweetie & 1 & 90 & 233.25 & 53.76 & Sweetie & 2 & 24 & 233.56 & 99.58 \\
\hline Sweetie & 1 & 100 & 176.97 & 53.63 & Sweetie & 2 & 25 & 259.53 & 143.98 \\
\hline Sweetie & 1 & 120 & 230.25 & 62.01 & Sweetie & 2 & 27 & 284.15 & 107.95 \\
\hline Sweetie & 1 & 150 & 144.10 & 33.95 & Sweetie & 2 & 30 & 282.03 & 89.04 \\
\hline Sweetie & 1 & 180 & 134.94 & 20.29 & Sweetie & 2 & 35 & 231.00 & 53.53 \\
\hline Sweetie & 1 & 240 & 114.65 & 65.82 & Sweetie & 2 & 40 & 227.79 & 42.53 \\
\hline Sweetie & 2 & -30 & 159.92 & 5.26 & Sweetie & 2 & 50 & 199.06 & 26.83 \\
\hline Sweetie & 2 & 0 & 133.16 & 6.05 & Sweetie & 2 & 60 & 173.27 & 20.51 \\
\hline Sweetie & 2 & 1 & 360.98 & 27.47 & Sweetie & 2 & 70 & 160.61 & 3.07 \\
\hline Sweetie & 2 & 2 & 332.12 & 21.87 & Sweetie & 2 & 80 & 146.96 & 9.83 \\
\hline Sweetie & 2 & 3 & 266.49 & 224.58 & Sweetie & 2 & 90 & 153.03 & 16.37 \\
\hline
\end{tabular}




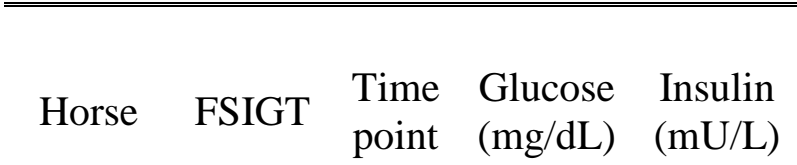

\begin{tabular}{lllll}
\hline Sweetie & 2 & 100 & 144.65 & 13.23 \\
Sweetie & 2 & 120 & 116.26 & 12.02 \\
Sweetie & 2 & 150 & 117.14 & 6.55 \\
Sweetie & 2 & 180 & 119.19 & 3.14 \\
\hline
\end{tabular}

1868

1869 
APPENDIX E

\begin{tabular}{|c|c|c|c|c|c|}
\hline Horse & Day & $\begin{array}{c}\text { Percent } \\
\text { Weight } \\
\text { Loss }\end{array}$ & $\begin{array}{l}\text { Change } \\
\text { in BCS }\end{array}$ & $\begin{array}{c}\text { Lipolysis } \\
\text { Media } \\
\text { NEFA } \\
(\mathrm{mg} / \mathrm{mL})\end{array}$ & $\begin{array}{c}\text { Lipolysis } \\
\text { Media FG } \\
(\mathrm{mg} / \mathrm{mL})\end{array}$ \\
\hline Boots & 0 & 3.30 & 0 & 0.790 & 0.021 \\
\hline Boots & 14 & 0.00 & 0 & 0.720 & 0.006 \\
\hline Boots & 28 & 5.27 & 0 & 0.782 & 0.005 \\
\hline Boots & 42 & 5.80 & 0 & 0.730 & 0.008 \\
\hline Boots & 70 & & -1.5 & 0.796 & 0.014 \\
\hline Boots & 98 & 12.50 & -3 & 0.701 & 0.010 \\
\hline Daphne & 0 & -0.42 & 0 & . & . \\
\hline Daphne & 14 & 0.00 & 0 & 1.291 & 0.029 \\
\hline Daphne & 28 & 0.21 & 0 & 0.693 & 0.007 \\
\hline Daphne & 42 & 1.69 & 0 & 0.755 & 0.009 \\
\hline Daphne & 70 & & -1 & 0.630 & 0.014 \\
\hline Daphne & 98 & 4.44 & -3 & 0.676 & 0.008 \\
\hline Getda & 0 & -0.87 & 0 & 0.884 & 0.009 \\
\hline Getda & 14 & 0.00 & 0 & 0.823 & 0.029 \\
\hline Getda & 28 & 1.67 & -0.5 & 0.764 & 0.031 \\
\hline Getda & 42 & 2.78 & -0.5 & 0.700 & 0.007 \\
\hline Getda & 70 & & -2.5 & 0.641 & 0.009 \\
\hline Getda & 98 & 6.83 & -3 & 0.586 & 0.011 \\
\hline JR & 0 & -3.16 & 1 & 0.672 & 0.032 \\
\hline $\mathrm{JR}$ & 14 & 0.00 & 0 & 0.813 & 0.017 \\
\hline $\mathrm{JR}$ & 28 & 2.98 & -0.5 & 0.626 & 0.005 \\
\hline $\mathrm{JR}$ & 42 & 4.04 & -1 & 0.575 & 0.015 \\
\hline $\mathrm{JR}$ & 70 & 7.37 & -2 & 0.714 & 0.006 \\
\hline Pistol & 0 & -1.78 & -0.5 & 0.738 & 0.007 \\
\hline Pistol & 14 & 0.00 & 0 & 0.816 & 0.012 \\
\hline Pistol & 28 & -1.13 & 0 & 0.762 & 0.020 \\
\hline Pistol & 42 & -0.47 & 0 & 0.805 & 0.005 \\
\hline Pistol & 70 & & -1 & 0.606 & 0.011 \\
\hline Pistol & 98 & 2.91 & -2.5 & 0.651 & 0.015 \\
\hline Romeo & 0 & 0.85 & 0 & 0.976 & 0.017 \\
\hline Romeo & 14 & 0.00 & 0 & 0.732 & 0.013 \\
\hline Romeo & 28 & 3.20 & -0.5 & 0.981 & 0.017 \\
\hline Romeo & 42 & 3.66 & -0.5 & 0.829 & 0.010 \\
\hline Romeo & 70 & & -2.5 & 0.852 & 0.011 \\
\hline Romeo & 98 & 5.88 & -3 & 0.735 & 0.007 \\
\hline
\end{tabular}

1871

1872 


\begin{tabular}{cccccc}
\hline \hline Horse & Day & $\begin{array}{c}\text { Percent } \\
\text { Weight } \\
\text { Loss }\end{array}$ & $\begin{array}{c}\text { Change } \\
\text { in BCS }\end{array}$ & $\begin{array}{c}\text { Lipolysis } \\
\text { Media } \\
\text { NEFA } \\
(\mathrm{mg} / \mathrm{mL})\end{array}$ & $\begin{array}{c}\text { Lipolysis } \\
\text { Media FG } \\
(\mathrm{mg} / \mathrm{mL})\end{array}$ \\
\hline Susie & 0 & -0.76 & 0 &. &. \\
Susie & 14 & 0.00 & 0 & 0.884 & 0.009 \\
Susie & 28 & 0.08 & -0.5 & 0.677 & 0.008 \\
Susie & 42 & 1.43 & -0.5 & 0.537 & 0.009 \\
Susie & 70 & & -1 &. &. \\
Susie & 98 & 5.63 & -2.5 & 0.640 & 0.017 \\
Sweetie & 0 & -0.74 & 0 & 1.171 & 0.033 \\
Sweetie & 14 & 0.00 & 0 & 0.762 & 0.007 \\
Sweetie & 28 & 4.26 & -0.5 & 0.666 & 0.007 \\
Sweetie & 42 & 3.97 & -0.5 & 0.657 & 0.012 \\
Sweetie & 70 & & -1.5 & 0.751 & 0.013 \\
Sweetie & 98 & 21.76 & -3 & 0.703 & 0.010 \\
\hline
\end{tabular}

1873

1874 
APPENDIX F

\begin{tabular}{|c|c|c|c|c|c|}
\hline Horse & Day & Subpopulation & Complex I & $\begin{array}{c}\text { Complex } \\
\text { III } \\
\end{array}$ & $\begin{array}{c}\text { Complex } \\
\text { IV }\end{array}$ \\
\hline Boots & 0 & IFM & 67.80 & 443.94 & 183.49 \\
\hline Boots & 14 & IFM & 57.13 & 280.52 & 245.19 \\
\hline Boots & 28 & IFM & 77.62 & 59.29 & 145.69 \\
\hline Boots & 42 & IFM & 56.90 & 58.21 & 139.71 \\
\hline Boots & 70 & IFM & 56.86 & 62.05 & 68.25 \\
\hline Boots & 98 & IFM & 102.00 & 55.65 & 84.59 \\
\hline Boots & 0 & SSM & 19.40 & 60.47 & 34.47 \\
\hline Boots & 14 & SSM & 16.63 & 136.14 & 71.88 \\
\hline Boots & 28 & SSM & 21.38 & 69.98 & 74.18 \\
\hline Boots & 42 & SSM & 28.85 & 23.62 & 94.46 \\
\hline Boots & 70 & SSM & 43.31 & 15.19 & 44.56 \\
\hline Boots & 98 & SSM & 37.05 & 15.16 & 87.93 \\
\hline Daphne & 0 & IFM & 37.00 & 226.44 & 72.67 \\
\hline Daphne & 14 & IFM & 31.98 & 73.28 & 113.06 \\
\hline Daphne & 28 & IFM & 74.34 & 89.66 & 117.84 \\
\hline Daphne & 42 & IFM & 48.52 & 49.64 & 115.15 \\
\hline Daphne & 70 & IFM & 31.95 & 12.07 & 94.95 \\
\hline Daphne & 98 & IFM & 42.70 & 25.41 & 58.45 \\
\hline Daphne & 0 & SSM & 68.57 & 67.82 & 52.38 \\
\hline Daphne & 14 & SSM & . & . & . \\
\hline Daphne & 28 & SSM & 46.06 & 99.78 & 47.01 \\
\hline Daphne & 42 & SSM & 45.37 & 49.51 & 77.24 \\
\hline Daphne & 70 & SSM & 49.92 & 9.90 & 61.41 \\
\hline Daphne & 98 & SSM & 20.24 & 11.05 & 50.37 \\
\hline Getda & 0 & IFM & 62.34 & 209.73 & 151.92 \\
\hline Getda & 14 & IFM & 72.31 & 114.28 & 102.86 \\
\hline Getda & 28 & IFM & 102.55 & 95.92 & 206.22 \\
\hline Getda & 42 & IFM & 18.52 & 20.21 & 53.91 \\
\hline Getda & 70 & IFM & 22.05 & 31.59 & 108.30 \\
\hline Getda & 98 & IFM & 36.95 & 36.29 & 58.06 \\
\hline Getda & 0 & SSM & 83.09 & 149.61 & 52.59 \\
\hline Getda & 14 & SSM & 34.57 & 78.34 & 41.78 \\
\hline Getda & 28 & SSM & 49.56 & 117.18 & 72.11 \\
\hline Getda & 42 & SSM & 58.66 & 10.67 & 64.01 \\
\hline Getda & 70 & SSM & 39.34 & 18.94 & 84.85 \\
\hline Getda & 98 & SSM & 19.58 & 11.45 & 50.36 \\
\hline
\end{tabular}

1876

1877

1878 


\begin{tabular}{|c|c|c|c|c|c|}
\hline Horse & Day & Subpopulation & Complex I & $\begin{array}{c}\text { Complex } \\
\text { III }\end{array}$ & $\begin{array}{c}\text { Complex } \\
\text { IV }\end{array}$ \\
\hline JR & 0 & IFM & 54.15 & 166.18 & 163.97 \\
\hline JR & 14 & IFM & 88.62 & 99.25 & 61.08 \\
\hline $\mathrm{JR}$ & 28 & IFM & 49.95 & 81.76 & 110.37 \\
\hline JR & 42 & IFM & 44.45 & 62.36 & 62.36 \\
\hline JR & 70 & IFM & 49.82 & 58.25 & 65.24 \\
\hline JR & 0 & SSM & 47.52 & 77.78 & 68.15 \\
\hline $\mathrm{JR}$ & 14 & SSM & 32.85 & 132.35 & 92.64 \\
\hline JR & 28 & SSM & 55.48 & 45.41 & 60.08 \\
\hline JR & 42 & SSM & 50.58 & 34.50 & 115.91 \\
\hline JR & 70 & SSM & 55.62 & 32.13 & 74.97 \\
\hline Pistol & 0 & IFM & 31.90 & 174.07 & 85.87 \\
\hline Pistol & 14 & IFM & 167.61 & 108.87 & 83.61 \\
\hline Pistol & 28 & IFM & 83.45 & 150.25 & 234.94 \\
\hline Pistol & 42 & IFM & 54.19 & 40.32 & 129.02 \\
\hline Pistol & 70 & IFM & 34.57 & 21.22 & 101.85 \\
\hline Pistol & 98 & IFM & 50.58 & 68.99 & 137.98 \\
\hline Pistol & 0 & SSM & 45.12 & 45.22 & 22.31 \\
\hline Pistol & 14 & SSM & 27.09 & 147.81 & 65.04 \\
\hline Pistol & 28 & SSM & 39.20 & 133.66 & 72.71 \\
\hline Pistol & 42 & SSM & 17.85 & 8.35 & 18.37 \\
\hline Pistol & 70 & SSM & 14.17 & 11.60 & 57.98 \\
\hline Pistol & 98 & SSM & 21.72 & 16.16 & 53.01 \\
\hline Romeo & 0 & IFM & 41.07 & 82.16 & 137.43 \\
\hline Romeo & 14 & IFM & 78.85 & 109.71 & 144.55 \\
\hline Romeo & 28 & IFM & . & . & 159.95 \\
\hline Romeo & 42 & IFM & 37.15 & 23.39 & 76.71 \\
\hline Romeo & 70 & IFM & . & 20.64 & 80.49 \\
\hline Romeo & 98 & IFM & 33.08 & 23.20 & 4.64 \\
\hline Romeo & 0 & SSM & 37.80 & 45.21 & 39.02 \\
\hline Romeo & 14 & SSM & 39.74 & 140.12 & 104.09 \\
\hline Romeo & 28 & SSM & 44.51 & . & 60.93 \\
\hline Romeo & 42 & SSM & 19.95 & 14.51 & 56.59 \\
\hline Romeo & 70 & SSM & 44.66 & 33.74 & 94.48 \\
\hline Romeo & 98 & SSM & 38.76 & 23.79 & 65.02 \\
\hline Susie & 0 & IFM & 18.53 & 64.46 & 62.18 \\
\hline Susie & 14 & IFM & 43.58 & 103.76 & 103.76 \\
\hline Susie & 28 & IFM & . & . & 155.22 \\
\hline Susie & 42 & IFM & 37.20 & 22.14 & 64.21 \\
\hline
\end{tabular}

1879

1880 


\begin{tabular}{cccccc}
\hline \hline Horse & Day & Subpopulation & Complex I & $\begin{array}{c}\text { Complex } \\
\text { III }\end{array}$ & $\begin{array}{c}\text { Complex } \\
\text { IV }\end{array}$ \\
\hline Susie & 70 & IFM & 34.64 & 37.81 & 109.64 \\
Susie & 98 & IFM & 28.54 & 26.69 & 26.69 \\
Susie & 0 & SSM & 28.05 & 52.48 & 85.28 \\
Susie & 14 & SSM & 55.61 & 75.86 & 64.73 \\
Susie & 28 & SSM & 40.96 & 52.15 & 137.07 \\
Susie & 42 & SSM & 42.08 & 9.84 & 58.06 \\
Susie & 70 & SSM & 28.48 & 19.98 & 53.28 \\
Susie & 98 & SSM & 23.18 & 12.65 & 40.48 \\
Sweetie & 0 & IFM & 26.15 & 61.82 & 70.38 \\
Sweetie & 14 & IFM & 65.68 & 107.51 & 225.78 \\
Sweetie & 28 & IFM & 42.84 & 105.18 & 154.26 \\
Sweetie & 42 & IFM & 73.28 & 66.64 & 175.93 \\
Sweetie & 70 & IFM & 64.51 & 44.00 & 144.31 \\
Sweetie & 98 & IFM & 18.48 & 45.38 &. \\
Sweetie & 0 & SSM & 26.54 & 100.48 & 67.35 \\
Sweetie & 14 & SSM & 37.74 & 103.90 & 69.64 \\
Sweetie & 28 & SSM & 19.70 & 89.32 & 60.54 \\
Sweetie & 42 & SSM & 28.90 & 47.31 & 87.52 \\
Sweetie & 70 & SSM & 32.68 & 35.66 & 87.97 \\
Sweetie & 98 & SSM & 9.43 & 15.44 & 30.88 \\
\hline
\end{tabular}

1881

1882 


\begin{tabular}{|c|c|c|c|c|c|}
\hline Horse & Day & Subpopulation & $\begin{array}{c}\text { ATP } \\
\text { Synthase }\end{array}$ & $\begin{array}{c}\text { Citrate } \\
\text { Synthase }\end{array}$ & $\begin{array}{c}\text { Lipid } \\
\text { Peroxidation }\end{array}$ \\
\hline Boots & 0 & IFM & 1.406 & 113.12 & 0.1527 \\
\hline Boots & 14 & IFM & 3.948 & 63.68 & 0.3232 \\
\hline Boots & 28 & IFM & 2.414 & 76.84 & 0.1750 \\
\hline Boots & 42 & IFM & . & 47.99 & 0.1868 \\
\hline Boots & 70 & IFM & 4.421 & 19.84 & 0.1073 \\
\hline Boots & 98 & IFM & 2.115 & 51.23 & 0.1578 \\
\hline Boots & 0 & SSM & 2.442 & 306.17 & . \\
\hline Boots & 14 & SSM & 2.328 & 282.26 & 0.4316 \\
\hline Boots & 28 & SSM & 1.994 & 325.64 & . \\
\hline Boots & 42 & SSM & 1.683 & 154.15 & 0.3476 \\
\hline Boots & 70 & SSM & 1.684 & 220.59 & 0.4313 \\
\hline Boots & 98 & SSM & 2.881 & 294.64 & 0.3793 \\
\hline Daphne & 0 & IFM & 2.501 & 238.78 & 0.7276 \\
\hline Daphne & 14 & IFM & 1.492 & 97.84 & 0.7900 \\
\hline Daphne & 28 & IFM & 1.825 & 110.57 & 0.3792 \\
\hline Daphne & 42 & IFM & 1.886 & 33.86 & 0.2320 \\
\hline Daphne & 70 & IFM & 0.764 & 265.58 & 0.4550 \\
\hline Daphne & 98 & IFM & 1.207 & 94.69 & 0.3296 \\
\hline Daphne & 0 & SSM & 1.111 & 273.64 & 0.6772 \\
\hline Daphne & 14 & SSM & . & . & . \\
\hline Daphne & 28 & SSM & 1.264 & 383.69 & 0.4165 \\
\hline Daphne & 42 & SSM & 0.941 & 141.09 & 0.4906 \\
\hline Daphne & 70 & SSM & 2.117 & 408.31 & 0.9560 \\
\hline Daphne & 98 & SSM & 1.259 & 378.65 & . \\
\hline Getda & 0 & IFM & 1.616 & 151.49 & 0.8532 \\
\hline Getda & 14 & IFM & 2.714 & 186.71 & 0.7077 \\
\hline Getda & 28 & IFM & 2.278 & 87.91 & 0.6325 \\
\hline Getda & 42 & IFM & 0.960 & 129.68 & 0.2622 \\
\hline Getda & 70 & IFM & 1.286 & 172.08 & 0.2638 \\
\hline Getda & 98 & IFM & 1.724 & 143.35 & . \\
\hline Getda & 0 & SSM & 1.938 & 529.52 & 0.5126 \\
\hline Getda & 14 & SSM & 1.240 & 503.41 & 0.3804 \\
\hline Getda & 28 & SSM & 2.997 & 225.83 & 0.8267 \\
\hline Getda & 42 & SSM & 1.520 & 373.12 & 0.5553 \\
\hline Getda & 70 & SSM & 2.159 & 235.05 & 0.5131 \\
\hline Getda & 98 & SSM & 2.175 & 531.35 & 0.7145 \\
\hline
\end{tabular}

1883

1884

1885

1886 


\begin{tabular}{|c|c|c|c|c|c|}
\hline Horse & Day & Subpopulation & $\begin{array}{c}\text { ATP } \\
\text { Synthase }\end{array}$ & $\begin{array}{c}\text { Citrate } \\
\text { Synthase }\end{array}$ & $\begin{array}{c}\text { Lipid } \\
\text { Peroxidation }\end{array}$ \\
\hline JR & 0 & IFM & 2.105 & 49.45 & . \\
\hline JR & 14 & IFM & 2.176 & 35.91 & 0.2424 \\
\hline $\mathrm{JR}$ & 28 & IFM & 0.971 & 30.30 & 0.1102 \\
\hline $\mathrm{JR}$ & 42 & IFM & 4.937 & 21.69 & 0.1907 \\
\hline JR & 70 & IFM & 1.107 & 35.89 & 0.0683 \\
\hline JR & 0 & SSM & 1.407 & 150.90 & 0.6942 \\
\hline JR & 14 & SSM & 2.357 & 187.61 & 0.5711 \\
\hline JR & 28 & SSM & 3.982 & 185.10 & 0.3491 \\
\hline JR & 42 & SSM & 1.311 & 99.81 & 0.2718 \\
\hline $\mathrm{JR}$ & 70 & SSM & 2.544 & 128.76 & 0.2675 \\
\hline Pistol & 0 & IFM & 1.654 & 36.95 & 0.0949 \\
\hline Pistol & 14 & IFM & 4.551 & 64.51 & 0.0967 \\
\hline Pistol & 28 & IFM & 2.595 & 52.89 & 0.1442 \\
\hline Pistol & 42 & IFM & 1.532 & 60.13 & 0.3293 \\
\hline Pistol & 70 & IFM & 0.672 & 46.33 & 0.0459 \\
\hline Pistol & 98 & IFM & 3.933 & 37.44 & 0.0821 \\
\hline Pistol & 0 & SSM & 2.291 & 243.48 & 0.8462 \\
\hline Pistol & 14 & SSM & 2.808 & 264.03 & 0.4042 \\
\hline Pistol & 28 & SSM & 4.571 & 173.59 & 0.2529 \\
\hline Pistol & 42 & SSM & 1.454 & 230.30 & 0.5857 \\
\hline Pistol & 70 & SSM & 1.377 & 168.69 & 0.3518 \\
\hline Pistol & 98 & SSM & 0.921 & 263.28 & 0.2470 \\
\hline Romeo & 0 & IFM & 2.129 & 66.75 & . \\
\hline Romeo & 14 & IFM & 8.583 & 93.45 & 0.5432 \\
\hline Romeo & 28 & IFM & 2.235 & 85.66 & 0.3866 \\
\hline Romeo & 42 & IFM & 0.889 & 89.45 & 0.4396 \\
\hline Romeo & 70 & IFM & 0.980 & 137.63 & 0.1774 \\
\hline Romeo & 98 & IFM & 2.572 & 85.41 & 0.3240 \\
\hline Romeo & 0 & SSM & 2.939 & 267.18 & . \\
\hline Romeo & 14 & SSM & 4.754 & 229.50 & 0.1669 \\
\hline Romeo & 28 & SSM & 2.831 & 183.31 & 0.4122 \\
\hline Romeo & 42 & SSM & 3.446 & 221.28 & 0.4324 \\
\hline Romeo & 70 & SSM & 3.205 & 219.82 & 0.3687 \\
\hline Romeo & 98 & SSM & 1.130 & 292.59 & 0.3497 \\
\hline Susie & 0 & IFM & 1.081 & 89.38 & 0.3483 \\
\hline Susie & 14 & IFM & 1.232 & 61.69 & 0.2953 \\
\hline Susie & 28 & IFM & 2.633 & 40.14 & 0.3365 \\
\hline Susie & 42 & IFM & 1.578 & 89.71 & 0.1587 \\
\hline
\end{tabular}




\begin{tabular}{cccccc}
\hline \hline Horse & Day & Subpopulation & $\begin{array}{c}\text { ATP } \\
\text { Synthase }\end{array}$ & $\begin{array}{c}\text { Citrate } \\
\text { Synthase }\end{array}$ & $\begin{array}{c}\text { Lipid } \\
\text { Peroxidation }\end{array}$ \\
\hline Susie & 70 & IFM & 1.796 & 61.81 & 0.3624 \\
Susie & 98 & IFM & 1.268 & 86.16 & 0.2618 \\
Susie & 0 & SSM & 1.870 & 195.80 & 0.5381 \\
Susie & 14 & SSM & 1.441 & 334.50 & 0.4622 \\
Susie & 28 & SSM & 2.123 & 122.79 & 0.3825 \\
Susie & 42 & SSM & 1.870 & 373.73 & 0.3802 \\
Susie & 70 & SSM & 1.582 & 297.03 &. \\
Susie & 98 & SSM & 1.803 & 300.94 & 0.4682 \\
Sweetie & 0 & IFM & 3.162 & 31.57 & 0.5239 \\
Sweetie & 14 & IFM & 3.830 & 50.87 & 0.2193 \\
Sweetie & 28 & IFM & 2.498 & 63.08 & 0.4065 \\
Sweetie & 42 & IFM & 1.266 & 42.02 & 0.1979 \\
Sweetie & 70 & IFM & 1.672 & 53.64 & 0.1215 \\
Sweetie & 98 & IFM & 1.437 & 40.63 & 0.0817 \\
Sweetie & 0 & SSM & 1.548 & 254.97 & 0.7668 \\
Sweetie & 14 & SSM & 1.067 & 298.52 & 0.4283 \\
Sweetie & 28 & SSM & 0.943 & 269.80 & 0.2072 \\
Sweetie & 42 & SSM & 1.685 & 160.82 & 0.2615 \\
Sweetie & 70 & SSM & 4.517 & 162.81 &. \\
Sweetie & 98 & SSM & 2.054 & 136.21 & 0.4772 \\
\hline
\end{tabular}

